\title{
Geometric Realization of Conformal Field Theory on Riemann Surfaces
}

\author{
Noboru Kawamoto $^{1}$, Yukihiko Namikawa ${ }^{2, \star}$, Akihiro Tsuchiya ${ }^{3}$, \\ and Yasuhiko Yamada ${ }^{4, \star \star}$ \\ ${ }^{1}$ Department of Physics, Kyoto University, Kyoto 606, Japan \\ ${ }^{2}$ Max-Planck-Institut für Mathematik, D-5300 Bonn, Federal Republic of Germany \\ ${ }^{3}$ Department of Mathematics. Nagoya University, Nagoya 464, Japan \\ ${ }^{4}$ Department of Physics, Nagoya University, Nagoya 464, Japan
}

\begin{abstract}
Conformal field theory on a family of Riemann surfaces is formulated. We derive equations of motion of vacua which are parametrized by moduli of Riemann surfaces and show that these vacua are characterized uniquely by these equations. Our theory has a deep connection with Sato's theory of KP equations.
\end{abstract}

\section{Introduction}

Recently it has been recognized that the conformal field theory (CFT) on Riemann surfaces of arbitrary genus plays an essential role to understand the profound mechanism of the string theory [F.S.; Fr.]. Among others very important insights have been brought by a formulation of the bosonization rule [D.J.K.M.; AG.B.M.N.V.; E.O.; V.V.] and an observation that the Virasoro (energy-momentum tensor) operator deforms the moduli of Riemann surfaces [E.O.; B.M.S.].

One approach to the CFT on Riemann surfaces is based on the path-integral method initiated by Polyakov [P.]. This approach can be regarded as a geometric one which is recently developed into the algebro-geometric level [B.K.].

Another approach to the CFT is an algebraic one based on the representation theory of the Virasoro algebra, and was initiated by Belavin, Polyakov and Zamolodchikov [B.P.Z.]. This approach has an essential connection with solvable models of statistical mechanics and Kac-Moody Lie algebras.

One of the aims of this paper is to unify these two approaches by constructing a CFT on a family of Riemann surfaces in an operator formalism. Another aim is to establish a solid mathematical basis for a class of CFT on Riemann surfaces.

The main ingredient of our theory is M. Sato's theory of KP equations [Sa.; S.S.]. Originally his theory was developed to solve a problem of soliton equations, but here we show that his theory actually covers the CFT on Riemann surfaces.

* Permanent adress: Department of Mathematics, Nagoya University, Nagoya 464, Japan $\star \star$ Adress after April 1, 1988: National Laboratory for High Energy Physics (KEK), Tsukuba, Ibaraki 305, Japan 
Here two notions, the universal Grasmann manifold (UGM) and the $\tau$-function, play the most essential role.

Theory of KP equations was reconstructed by Date, Jimbo, Kashiwara and Miwa in an operator formalism [D.J.K.M.]. Our theory is deeply interrelated with their formulation and is in a sense an extended version to a completed Fock space; in order to treat theta functions we need completion. It should be compared with the treatment of Segal and Wilson [S.W.] which treats the same content in the Hilbert space formalism.

The relation between the theory of KP equations and Riemann surfaces was formulated by Krichever as the theory of the Baker-Akhiezer function [Kr.; Mum. 2], which plays an important role in this paper. This relation to Riemann surfaces was studied further by Mulase and Shiota in connection with the Schottky problem [Mul.; Sh.].

Fundamental operators in our theory are the free fermions $\psi(z), \bar{\psi}(z)$, the current $J(z)$ and the energy-momentum tensor $T(z)$, acting on the Fock space $\mathscr{F}$. They are provided, a priori, without reference to Riemann surfaces. Information of Riemann surfaces are carried by vacuum states $[X] \in \mathbb{P}(\mathscr{F})$ (the projective Fock space). In this respect our theory is based on the interaction picture in physicist's terminology. The space $\hat{\mathscr{C}}$ [respectively $\hat{X}$ which we call the Weierstrass system (Sect. 2)] of all geometric data sets is a dressed moduli space of Riemann surfaces (respectively Riemann surfaces and line bundles). The physical vacuum $[X] \in \mathbb{P}(\mathscr{F})$ moves with a parametrization of data $X \in \hat{\mathscr{C}}$ (or $\hat{\mathscr{X}}$ ). An important fact is that these spaces are infinitesimally homogeneous spaces on which infinite dimensional Lie algebras $\left(\mathfrak{G}=\mathbb{C}\left(\left(z^{-1}\right)\right) \frac{d}{d z}\right.$ and $\widetilde{\mathfrak{G}}=\mathfrak{G} \oplus \mathbb{C}\left(\left(z^{-1}\right)\right)$ act respectively.

Our main viewpoint is to interpret the map $\hat{\mathscr{C}}$ (respectively $\hat{\mathscr{X}}) \rightarrow \mathbb{P}(\mathscr{F}), X \mapsto[X]$ as a period map of the moduli space $\widehat{\mathscr{C}}$ (respectively $\widehat{X}$ ) and is to investigate the deformation of moduli generated by operators $t \in \mathfrak{E}$ (respectively $\tilde{\mathfrak{F}}$ ). An important fact is that the view as a period map matches very well with the method of field theory. For example the fundamental operators $T(z)$ and $J(z)$ are just the deformation generators mentioned above. From a physical point of view this deformation equation can be considered as an equation of motion of the vacua. On the other hand it can be regarded as the Gauss-Manin connection from an algebrogeometric point of view.

We have tried to make this article as self-contained as possible, since no detailed reference on Sato's theory is available in western language. This paper is organized as follows:

Sections 1 and 2 are devoted to the geometrical setup for a description of the period map. In Sect. 1 we give a description of UGM (universal Grassmann manifold) and its Plücker embedding into projective Fock space $\mathbb{P}(\mathscr{F})$, essentially following M. Sato. In Sect. 2 we construct the dressed moduli space of curves $\mathscr{\mathscr { C }}$ (following [B.M.S.]) and its generalization $\hat{\mathscr{X}}$ including the Picard variety, which are infinite dimensional complex manifolds.

Using the theory of the abelian functions, we can define a period map from $\hat{\mathscr{C}}$ (respectively $\hat{\mathscr{X}}$ ) to UGM and derive Torelli-type theorems (2.28), (2.29) as a main result of Sect. 2. The action of the modular group on $\hat{\mathscr{C}}$ (respectively $\hat{\mathscr{X}}$ ) is also important. 
Section 3 is devoted to a preparation of the algebraic setup. In terms of the fermion operator $\psi(z), \bar{\psi}(z)$ acting on the Fock space $\mathscr{F}$, we construct a Fock space representation of a central extension of $(\mathfrak{g}$ (or $\widetilde{\mathfrak{G}}$ ), where the Virasoro algebra and the current algebra appear.

In Sect. 4 we provide the bosonization $B: \mathscr{F} \rightarrow \mathscr{H}$ (bosonized Fock space) and show that the image of UGM in $\mathscr{H}$ can be characterized by a conjugate pair of wave functions and the Hirota equations for $\tau$-functions. We essentially follow [D.J.K.M.] but reformulate the theory in an appropriate form for field theory.

The contents of Sects. 1-4 can be summarized in the following diagram:

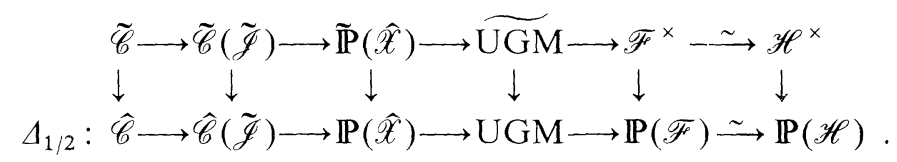

The space $\hat{\mathscr{C}}(\tilde{\mathscr{J}})$ is, stated briefly, the deformation of $\hat{\mathscr{C}}$ in the direction to the Jacobian varieties with a gauge fixing (see Sect. $2, F)$ ). The symbols with " , $\left(\tilde{\mathscr{C}}\right.$ etc.) stand for the induced $\mathbb{C}^{*}$-bundles,

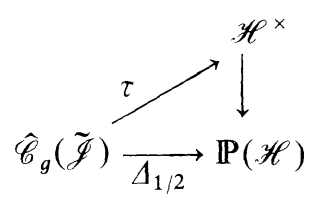

In Sect. 5 we construct a well-behaved lifting which we call the $\tau$-function of the period map $\Delta_{1 / 2}$ with a method based on Krichever's theory concerning the BakerAkhiezer function (i.e. a wave function associated to the curve). This $\tau$-function can be explicitly written down in terms of the classical Riemann theta function and the Jacobian embedding of Riemann surfaces:

$$
\tau\left(\mathbb{T}, X_{c}\right)=e^{\frac{1}{2} q(\mathbb{T})} \Theta(I(\mathbb{T})+c \mid \Omega) .
$$

This explicit form of the $\tau$-function has been already given by several authors [I.M.O.; A-G.G.R.; V.].

In Sect. 6 the explicit forms of actions of fundamental operators on the $\tau$-function and $N$-point functions are obtained by using the concrete expression of the $\tau$-function. It is interesting to note that we can obtain the addition formulae of $\Theta$-functions associated with the Jacobian of a curve systematically, which are closely related with the Schottky problem in algebraic geometry [Fay; Mum. 3; v.G.; Mul.; Sh.].

In Sect. 7 the fundamental differential equations satisfied by the $\tau$-function will be derived. This provides the Gauss-Manin connection of this period map. The main result of this paper can be stated as follows: The $\tau$-function is characterized by the differential linear equations with infinite degrees of freedom:

$$
\begin{array}{cc}
{\left[\theta(\ell)+a\left(\ell, X_{c}\right)\right] \tau\left(\mathbb{T}, X_{c}\right)=\Phi_{B}(\ell) \tau\left(\mathbb{T}, X_{c}\right),} & \forall \ell \in \mathbb{5} \\
\sum_{i=1}^{g} \frac{1}{2 \pi \sqrt{-1}} \int_{\beta_{i}} d \varphi \frac{\partial}{\partial c^{i}} \tau\left(\mathbb{T}, X_{c}\right)=\Phi_{B}(\varphi) \tau\left(\mathbb{T}, X_{c}\right), & \forall \varphi(z) \in \tilde{A}(X),
\end{array}
$$


and together with the automorphy:

$$
\tau\left(\mathbb{T}, X_{c+\Omega a+b}\right)=\exp \left(-2 \pi \sqrt{-1}\left(\frac{1^{t} a}{2} \Omega a+{ }^{t} a(I(\mathbb{T})+c)\right)\right) \tau\left(\mathbb{T}, X_{c}\right) .
$$

Here $X_{c}$ lies in the moduli space $\hat{\mathscr{C}}(\tilde{\mathscr{J}})$ of Riemann surfaces (Sect. 2, F)) and $\mathrm{T}=\left(t_{1}, t_{2}, \ldots\right)$ is the parameter of the bosonic Fock space $\mathscr{H}$ (Sect. 4, A)). For $\ell \in \mathfrak{G}, \theta(\ell)$ stands for its action on $X_{c}$ as a vector field on $\hat{\mathscr{C}}(\tilde{\mathscr{J}})$ (Sect. 2, D)) and $\Phi_{B}(\ell)$ acts on $\mathbb{T}$ as a quantized operator on $\mathscr{H}(3.20),(4.4)$. For the notations in the second type of equations (concerning gauge transformations) see the Appendix. It is a remarkable fact that the $\tau$-function characterized by the above linear equations satisfies Hirota's nonlinear equations.

In the Appendix we collect the notations and the formulae in the theory of abelian functions on Riemann surfaces which we have used for explicit descriptions of our results in this article.

One of the main characteristics of our theory is that we have constructed the whole theory to keep the complex analyticity throughout the formulation. Another characteristic is that any infinite dimensional manifold like $\hat{\mathscr{C}}$ and $\hat{\mathscr{X}}$ is treated as a projective limit of a finite dimensional manifold. The latter treatment is compatible with the completion of the Fock space.

Moreover the structure of our theory has an intimate similarity with a recent theory of arithmetic surfaces due to Arakelov and Faltings [Ar.; Fal.].

Some of the results in Sects. 5 and 6 have already been given by Ishibahsi, Matsuo, and Ooguri [I.M.O.] and Alvarez-Gaumé, Gomez, and Reina [A-G.G.R.]. These sections are, however, reformulated with the help of the preparations of Sects. 1-4 within the framework of the theory of infinite dimensional complex analytic manifolds. Our main contribution in this paper is to clear up the geometric setting of the whole theory and to provide a system of differential equations which characterizes the $\tau$-function uniquely.

After this work has been completed a very interesting paper by Krichever and Novikov [K.N.] came to our attention, which seems to have a close relation with our paper.

\section{UGM and Its Plücker Embedding}

\section{A) Universal Grassmann Manifold (UGM)}

(1.0) The theory of UGM was created and developed by Sato and plays an essential role in his theory of KP equations [Sato; S.S.]. Thanks to the additional structure of filtration one can obtain an almost complete analogy to the theory of usual Grassmann manifolds for finite dimensional spaces [S.N.]. We recall here in Sect. 1 the most elementary part of the theory (or the part which is the same as usual finite dimensional cases). The deeper part where we need an essential modification including the theory of $\tau$-functions will be treated in Sects. 3 and 4 .

(1.1) Let $\mathscr{V}$ be a linear space (over $\mathbb{C}$ ) equipped with a filtration $\left\{F^{m} \mathscr{V}\right\}_{m \in \mathbb{Z}}$ satisfying the following conditions: 
1. $\left\{F^{m} \mathscr{V}\right\}_{m \in \mathbb{Z}}$ is decreasing, ..つ $F^{m} \mathscr{V} \supset F^{m+1} \mathscr{V} \supset \ldots$;

2. $\bigcup_{m \in \mathbb{Z}} F^{m} \mathscr{V}=\mathscr{V}$, and $\bigcap_{m \in \mathbb{Z}} F^{m} \mathscr{V}=\{0\}$;

3. $\operatorname{dim}_{\mathbb{C}} F^{m} \mathscr{V} / F^{m+1} \mathscr{V}=1$;

4. the topology induced from the filtration (i.e. $\left\{F^{m} \mathscr{V}\right\}_{m \in \mathbb{Z}}$ form a system of neighbourhoods of 0 ) is complete.

Such $\mathscr{V}$ is actually unique up to isomorphisms.

(1.2) For concrete calculation we choose a (topological) basis of $\mathscr{V}$ as follows. For later use we employ $\mathbb{Z} h=\mathbb{Z}+(1 / 2)=\{n+(1 / 2) ; n \in \mathbb{Z}\}$ as an index set and choose $e^{\mu}$ in $F^{\mu-(1 / 2)} \mathscr{V}-F^{\mu+(1 / 2)} \mathscr{V}$ for each $\mu \in \mathbb{Z} h$. Then every element $v$ in $\mathscr{V}$ can be expressed as

and

$$
v=\sum_{\substack{-\infty \ll \mu<\infty \\ \mu \in \mathbb{Z} h}} v_{\mu} e^{\mu}, \quad v_{\mu} \in \mathbb{C}
$$

$$
F^{m} \mathscr{V}=\left\{v=\sum_{m<\mu} v_{\mu} e^{\mu}\right\} .
$$

(1.3) Example. $\mathscr{V}=\hat{K} \sqrt{d \zeta}(\hat{K}=\mathbb{C}((\zeta))$ is the field of formal Laurent series $)$. The filtration comes from the non-archimedian valuation $v: \mathscr{V} \rightarrow \overline{\mathbb{Z}}=\mathbb{Z} \cup\{\infty\}$ with $v\left(\zeta^{n} \sqrt{d \zeta}\right)=n$. We use a basis $\left\{e^{m+(1 / 2)}=\zeta^{m} \sqrt{d \zeta}\right\}_{m \in \mathbb{Z}}$. For later use we introduce another indeterminate $z$ with the relation $z \zeta=1$. Namely $z$ is a coordinate whose value tends to $\infty$ at the reference point. When we use this coordinate in $\mathscr{V}=\mathbb{C}\left(\left(z^{-1}\right)\right) \sqrt{d z}$ the valuation is given by $v\left(z^{m} \sqrt{d z}\right)=v\left(\zeta^{-m-1} \sqrt{d \zeta}\right)=-m-1$.

(1.4) Let $\overline{\mathscr{V}}=\operatorname{Hom}_{\mathbb{C}}(\mathscr{V}, \mathbb{C})$ be the topological dual space (namely the space of continuous $\mathbb{C}$-linear functions on $\mathscr{V}$ with discrete topology on $\mathbb{C}$ ). Then $\overline{\mathscr{V}}$ has a canonical dual structure of a filtered space as

$$
F_{m} \overline{\mathscr{V}}=\operatorname{Ker}\left(\operatorname{Hom}(\mathscr{V}, \mathbb{C}) \rightarrow \operatorname{Hom}\left(F^{m} \mathscr{V}, \mathbb{C}\right)\right) .
$$

If we set $F^{m} \overline{\mathscr{V}}=F_{-m} \overline{\mathscr{V}}$, and $F_{m} \mathscr{V}=F^{-m} \mathscr{V}$, then $\left(\overline{\mathscr{V}},\left\{F^{m} \overline{\mathscr{V}}\right\}\right)$ satisfies the same properties as in (1.1) and $\mathscr{V}$ and $\widetilde{V}$ are dual to each other. In particular

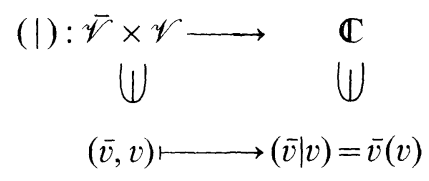

is a complete dual pairing of topological vector spaces.

For a basis $\left\{e^{\mu}\right\}_{\mu \in \mathbb{Z} h}$ of $\mathscr{V}$ the dual basis $\left\{\bar{e}^{\mu}\right\}_{\mu \in \mathbb{Z} h}$ of $\overline{\mathscr{V}}$ can be defined by the relations:

$$
\left(\bar{e}^{\mu} \mid e^{v}\right)=\delta^{\mu+v, 0} .
$$

Then $\bar{e}^{m+(1 / 2)} \in F^{m} \overline{\mathscr{V}}-F^{m+1} \overline{\mathscr{V}}$. Every element $\bar{v} \in F^{m} \overline{\mathscr{V}} \subset \overline{\mathscr{V}}$ can be expressed as

$$
\bar{v}=\sum_{m<\mu} \bar{v}_{\mu} \bar{e}^{\mu}, \quad \bar{v}_{\mu} \in \mathbb{C}
$$


(1.5) For later use we introduce another notation:

$$
e_{\mu}=e^{-\mu}, \quad \bar{e}_{\mu}=\bar{e}^{-\mu}, \quad \mu \in \mathbb{Z} h .
$$

Then the pairing on $\overline{\mathscr{V}} \times \mathscr{V}$ becomes the natural one:

$$
\left(\bar{e}_{\mu} \mid e^{v}\right)=\delta_{\mu}^{v}, \quad\left(\bar{e}^{\mu} \mid e_{v}\right)=\delta_{v}^{\mu} .
$$

(1.6) Definition. A subset $M$ of $\mathbb{Z} h$ is called a Maya-diagram if both $M \cap \mathbb{Z} h>0$ and $M^{c} \cap \mathbb{Z} h_{<0}$ are finite sets, where $\mathbb{Z} h_{>0}=\{\mu \in \mathbb{Z} h ; \mu>0\}$ etc. and $M^{c}$ is the complement of $M$ in $\mathbb{Z} h$. The integer $\chi(M)=\#\left(M \cap \mathbb{Z} h_{>0}\right)-\#\left(M^{c} \cap \mathbb{Z} h_{<0}\right)$ is called the charge or the Euler-characteristic of $M$.

We denote by $\mathscr{M}$ (respectively $\mathscr{M}_{p}$ ) the set of all Maya-diagrams (respectively Maya-diagrams of charge $p$ ).

(1.7) Definition. For $M \in \mathscr{M}_{p}$ we express it with an increasing function $\mu: \mathbb{Z} h_{<p}$ $=\{\mu \in \mathbb{Z} h ; \mu<p\} \rightarrow \mathbb{Z} h$ as

$$
M=\{\ldots, \mu(p-(3 / 2)), \mu(p-(1 / 2)\} .
$$

This uniquely defined $\mu$ is called the characteristic function of $M$. Note that $\mu(v)=v$ for $v \ll \infty$. Therefore the set

$$
\{\mu(v)-v ; v \in \mathbb{Z} h, \mu(v)-v>0\}
$$

is finite and defines a Young diagram $Y(M)$. We say that $Y(M)$ is associated with $M$. The number of boxes

$$
d(M)=\sum_{v}(\mu(v)-v)
$$

is called the degree of $M$.

$M$
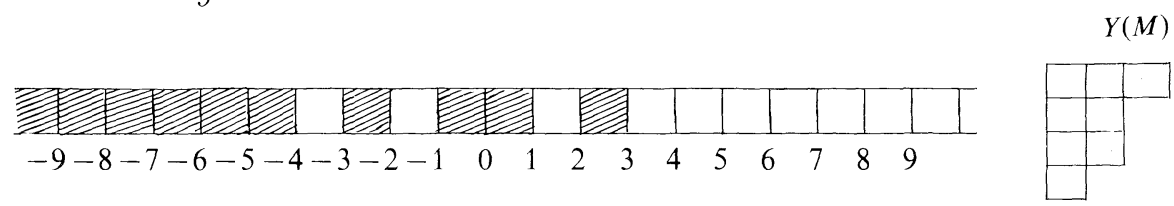

$$
\chi=0, \quad d=8
$$

Denote by $\mathscr{Y}$ (respectively $\mathscr{Y}_{d}$ ) the set of all Young diagrams (respectively Young diagrams of degree $d$ ). Note that \# $\mathscr{Y}_{d}=p(d)$, the number of partitions of $d$.

(1.8) Lemma. We have a canonical bijection

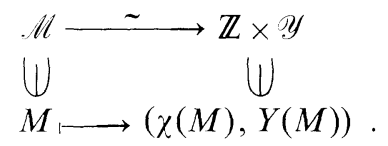

(1.9) Definition. We fix $p \in \mathbb{Z}$. The universal Grassmann manifold (of charge $p$ ) $\mathrm{UGM}^{p}$ is the set of closed subspaces $U$ of $\mathscr{V}$ such that i) the kernel and cokernel of the natural map $f: U \rightarrow \mathscr{V} / F^{0} \mathscr{V}$ is of finite dimension; ii) $\operatorname{dim} \operatorname{Ker}(f)$ $-\operatorname{dim} \operatorname{Coker}(f)=p$. These $U$ 's in $\mathrm{UGM}^{p}$ are called semi-infinite subspaces (of charge $p$ ). Set $\mathrm{UGM}=\coprod_{p \in \mathbb{Z}} \mathrm{UGM}^{p}$. 
Note that, by definition, the topology on $U$, which is induced by $\mathscr{V}$, is discrete. (1.10) By considering the image of $F^{-m} \mathscr{V} \cap U$ in $\mathscr{V} / F^{m} \mathscr{V}, m \in \mathbb{N}$, one can introduce on $\mathrm{UGM}^{p}$ a canonical structure of a scheme as a projective limit of schemes of finite type (Grassmann manifolds for finite dimension), but we will not make it precise here in order to avoid unnecessary complication. Specialists on abstract algebraic geometry could easily fill in logical gaps. For non-specialists it is enough to know that $\mathrm{UGM}^{p}$ admits a "good" structure of an infinite dimensional complex manifold as a "limit" of finite dimensional manifolds.

(1.11) Let $U$ be a closed subspace in $\mathscr{V}$. If we consider the induced filtration $\left\{F^{m} U=F^{m} \mathscr{\gamma} \cap U\right\}$ on $U$, then $\operatorname{dim} F^{m} U / F^{m+1} U \leqq 1$. If we set

$$
M(U)=\left\{m+(1 / 2) \in \mathbb{Z} h ; \operatorname{dim} F^{m} U / F^{m+1} U=1\right\},
$$

then $U \in U G M^{p}$ if and only if $M(U)$ is a Maya-diagram of charge $p$.

We can then define the Young diagram $Y(U)=Y(M(U))$ associated with $U$, and the degree of $U, d(U)=d(M(U))$.

\section{B) Tangent Space of UGM}

(1.12) Proposition [S.N.]. For UEUGM we have a canonical isomorphism

$$
T_{U} \mathrm{UGM} \simeq \operatorname{Hom}_{\mathbb{C}}(U, \mathscr{V} / U)
$$

(here $T_{U} \mathrm{UGM}$ denotes the "holomorphic" tangent space of UGM at $U$ ).

(1.13) Definition. An endomorphism $\varphi$ on $\mathscr{V}$ is a continuous linear map from $\mathscr{V}$ to $\mathscr{V}$ such that there exists an integer $n \in \mathbb{Z}$ with $\varphi\left(F^{m} \mathscr{V}\right) \subset F^{m+n}(\mathscr{V})$ for all $m \in \mathbb{Z}$. Denote by $F^{n} \operatorname{End}(\mathscr{V})$ the set of such endomorphisms and put $\operatorname{End}(\mathscr{V})=\cup F^{n} \operatorname{End}(\mathscr{V})$.

End $\left(\mathscr{V}\right.$ ) is thus a filtered $\mathbb{C}$-algebra (i.e. if $\varphi_{1} \in F^{m}, \varphi_{2} \in F^{n}$, then $\varphi_{1} \circ \varphi_{2} \in F^{m+n}$ ) whose filtration topology is complete.

For each $\varphi \in$ End $(\mathscr{V})$ define $\theta_{U}(\varphi) \in T_{U} \mathrm{UGM}$ by $U \stackrel{i}{\rightarrow} \mathscr{V} \stackrel{\varphi}{\rightarrow} \mathscr{V} \rightarrow \mathscr{V} / U$.

(1.14) Proposition. The linear map $\theta: \operatorname{End}(\mathscr{V}) \rightarrow H^{0}(\mathrm{UGM}, \Theta)=\{$ global holomorphic vector fields on $\mathrm{UGM}\}$ is an anti-homomorphism of Lie algebras, i.e. $\left[\theta\left(\varphi_{1}\right), \theta\left(\varphi_{2}\right)\right]=\theta\left(\left[\varphi_{2}, \varphi_{1}\right]\right)$.

\section{C) Frame Bundle on UGM.}

(1.15) Definition. Let $U \in \mathrm{UGM}^{p}$. A frame $\xi$ of $U$ is a basis of $U$

$$
\xi=\left\{\ldots, \xi^{p-(3 / 2)}, \xi^{p-(1 / 2)}\right\}
$$

such that for $\exists \mu_{0} \in \mathbb{Z} h$ and $\forall \mu \leqq \mu_{0}, \quad \xi^{\mu} \in F^{\mu-(1 / 2)} U-F^{\mu+(1 / 2)} U$ and $\xi^{\mu} \equiv e^{\mu} \bmod F^{\mu+(1 / 2)} U$. (Since the topology of $U$ is discrete, all vectors in $U$ can be expressed as a linear combination of a finite number of vectors in $\xi$.) If we express $\xi$ with $\left\{e^{\mu}\right\}$, then we have

$$
\xi^{r}=e^{v} \xi_{v}^{r} \quad \text { (with Einstein's notation) } v \in \mathbb{Z} h, r \in \mathbb{Z} h_{<p},
$$


or in a form of matrices

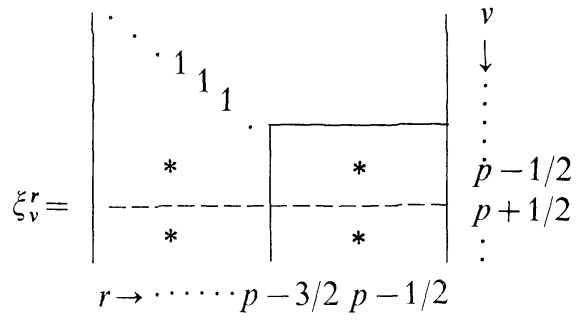

We denote by $\mathrm{FUGM}^{p}$ the set of all frames of elements in $\mathrm{UGM}^{p}$. The natural map $\pi: F U G M \rightarrow U G M$ is holomorphic.

(1.16) Definition. Let $G L\left(\mathbb{Z} h_{<p}\right)$ be a group of semi-finite matrices of the form
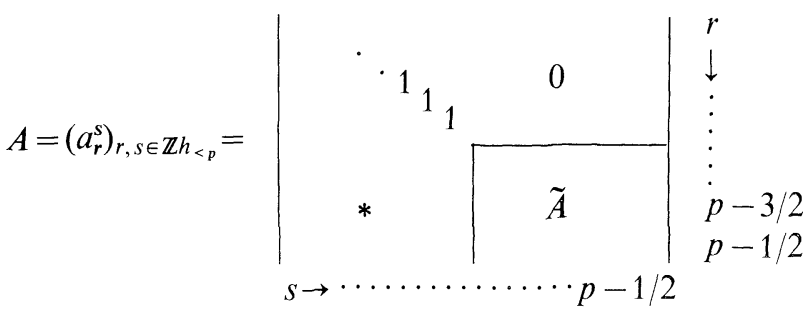

$\operatorname{det} \tilde{A} \neq 0$

For an element $A \in G L\left(\mathbb{Z} h_{<p}\right)$ as above we $\operatorname{define} \operatorname{det}(A)=\operatorname{det}(\tilde{A})$, which is easily seen to be well-defined. We define

$$
S L\left(\mathbb{Z} h_{<p}\right)=\left\{A \in G L\left(\mathbb{Z} h_{<p}\right): \operatorname{det} A=1\right\} .
$$

The group $G L\left(\mathbb{Z} h_{<p}\right)$ operates on $\mathrm{FUGM}^{p}$ from the right as follows :

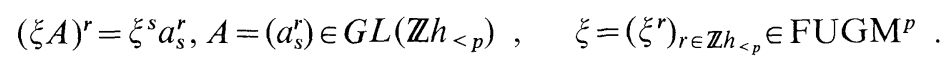

(1.17) Proposition. 1) $\pi: \mathrm{FUGM}^{p} \rightarrow \mathrm{UGM}^{p}$ is a principal $G L\left(\mathbb{Z} h_{<p}\right)$-bundle,

2) ${\widetilde{\mathrm{UGM}^{p}}}^{p} \equiv \mathrm{FUGM}^{p} / S L\left(\mathbb{Z} h_{<p}\right) \rightarrow \mathrm{UGM}^{p}$ is a principal $\mathbb{C}^{*}$-bundle.

(1.18) Definition. Let $G L^{f}(\mathbb{Z} h)$ be the group of invertible linear transformations $g: g\left(e^{\mu}\right)=e^{v} g_{v}^{\mu}$ of $\mathscr{V}$ such that $g_{v}^{\mu}=\delta_{v}^{\mu}$ except for a finite number of $\mu, v$ 's :

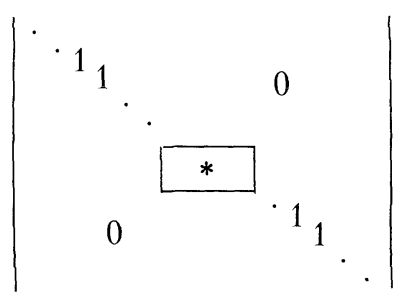

Also $P_{-}=\left(1+\varphi ; \varphi \in F^{1} \operatorname{End}(\mathscr{V})\right)$ is a group since any element $1+\varphi \in P_{-}$is invertible: $(1+\varphi)^{-1}=\sum_{n=0}^{\infty}(-1)^{n} \varphi^{n}$. An element $g \in P_{-}$is represented in the 
following matrix form;

$$
\left(g_{v}^{\mu}\right)=\left|\begin{array}{lllllll}
\cdot & & & & & & \\
& 1 & 1 & & & 0 \\
& & 1 & 1 & & \\
& & & & \ddots & \\
& & & & & \ddots
\end{array}\right|
$$

We denote by $G L_{f}(\mathbb{Z} h)$ the linear transformation group on $\mathscr{V}$ generated by $G L^{f}(\mathbb{Z} h)$ and $P_{-}$. Any element $g$ in $G L_{f}(\mathbb{Z} h)$ can be expressed as

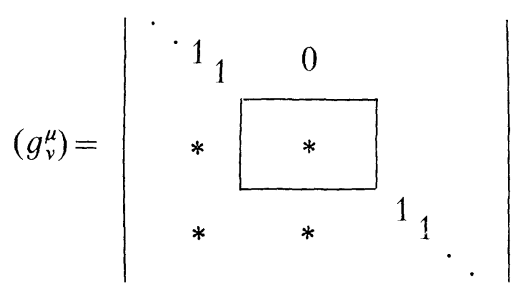

(1.19) Proposition. 1) The group $G L_{f}(\mathbb{Z} h)$ acts on $\mathrm{FUGM}^{p}$ from the left transitively.

2) The action of $G L_{f}(\mathbb{Z} h)$ is commutative with the action of $G L\left(\mathbb{Z} h_{<p}\right)$ :

$(g \xi) A=g(\xi A), \quad$ for $\quad \forall g \in G L_{f}(\mathbb{Z} h), \quad \forall A \in G L\left(\mathbb{Z} h_{<p}\right), \quad \forall \xi \in \mathrm{FUGM}^{p}$.

3) $G L_{f}(\mathbb{Z} h)$ acts on $\widetilde{\mathrm{UGM}^{p}}$ and $\mathrm{UGM}^{p}$.

(1.20) Proposition (Bruhat decomposition) [S.N.]. 1) For $\forall p \in \mathbb{Z}$ and $\forall Y \in \mathscr{Y}$, $\mathrm{UGM}^{p . Y}=\left\{U \in \mathrm{UGM}^{p} ; Y(U)=Y\right\}$ is a locally closed subspace of $\mathrm{UGM}^{p}$, and $P_{-}$ acts on each $\mathrm{UGM}^{p, Y}$ transitively.

2) $\mathrm{UGM}^{p}=\coprod_{Y \in \mathscr{Y}} \mathrm{UGM}^{p, Y}$; disjoint union (Bruhat decomposition).

3) $\mathrm{UGM}^{p, \phi}$ is an open dense subset of $\mathrm{UGM}^{p}$ whose complement is of codimension 1 in $\mathrm{UGM}^{p}$. We call this subspace $\mathrm{UGM}^{p, \phi}$ the big cell (in $\mathrm{UGM}^{p}$ ). We denote $\mathrm{UGM}^{0, \phi}$ simply by $\mathrm{UGM}^{\phi}$.

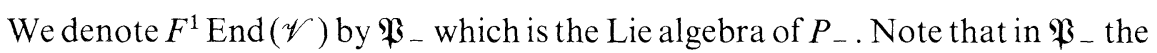
Lie antihomomorphism $\theta$ in (1.14) lifts to

$$
\tilde{\theta}: \mathfrak{P}_{-} \rightarrow H^{0}(\widetilde{\mathrm{UGM}}, \Theta), \quad \varphi \mapsto \tilde{\theta}(\varphi) .
$$

In general it is necessary to "subtract an infinity" when we lift the action of $\varphi \in \operatorname{End}(\mathscr{V})-\mathfrak{B}$ - to that on UGM. This procedure will be treated in Sect. 3.

\section{D) Fock Space (Semi-Infinite Form)}

(1.22) For each Maya-diagram $M=\{\ldots, \mu(p-(3 / 2)), \mu(p-(1 / 2))\}$ we denote by $e^{M}$ or $|M\rangle$ the symbol

$$
e^{\mu(p-(1 / 2))} \wedge e^{\mu(p-(3 / 2))} \wedge \ldots
$$


We consider also finite permutations of $\mu(v)$ 's, under which $e^{M}$ is multiplied by \pm 1 as usual.

(1.23) Definition. The Fock space (of charge $p$ ) is a direct product

$$
\mathscr{F}_{p}=\prod_{M \in \mathscr{M}_{p}} \mathbb{C} e^{M}
$$

and the whole Fock space is

$$
\mathscr{F}=\underset{p}{\oplus} \mathscr{F}_{p}
$$

We define two fundamental operators on $\mathscr{F}$, the charge operator and the mass operator as

$$
\begin{array}{ll}
J_{0}(\alpha)=p \alpha \quad \text { if } \quad \alpha \in \mathscr{F}_{p}, \\
M(\alpha)=d \alpha \quad \text { if } \quad \alpha=e^{M} \quad \text { with } \quad d(M)=d .
\end{array}
$$

They commute each other $\left(\left[J_{0}, M\right]=0\right)$. If we set

$$
\mathscr{F}_{p}(d)=\left\{\alpha \in \mathscr{F} ; J_{0}(\alpha)=p \alpha, M(\alpha)=d \alpha\right\} \quad,
$$

we obtain

Note that

$$
\mathscr{F}=\underset{p}{\oplus} \prod_{d} \mathscr{F}_{p}(d)
$$

$$
\mathscr{F}_{p}(d)=\underset{\chi(M)=p, d(M)=d}{\bigoplus} \mathbb{C} e^{M}
$$

and it is of dimension $p(d)$ [cf. (1.7)].

We define moreover the energy operator as

$$
L_{0}=\frac{1}{2} J_{0}^{2}+M
$$

On $\mathscr{F}_{p}(d), L_{0}$ is a scalar operator; $L_{0}=\left(\frac{1}{2} p^{2}+d\right) i d$.

We introduce a complete Hausdorff linear topology on $\mathscr{F}_{p}$ by the filtration $F^{d}\left(\mathscr{F}_{p}\right)=\prod_{d^{\prime} \geqq d} \mathscr{F}_{p}\left(d^{\prime}\right) ; \ldots \supseteq F^{d}\left(\mathscr{F}_{p}\right) \supseteq F^{d+1}\left(\mathscr{F}_{p}\right) \supseteq \ldots$

(1.24) For a Maya-diagram $M$ as above we define the (dual) symbol $\bar{e}_{M}$ by

$$
\bar{e}_{M}=\ldots \wedge \bar{e}_{\mu(p-(3 / 2))} \wedge \bar{e}_{\mu(p-(1 / 2))},
$$

which is also denoted by $\langle M|$.

Then we define the dual Fock space as a direct sum with discrete topology:

$$
\overline{\mathscr{F}}=\underset{M \in \mathscr{M}_{p}}{\oplus} \mathbb{C} \bar{e}_{M}
$$

We can call it "dual" since we have a continuous bi-linear map

$$
\begin{gathered}
\langle 1\rangle: \overline{\mathscr{F}} \times \mathscr{F} \longrightarrow \mathbb{C} \\
\bigcup \bigcup U \\
\left(\bar{e}_{M}, e^{N}\right) \mapsto\langle M \mid N\rangle=\delta_{M}^{N},
\end{gathered}
$$


by which $\overline{\mathscr{F}}$ and $\mathscr{F}$ are topologically dual to each other (with discrete topology on $\mathbb{C}$ ).

Any element $\alpha$ in $\mathscr{F}$ can be written as

(1.25) We set

$$
\alpha=\sum_{M}\left\langle\bar{e}_{M} \mid \alpha\right\rangle e^{M}=\sum_{M} \alpha_{M} e^{M}
$$

$$
\begin{aligned}
& |p\rangle=e^{p-(1 / 2)} \wedge e^{p-(3 / 2)} \wedge \ldots, \\
& \langle p|=\ldots \wedge \bar{e}_{p-(3 / 2)} \wedge \bar{e}_{p-(1 / 2)}=\ldots \wedge \bar{e}^{-p+(3 / 2)} \wedge \bar{e}^{-p+(1 / 2)} .
\end{aligned}
$$

Then $\mathscr{F}_{p}(0)=\mathbb{C}|p\rangle$.

(1.26) Proposition. The group $G L_{f}(\mathbb{Z} h)$ acts (topologically) on $\mathscr{F}$ by

$$
\begin{aligned}
& \begin{array}{ccc}
G=G[g]: & \mathscr{F} & \longrightarrow \\
\bigcup & & \mathscr{F} \\
& & \bigcup
\end{array} \\
& e^{\mu(p-(1 / 2))} \wedge e^{\mu(p-(3 / 2))} \wedge \ldots \\
& \mapsto g\left(e^{\mu(p-(1 / 2))}\right) \wedge g\left(e^{\mu(p-(3 / 2))}\right) \wedge \ldots
\end{aligned}
$$

for $g \in G L_{f}(\mathbb{Z} h)$, where $g\left(e^{*}\right) \wedge \ldots$ is understood according to the rules of usual exterior algebra.

Proof. For each $M \in M_{p}$,

$$
G\left(e^{M}\right)=\sum_{N \in M_{p}} G_{N}^{M} e^{N}
$$

where

with

$$
G_{N}^{M}=\left\langle\bar{e}_{N} \mid G\left(e^{M}\right)\right\rangle=\operatorname{det}\left(\left(a_{v(\alpha)}^{\mu(\beta)}\right)_{\alpha<p, \beta<p}\right)
$$

$$
M=\{\ldots, \mu(p-(3 / 2)), \mu(p-(1 / 2))\}, \quad N=\{\ldots, v(p-(3 / 2)), v(p-(1 / 2))\} .
$$

Since $g$ belongs $G L_{f}(\mathbb{Z} h)$, the determinant of the infinite matrix is well defined.

(1.27) Corollary. The group $G L_{f}(\mathbb{Z} h)$ acts also on $\overline{\mathscr{F}}$ as $\bar{G}=\bar{G}[g] \in \operatorname{End}(\overline{\mathscr{F}})$ with the condition that

$$
\left\langle\bar{G}(\bar{\alpha}) \mid G\left(\alpha^{\prime}\right)\right\rangle=\left\langle\alpha \mid \alpha^{\prime}\right\rangle
$$

for $\forall \bar{\alpha} \in \overline{\mathscr{F}}, \alpha^{\prime} \in \mathscr{F}$.

(1.28) Remark. One can write $\bar{G}(\bar{\alpha})$ explicitly as above by using the matrix representation of $g^{-1}$.

\section{E) Plücker Embedding}

(1.29) We consider $\xi=\left\{\ldots, \xi^{p-(3 / 2)}, \xi^{p-(1 / 2)}\right\} \in \mathrm{FUGM}^{p}$. Then we see that

$$
\wedge \xi=\xi^{p-(1 / 2)} \wedge \xi^{p-(3 / 2)} \wedge \ldots \in \mathscr{F}_{p}^{\times}=\mathscr{F}_{p}-\{0\} .
$$


Hence we obtain a holomorphic map

$$
\mathrm{FUGM}^{p} \rightarrow \mathscr{F}_{p}
$$

(1.30) Proposition. For $\xi \in \mathrm{FUGM}^{p}$ and $A \in G L\left(\mathbb{Z} h_{<p}\right)$ we have

$$
\wedge(\xi A)=\operatorname{det} A \cdot(\wedge \xi) \text {. }
$$

(1.31) Corollary. We have a commutative diagram of holomorphic maps

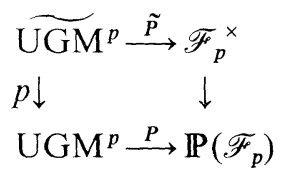

with which the action of $G L_{f}(\mathbb{Z} h)$ is compatible, where $\mathbb{P}\left(\mathscr{F}_{p}\right)$ is the projective space $\mathscr{F}_{p}{ }^{\times} / \mathbb{C}^{*}$ associated with $\mathscr{F}_{p}$.

(1.32) Definition. The map $P: \mathrm{UGM}^{p} \rightarrow \mathbb{P}\left(\mathscr{F}_{p}\right)$ is called the Plücker embedding of $\mathrm{UGM}^{p}$ in the Fock space.

(1.33) Theorem. [S.S.; S.N.]. 1) $P$ is injective and $d p_{U}: T_{U} \mathrm{UGM}^{p} \rightarrow \mathrm{T}_{P(U)} \mathbb{P}\left(\mathscr{F}_{p}\right)$ is also injective for $\forall U \in \mathrm{UGM}^{p}$.

2) The image of $P$ is a closed submanifold in $\mathbb{P}\left(\mathscr{F}_{p}\right)$ defined by the Plücker relations.

\section{Moduli of Riemann Surfaces and Line Bundles}

(2.0) In this section we prepare a geometric framework of our theory. The key idea, due to [B.M.S.], is to introduce an apparently redundant parameter, a (formal) local coordinate, which leads us to work on infinite dimensional manifolds such as the moduli space of locally framed Riemann surfaces. Generalizing [B.M.S.], we construct moduli spaces to include Picard varieties, in order to realize current operators in our geometric settings. The theory then becomes more natural by his generalization. This gigantic moduli space, which we call the Weierstrass system, can be embedded into UGM, and is later identified with the space of physical vacua.

\section{A) Field of Formal Laurent Series}

(2.1) Here we fix a number of notations concerning universal coordinates.

$$
\begin{aligned}
& \hat{K}=\mathbb{C}((\zeta))=\left\{\sum_{n \gg-\infty} a_{n} \zeta^{n} ; a_{n} \in \mathbb{C}\right\}: \text { the field of formal Laurent series, } \\
& \hat{\mathcal{O}}=\mathbb{C}[[\zeta]]=\left\{\sum_{n=0}^{\infty} a_{n} \zeta^{n}\right\}: \text { the ring of formal power series, } \\
& \hat{\mathrm{m}}=\zeta \hat{\mathcal{O}}=\left\{\sum_{n=1}^{\infty} a_{n} \zeta^{n}\right\}: \text { the unique maximal ideal of } \hat{\mathcal{O}} .
\end{aligned}
$$


(2.2) In $\hat{K}$ we define a decreasing filtration satisfying the properties in (1.1) by

$$
F^{n} \hat{K}=\left\{\sum_{k=n}^{\infty} a_{k} \zeta^{k}\right\}
$$

This filtration is the one induced from the unique (non-archimedian) valuation $v: \hat{K} \rightarrow \overline{\mathbb{Z}}=\mathbb{Z} \cup\{\infty\}$ with $v\left(\zeta^{n}\right)=n$ as

$$
F^{n} \hat{K}=v^{-1}([n, \infty])
$$

We have moreover

$$
F^{0} \hat{K}=\hat{\mathcal{O}}, \quad F^{1} \hat{K}=\hat{\mathrm{m}}, \quad F^{n} \hat{K}=\hat{\mathrm{m}}^{n}(n>0) .
$$

(2.3) Let $G_{0}=\operatorname{Aut}_{\mathbb{C}}(\hat{\mathcal{O}})$ be the group of automorphisms of $\hat{\mathcal{O}}$ as $\mathbb{C}$-algebra, which we call coordinate transformations. We have a natural bijection:

$$
\begin{aligned}
& G_{0} \stackrel{\sim}{\longrightarrow} \hat{\mathrm{m}}-\hat{\mathrm{m}}^{2} \\
& W \quad \bigcup \\
& \varphi \mapsto \varphi(\zeta)=b_{1} \zeta+b_{2} \zeta^{2}+\ldots, \quad b_{1} \neq 0 .
\end{aligned}
$$

Let $G_{n}(n \geqq 1)$ be the subgroup of $G_{0}$ consisting of $\varphi$ 's with

$$
\varphi(\zeta)=\zeta+b_{n+1} \zeta^{n+1}+\ldots
$$

(2.4) Consider $\hat{\mathcal{O}}^{*}=\hat{\mathcal{O}}-\hat{\mathrm{m}}=\left\{\sigma=a_{0}+a_{1} \zeta+\ldots ; a_{0} \neq 0\right\}$ which acts on $\hat{\mathcal{O}}$ by multiplication. We call the elements of $\hat{\mathcal{O}}^{*}$ gauge transformations. Denote by $\hat{\mathcal{O}}_{n}^{*}$ the subgroup of elements $\sigma$ whith $\sigma=1+a_{n} \zeta^{n}+\ldots$.

We define a semidirect product group $\widetilde{G}_{0}=G_{0} \times \hat{\mathcal{O}}^{*}$ by commuting relations $\varphi \circ \sigma=\varphi(\sigma) \circ \varphi$ for $\varphi \in G_{0}, \sigma \in \hat{\mathcal{O}}$.

Then $\widetilde{G}_{0}$ acts on $\hat{\mathcal{O}}$ as $\mathbb{C}$-linear endomorphisms by $(\varphi, \sigma)(f)=\varphi(\sigma \cdot f)$. Note that $(\varphi, \sigma)$ is uniquely determined by its values at 1 and $\zeta$. We set moreover $\widetilde{G}_{n}=G_{n} \times \hat{\mathcal{O}}_{n}^{*}$. Then $\widetilde{G}_{1}$ is a normal subgroup of $\tilde{G}_{0}$. (2.5) Let $\left(\mathfrak{5}=\operatorname{Der}(\hat{K})=\hat{K} \frac{d}{d \zeta}\right.$ be the Lie-algebra of derivations on $\hat{K}$. Define its
filtration by

$$
\begin{aligned}
& F^{n} \mathfrak{G}= \mathfrak{G}_{n}=\left\{\ell \in \mathfrak{G}: F^{m} \hat{K} \rightarrow F^{m+n} \hat{K}\right\}=\hat{\mathfrak{O}} \zeta^{n+1} \frac{d}{d \zeta} \\
& \ldots \supseteq \mathfrak{G}_{n} \supseteq \mathfrak{G}_{n+1} \supseteq \ldots
\end{aligned}
$$

\section{(2.6) Proposition.}

1) $\left[\mathfrak{G}_{n}, \mathfrak{G}_{m}\right] \subseteq \mathfrak{G}_{n+m}$,

2) $\operatorname{dim}\left(\mathfrak{5}_{n} / \mathfrak{6}_{n+1}=1\right.$,

3) $\boldsymbol{G}_{n}(n \geqq-1)$ is a subalgebra of $(\mathfrak{5}$.

Note that $\mathfrak{5}_{n}=\operatorname{Lie} G_{n}$ for $n \geqq 0$ but $\mathfrak{5}_{-1}$ does not correspond to any holomorphic Lie group. We denote $\mathfrak{G}_{0}=\mathfrak{B}, \mathfrak{b}_{1}=\mathfrak{B}_{\text {- }}$ in the following. 
(2.7) Let $\widetilde{\mathfrak{G}}=\mathscr{D}^{1}(\hat{K})=\hat{K} \frac{d}{d \zeta}+\hat{K}$ be the Lie algebra of differential operators of order $\leqq 1$ on $\hat{K}$. Then it satisfies the following commutation relation for $\ell_{i} \frac{d}{d \zeta}+s_{i} \in \tilde{\mathfrak{G}}(i=1,2)$ :

$$
\left[\ell_{1} \frac{d}{d \zeta}+s_{1}, \ell_{2} \frac{d}{d \zeta}+s_{2}\right]=\left(\ell_{1} \frac{d \ell_{2}}{d \zeta}-\ell_{2} \frac{d \ell_{1}}{d \zeta}\right) \frac{d}{d \zeta}+\left(\ell_{1} \frac{d s_{2}}{d \zeta}-\ell_{2} \frac{d s_{1}}{d \zeta}\right) .
$$

Define its filtration by

$$
\begin{aligned}
F^{n} \widetilde{\mathfrak{G}}= & \widetilde{\mathfrak{W}}_{n}=\left\{D \in \widetilde{\mathfrak{G}}: F^{m} \hat{K} \rightarrow F^{m+n} \hat{K}\right\}=\hat{\mathcal{O}} \zeta^{n+1} \frac{d}{d \zeta} \oplus \hat{\mathcal{O}} \zeta^{n} \\
& \ldots \supseteq \widetilde{\mathfrak{G}}_{n} \supseteq \widetilde{\mathfrak{W}}_{n+1} \supseteq \ldots .
\end{aligned}
$$

(2.8) Proposition. 1) $\left[\widetilde{\mathfrak{G}}_{n}, \widetilde{\mathfrak{G}}_{m}\right] \subseteq \widetilde{\mathfrak{G}}_{n+m}$,

2) $\widetilde{\mathfrak{F}}_{n}(n \geqq 0)$ is a subalgebra of $\widetilde{\mathfrak{G}}$.

Note that $\widetilde{\mathfrak{F}}_{n}=$ Lie $\widetilde{G}_{n}(n \geqq 0)$. In the following, we denote $\widetilde{\mathfrak{G}}_{0}=\mathfrak{B} \oplus \mathfrak{H}$ and $\widetilde{\mathfrak{b}}_{1}=\widetilde{\mathfrak{G}}_{-}=\mathfrak{B}_{-} \oplus \mathfrak{U}_{-}$, where $\mathfrak{\mathfrak { H }}=\operatorname{Lie} \hat{\mathcal{O}}^{*}=\hat{\mathcal{O}}$ and $\mathfrak{H}_{-}=\operatorname{Lic} \hat{\mathcal{O}}_{1}^{*}=\hat{m}$.

(2.9) Remark. The algebra $\widetilde{\mathfrak{G}}_{0}$ acts on $\hat{\mathcal{O}}$ as

$$
\left(\ell \frac{d}{d \zeta}+s\right)(f)=\ell \frac{d f}{d \zeta}+s f .
$$

(2.10) Proposition. The exponential map exp : $\widetilde{\mathfrak{G}}_{0} \rightarrow \widetilde{G}_{0}$ sending $D \in \widetilde{\mathfrak{G}}_{0}$ to $e^{D} \in \widetilde{G}_{0}$ is well-defined and surjective, and it induces a bijection $\exp : \widetilde{\mathfrak{G}}_{1} \rightarrow \widetilde{G}_{1}$.

(2.11) Remark. Later we use mainly a coordinate $z=\zeta^{-1}$ (i.e. $\left.\hat{K}=\mathbb{C}\left(\left(z^{-1}\right)\right)\right)$ to adapt our notations to the convention in physics. Since $z \frac{d}{d z}=-\zeta \frac{d}{d \zeta}$, the feature of $(5)$ does not change much:

$$
\begin{aligned}
& \mathfrak{W}_{n}=\mathbb{C}\left[\left[z^{-1}\right]\right] z^{-m+1} \frac{d}{d z} \\
& \widetilde{\mathfrak{W}}_{n}=\mathbb{C}\left[\left[z^{-1}\right]\right] z^{-n+1} \frac{d}{d z} \oplus \mathbb{C}\left[\left[z^{-1}\right]\right] z^{-n} .
\end{aligned}
$$

\section{B) Riemannn Surfaces and Their Picard Varieties}

(2.12) We recall some elementary notions on Riemann Surfaces. For details we refer the reader to [Si.; F.K.].

(2.13) Let $R$ be a Riemann surface of genus $g \geqq 0$ and $K=K(R)$ the field of meromorphic functions on $R$. Note that $K$ determines $R$ uniquely up to isomorphisms.

Let $Q$ be a point on $R$. Denote by $\mathcal{O}_{Q}$ the ring of meromorphic functions on $R$ which are holomorphic at $Q . \mathrm{m}_{Q}$ is the unique maximal ideal of $\mathcal{O}_{Q}$ consisting of meromorphic functions vanishing at $Q$. 
There is a valuation $v_{Q}$ at $Q$ in $K$ such that $v_{Q}(f)=$ the order of zero at $\mathcal{E}=$-the order of pole at $Q$, which defines a filtration $\left\{F_{Q}^{n} K\right\}$ in $K$. This $v_{Q}$ determines a point $Q$ with the relation $\mathcal{O}_{Q}=\left\{f \in K ; v_{Q}(f) \geqq 0\right\}$. We further define $\hat{K}_{Q}=\underbrace{\lim }_{n} K / \mathfrak{m}_{Q}^{n+1} \mathcal{O}_{Q}$ (the completion of $K$ with respect to $\mathfrak{m}_{Q}$-adic topology).

(2.14) Definition. We denote by $A(Q)=A(R, Q)$ the space $H^{0}(R, \mathcal{O}(* Q))$ $=\{f \in K$; holomorphic except at $Q\}$.

This is a subring in $K$ such that $A(Q) \cap \mathcal{O}_{Q}=\mathbb{C} 1$. It has a filtration $\left\{F^{n} A(Q)\right)$ induced from $\left\{F_{Q}^{n} K\right\}$ in $K$.

(2.15) More generally let $\mathscr{L}$ be (a sheaf of germs of holomorphic sections of) a line bundle on $R, \mathscr{L}_{Q}$ the vector space of meromorphic sections of $\mathscr{L}$ which are holomorphic at $Q$. Note that $\mathscr{L}_{Q}$ is an $\mathcal{O}_{Q}$-module, free of rank 1 .

We denote by $H^{0}(\mathscr{L}(* Q))$ the space of meromorphic sections of $\mathscr{L}$ on $R$ which are holomorphic except at $Q$. It has a filtration:

$$
F^{m} H^{0}(\mathscr{L}(* Q))=\left\{f \in H^{0}(\mathscr{L}(* Q)) \text { : order of zero at } Q \geqq m\right\}, m \in \mathbb{Z} .
$$

In particular we set:

$\bar{B}(R, Q)=H^{0}(R, \Theta(* Q))=\{$ meromorphic vector fields, holomorphic outside $Q\}$, where $\Theta$ is the sheaf of germs of holomorphic vector fields on $R$.

(2.16) The set $P=\operatorname{Pic}(R)$ of holomorphic line bundles on $R$ has a natural structure of an abelian Lie group. Its connected components are parametrized by the degree $d$ (i.e. the first Chern class) or the Euler-characteristic $\chi$ :

$$
P=\coprod_{d \in \mathbb{Z}} P^{(d)}=\coprod_{\chi \in \mathbb{Z}} P^{\chi}, \quad \chi=d+1-g .
$$

The connected component $P^{(0)}$ of 0 (i.e. the trivial line bundle) is an abelian variety $J$ $=J(R)$ called the Jacobian variety of $R$. If we choose a line bundle $\mathscr{L}_{1} \in P^{(1)}$, we have an isomorphism of groups $J(R) \times \mathbb{Z} \stackrel{\sim}{\longrightarrow} P$ sending $(\mathscr{L}, d)$ to $\mathscr{L} \otimes \mathscr{L}_{1}^{\otimes d}$ (with the convention: $\mathscr{L}_{1}^{\otimes(-1)}=\mathscr{L}_{1}^{\vee}$, where $\mathscr{L}_{1}^{\vee}$ denotes the dual of $\left.\mathscr{L}\right)$.

$P$ has a canonical involution (charge conjugation) $*: P^{\chi} \rightarrow P^{-\chi}$ sending $\mathscr{L}$ to $\Omega \otimes \mathscr{L}^{\vee}$, where $\Omega\left(=\Theta^{\vee}\right)$ is the cotangent bundle.

\section{C) Formal Uniformization}

(2.17) Let $R$ be a Riemann surface. Denote by $\hat{R}$ the set of isomorphisms (as $\mathbb{C}$-algebras) $u: \hat{\mathcal{O}}_{Q} \stackrel{\sim}{\longrightarrow} \hat{\mathcal{O}}=\mathbb{C}[[\zeta]]$ (2.1) with $Q \in R$, where $\hat{\mathcal{O}}_{Q}$ denotes the completion $\lim _{n} \mathcal{O}_{Q} / m_{Q}^{n+1}$ of $\mathcal{O}_{Q}$. A specification of $u$ is equivalent to give a valuationpreserving $\mathbb{C}$-algebra homomorphism $\mathcal{O}_{Q} \rightarrow \hat{\mathcal{O}}$. If moreover the image of $u: \hat{O}_{Q} \rightarrow \hat{\mathcal{O}}$ is contained in the subring of convergent power series $\mathcal{O} \subset \hat{\mathcal{O}}$, then the specification of $u$ is nothing but to choose a holomorphic coordinate at $Q$. Since $K$ is the quotient field of $\mathcal{O}_{Q}, u: \mathcal{O}_{Q} \rightarrow \hat{\mathcal{O}}$ induces an injective $\mathbb{C}$-algebra homomorphism $u: K \rightarrow \hat{K}$ which preserves the valuations and further induces an isomorphism $u: \hat{K}_{Q} \rightarrow \hat{K}$. Such $u$ is called a formal uniformization at $Q$. 
There is a natural surjective map $\tilde{\omega}: \hat{R} \rightarrow R$ sending $u$ to $Q$. This $\hat{R}$ has a canonical structure of an infinite dimensional complex manifold as a projective limit of finite dimensional ones: $R^{(n)}=\left\{u_{n}: \mathcal{O}_{Q} / \mathrm{m}_{Q}^{n+1} \stackrel{\sim}{\longrightarrow} \mathcal{O} / \hat{\mathrm{m}}^{n+1}\right\}$.

On $R$ the group $G_{0}=\operatorname{Aut}_{\mathbb{C}}(\hat{\mathcal{O}})$ acts (from the left) as $u \rightarrow \varphi \circ u$ for $\varphi \in G_{0}, u \in R$. With this action $R$ becomes a (holomorphic) principal fibre space over $R$ with $G_{0}$ as fibres. For $n \geqq 1, R^{(n)} \rightarrow R$ becomes a principal fibre space over $R$ with $G^{(n)}=G_{0} / G_{n}$ as

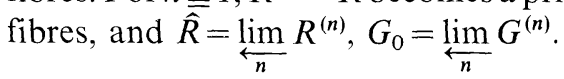

(2.18) Let $(R,(\alpha, \beta))$ be a (homologically) marked Riemann surface with a canonical basis $\left\{\alpha_{1}, \ldots, \alpha_{g}, \beta_{1} \ldots, \beta_{g}\right\}$ of $H_{1}(R, \mathbb{Z})$ (i.e. $\left.\left(\alpha_{i}, \beta_{j}\right)=\delta_{i j},\left(\alpha_{i}, \alpha_{j}\right)=\left(\beta_{i}, \beta_{j}\right)=0\right)$. $(R,(\alpha, \beta))$ and $\left(R^{\prime},\left(\alpha^{\prime}, \beta^{\prime}\right)\right)$ are isomorphic if there exist an isomorphism $f: R \rightarrow R^{\prime}$ such that $f_{*}\left(\alpha_{i}\right)=\alpha_{i}^{\prime}, f_{*}\left(\beta_{i}\right)=\beta_{i}^{\prime}\left(f_{*}: H_{1}(R) \rightarrow H_{1}\left(R^{\prime}\right)\right)$. Denote the set of isomorphism classes of $(R,(\alpha, \beta))$ by $\mathscr{T}_{g}$. It admits a canonical structure of a complex manifold of dimension $0(g=0), 1(g=1), 3 g-3(g \geqq 2)$. We call this $\mathscr{T}_{g}$, the Torelli space.

On $\mathscr{T}_{g}$ the modular group $M(=S p(2 g, \mathbb{Z}))$ acts properly discontinuously (as base changes in $H_{1}(R, \mathbb{Z})$ ) and the quotient space $\mathscr{T}_{g} / M$ is isomorphic to the coarse moduli space $\mathscr{M}_{g}$ of Riemann surfaces of genus $g$ (i.e. the set of isomorphism classes). Over $\mathscr{T}_{g}$ there is a universal family of Riemann surfaces $\pi: \mathscr{C}_{g} \rightarrow \mathscr{T}_{g}$, where $\mathscr{C}_{g}=\bigcup_{([R],(\alpha \beta)) \in \mathscr{T}_{g}} R$ (see Fig. 1).

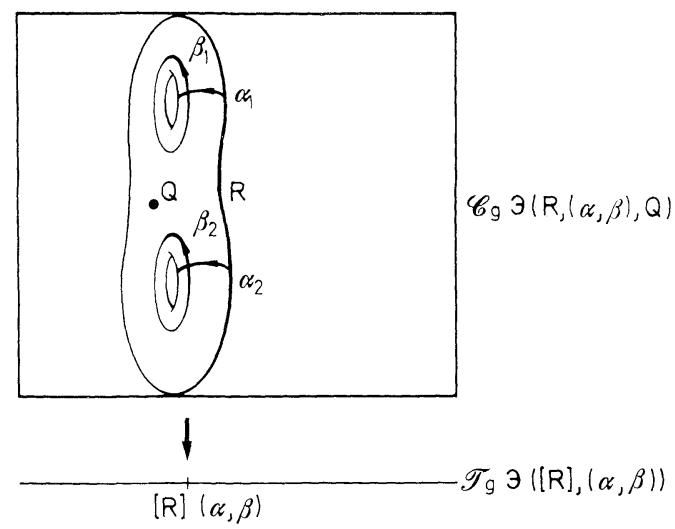

Fig. 1

What we need here essentially is that the tangent space $T_{[R]} \mathscr{T}_{g}$ of $\mathscr{T}_{g}$ at $[R]$ is canonically isomorphic to $H^{1}\left(R, \Theta_{R}\right)$ via the Kodaira-Spencer map [Ko.]. Then for any $Q \in R$ the tangent space of $\mathscr{C}_{g}$ at $Q$ is canonically isomoprhic to

$$
\begin{aligned}
& T_{Q} \mathscr{C}_{g} \stackrel{\sim}{\longrightarrow} H^{1}\left(R, \mathrm{~m}_{Q} \Theta_{R}\right)=H^{1}\left(R, \Theta_{R}(-Q)\right), \quad(g \geqq 2), \\
& T_{Q} \mathscr{C}_{g} / T_{Q}\left(\mathscr{C}_{g} / \mathscr{T}_{g}\right) \stackrel{\sim}{\longrightarrow} H^{1}\left(R, \mathrm{~m}_{Q} \theta_{R}\right)=H^{1}\left(R, \Theta_{R}\right)(g=0,1),
\end{aligned}
$$

where $T_{Q}\left(\mathscr{C}_{g} / \mathscr{T}_{g}\right)$ is the tangent space at $Q$ of the fibre of $\pi: \mathscr{C}_{g} \rightarrow \mathscr{T}_{g}$.

For the universal family of Riemann surfaces $\pi: \mathscr{C}_{g} \rightarrow \mathscr{T}_{g}$, the union of

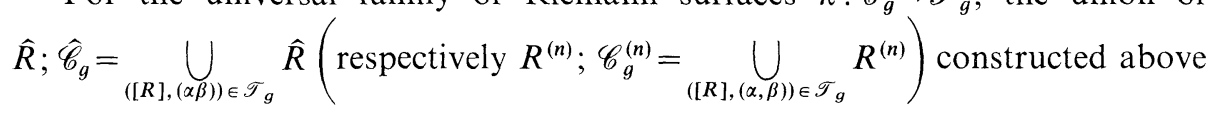


admits a natural structure of a holomorphic family

$$
\tilde{\omega}: \hat{\mathscr{C}}_{g} \rightarrow \mathscr{C}_{g} \quad \text { (respectively } \tilde{\omega}_{n}: \mathscr{C}_{g}^{(n)} \rightarrow \mathscr{C}_{g} \text { ), }
$$

which are again fibre bundles with structure group $G_{0}$ (respectively $G^{(n)}$ ). For convenience we set $\mathscr{C}_{g}^{(0)}=\mathscr{C}_{g}$. Then we have

$$
T_{\left(Q, u_{n}\right)}\left(\mathscr{C}_{g}^{(n)} / \operatorname{Aut}^{0}(R)\right) \stackrel{\sim}{\longrightarrow} H^{1}\left(R, \Theta_{R}((-n-1) Q)\right) \quad(n \geqq 0)
$$

For each data $X=\left(R,(\alpha, \beta), Q, u: \hat{\mathcal{O}}_{Q} \rightarrow \hat{\mathcal{O}}\right) \in \hat{\mathscr{C}}_{g}$, we define a $\mathbb{C}$-subalgebra of $\hat{K}$, $A(X)$, by $u A(R, Q)$ and Lie subalgebras $\bar{B}(X)$ of $\operatorname{Der}(\hat{K})=\hat{K} \frac{d}{d \zeta}$ by $u \bar{B}(R, Q)$ respectively [cf. (2.14), (2.15)].

(2.19) Proposition [B.M.S.1]. 1) We have canonical isomorphisms:

$$
\begin{aligned}
& H^{1}\left(R, \Theta_{R}\right) \stackrel{\sim}{\sim} \operatorname{Der}(\hat{K}) /\left(\hat{\mathcal{O}} \frac{d}{d \zeta}+\bar{B}(X)\right), \\
& H^{1}\left(R, \Theta_{R}((-n-1) Q)\right) \stackrel{\sim}{\operatorname{Der}}(\hat{K}) /\left(\hat{\mathcal{O}} \zeta^{n+1} \frac{d}{d \zeta}+\bar{B}(X)\right)
\end{aligned}
$$

at $X=(R,(\alpha \beta), Q, u) \in \widehat{\mathscr{C}}_{g} \quad(n \geqq 0)$.

2) In the limit $(n \rightarrow \infty)$ we have a surjective linear mapping

$$
\theta_{X}: \operatorname{Der}(\hat{K}) \rightarrow T_{X} \hat{\mathscr{C}}_{g}
$$

If we denote the kernel of $\theta_{X}$ by $B(X)$, then it satisfies the following exact sequence:

$$
0 \rightarrow B(X) \rightarrow \bar{B}(X) \rightarrow H^{0}(R, \Theta) \rightarrow 0
$$

and we have a commutative diagram of short exact sequences:

$$
\begin{aligned}
& \begin{array}{ccc}
0 \longrightarrow T_{X} \hat{\mathscr{C}}_{g} / \mathscr{C}_{g} \longrightarrow T_{X} \hat{\mathscr{C}}_{g} & \longrightarrow \quad \tilde{\omega}^{*} T_{Q} \mathscr{C}_{g} & \longrightarrow 0 \\
\imath \downarrow & \downarrow \downarrow & \imath \downarrow
\end{array} \\
& 0 \longrightarrow \mathfrak{G}_{0}=\hat{\mathcal{O}} \zeta \frac{d}{d \zeta} \longrightarrow \frac{\operatorname{Der}(\hat{K})}{B(X)} \longrightarrow \frac{\operatorname{Der}(\hat{K})}{\hat{\hat{O}} \zeta \frac{d}{d \zeta}+B(X)} \longrightarrow 0,
\end{aligned}
$$

where $T \hat{\mathscr{C}}_{\mathrm{g}} / \mathscr{C}_{\mathrm{g}}$ is the set of tangent vectors along the fibre of $\tilde{\omega}$.

3) The above isomorphism defines a Lie anti-homomorphism

$$
\theta: \mathfrak{G}=\operatorname{Der}(\hat{K}) \rightarrow H^{0}\left(\hat{\mathscr{C}}_{g}, \Theta_{\hat{\mathscr{C}}_{g}}\right)
$$

whose restriction to $\mathfrak{5}_{0}$ coincides with the one induced from the action of $G_{0}$,

$$
\theta:\left(\mathfrak{5}_{0}=\hat{\mathcal{O}} \zeta \frac{d}{d \zeta} \rightarrow H^{0}\left(\hat{\mathscr{C}}_{g}, \Theta_{\hat{\mathscr{C}}_{g}}\right)\right.
$$


4) For $\forall X \in \hat{\mathscr{C}}_{g}$ the following diagram is commutative:

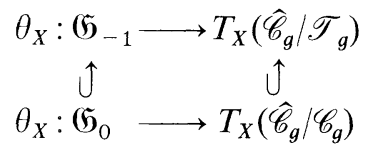

(2.20) Remark. For $\forall \ell \in \mathbb{G}, \theta(\ell)$ is a holomorphic vector field on $\hat{\mathscr{C}}$, and for $\forall X \in \hat{\mathscr{C}}_{g}, T_{X} \hat{\mathscr{C}}_{g}$ is spanned by $\left\{\theta_{X}(\ell), \ell \in \mathbb{G}\right\}$. In this sense we say that the triple $\left(\hat{\mathscr{C}}_{g},(\mathfrak{5}, \theta)\right.$ is an infinitesimally homogeneous space [B.R.].

\section{D) Formal Trivialization}

(2.21) We still consider the family of Riemann surfaces in (2.18). The union of Picard varieties of fibres forms a holomorphic family of abelian Lie groups:

$$
p: \mathscr{P}_{g}=\bigcup_{[R] \in \mathscr{T}_{g}} P(R) \rightarrow \mathscr{T}_{g}
$$

It decomposes into connected components as

$$
\mathscr{P}_{g}=\coprod_{d \in \mathbb{Z}} \mathscr{P}_{g}^{(d)}=\coprod_{x \in \mathbb{Z}} \mathscr{P}_{g}^{x} \quad[\text { cf. (2.16)] }
$$

The fibre product $\mathscr{X}_{g}=\mathscr{C}_{g} \underset{\mathscr{T}_{g}}{\times} \mathscr{P}_{g}$ is the set of triples $\{(R,(\alpha, \beta), Q, \mathscr{L})$ with $Q \in R$, $\mathscr{L} \in P(R)\}$ and by projection we have a commutative diagram

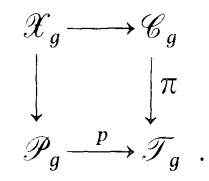

(2.22) Definition. 1) For each $\mathscr{L} \in \operatorname{Pic}(R)$ with $Q \in R$ we define

$$
\hat{\mathscr{L}}_{Q}=\lim _{n} \mathscr{L}_{Q} / m_{Q}^{n+1} \mathscr{L}_{Q},
$$

which is left $\hat{\mathcal{O}}_{Q}$-module free of rank 1.

2) The space $\hat{X}_{g}$ is a set of quintuples $\{(X, L)=(R,(\alpha, \beta), Q, \mathscr{L}, u, t) ; Q \in R$, $\left.\mathscr{L} \in P(R), u: \hat{\mathcal{O}}_{Q} \simeq \hat{\mathcal{O}}, t: \hat{\mathscr{L}}_{Q} \simeq \hat{\mathcal{O}}\right\}$, where $u$ is a formal uniformization (2.18) and $t$ is a $u$-linear isomorphism of modules (i.e. for $f \in \hat{\mathcal{O}}_{Q}$ and $s \in \hat{\mathscr{L}}_{Q}, t(f s)=u(f) t(s)$ ) called a formal trivialization or formal gauge fixing of $\mathscr{L}$ at $Q$. This $t$ is determined by the (formal) section $\hat{s}=t^{-1}(1)$ which is a (formal) generator of $\mathscr{L}$ at $Q$.

For a given data $(R, Q, \mathscr{L})$ with a Riemann surface $R, Q \in R$ and $\mathscr{L} \in P(R)$ we denote by $K_{\mathscr{L}}$ the $\mathbb{C}$-vector space of meromorphic sections of $\mathscr{L}$ which is a free $K=K(R)$-module of rank 1 . We further define $\mathscr{D}_{K}^{1}\left(K_{\mathscr{L}}\right)=\left\{D ; K_{\mathscr{L}} \rightarrow K_{\mathscr{L}}, \mathbb{C}\right.$-linear map such that $\exists \bar{D} \in \operatorname{Der}(K)$, for $\left.\forall f \in K, \forall \varphi \in K_{\mathscr{L}} ; D(f \varphi)=\bar{D}(f) \varphi+f D(\varphi)\right\}$. We then obtain the following exact sequence:

$$
0 \longrightarrow K \stackrel{i}{\longrightarrow} \mathscr{D}_{K}^{1}\left(K_{\mathscr{L}}\right) \stackrel{\pi}{\longrightarrow} \operatorname{Der}(K) \longrightarrow 0 \text {. }
$$

For $g \in K, i(g) \in \mathscr{D}_{K}^{1}\left(K_{\mathscr{L}}\right)$ is defined by $i(g)(\varphi)=g \varphi$ for $\forall \varphi \in K_{\mathscr{L}}$, and for $D \in \mathscr{D}_{K}^{1}\left(K_{\mathscr{L}}\right), \pi(D)=\bar{D}$. 
We next define the Lie subalgebras of $\mathscr{D}_{K}^{1}\left(K_{\mathscr{L}}\right)$ by

$$
\begin{aligned}
& \bar{B}(R, Q, \mathscr{L}) \equiv\left\{D \in \mathscr{D}_{K}^{1}\left(K_{\mathscr{L}}\right) \mid D: H^{0}(R, \mathscr{L}(* Q)) \rightarrow H^{0}(R, \mathscr{L}(* Q))\right\}, \\
& B(R, Q, \mathscr{L})=\pi^{-1}(B(R, Q)) .
\end{aligned}
$$

(2.23) Proposition. With the notations above we obtain the following commutative diagram of the short exact sequence:

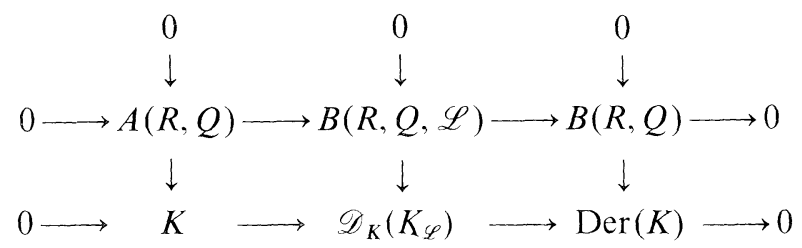

We further define $\hat{K}_{\mathscr{L}, Q} \equiv \underset{n}{\lim } K_{\mathscr{L}} / \mathrm{m}_{Q}^{n+1} \mathscr{L}_{Q}$, which is a free $\hat{K}_{Q}$-module of rank 1 . The following relations hold naturally:

$$
\hat{K}_{\mathscr{L}, Q}=K \underset{\mathscr{O}_{Q}}{\otimes} \hat{\mathscr{L}}_{Q}=\hat{K}_{Q} \underset{\mathscr{O}_{Q}}{\otimes} \mathscr{L}_{Q}
$$

We have natural projections $\varrho: \hat{\mathscr{X}}_{g} \rightarrow \mathscr{X}_{g}, \hat{\mathscr{X}}_{g} \rightarrow \hat{\mathscr{C}}_{g}$ which form a commutative diagram of holomorphic maps

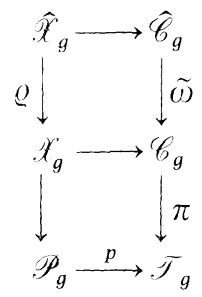

Like $\widehat{\mathscr{C}}_{g}$ this $\hat{\mathscr{X}}_{g}$ also is a projective limit of finite dimensional complex manifolds. $\hat{\mathscr{X}}_{g}$ is a disjoint union of connected components $\hat{\mathscr{X}}_{g}^{\chi}$ each of which lies over $\mathscr{P}_{g}^{\chi}$ respectively.

On $\hat{\mathscr{X}}_{g}$ the group $\widetilde{G}_{0},(2.6)$ acts (from the left) as $(u, t) \rightarrow(\varphi \circ u, \varphi(\sigma \cdot t))$ for $\varphi \in G_{0}$, $\sigma \in \hat{\mathcal{C}}^{*}$. With this action $\hat{\mathscr{X}}_{g}$ becomes a holomorphic principal homogeneous space over $\mathscr{X}_{g}$ with $\widetilde{G}_{0}$ as fibres.

(2.24) Definition. Denote by $\mathbb{P}\left(\hat{\mathscr{X}}_{g}\right)$ the projective space associated with $\hat{\mathscr{X}}_{g}$ on which $\mathbb{C}^{*}$ acts by $\mathbb{C}^{*} \subset \hat{\mathcal{O}}^{*}$. This is equivalent to considering the formal trivialization up to constants. We can take the quotient since $\mathbb{C}^{*}$ is contained in the centre of $\widetilde{G}_{0}$. This space is what we are mainly concerned with.

For each data $(X, L)=(R,(\alpha, \beta), Q, \mathscr{L}, u, t) \in \hat{X}_{g}, u$-linear $\left(u: \hat{\mathcal{O}}_{Q} \rightarrow \hat{\mathcal{O}}\right)$ isomorphism $t: \hat{\mathscr{L}}_{Q} \rightarrow \hat{\mathcal{O}}_{Q}$ induces $u$-linear $\left(u: \hat{K}_{Q} \stackrel{\sim}{\longrightarrow} \hat{K}\right)$ isomorphism $t: \hat{K}_{\mathscr{L}, Q} \rightarrow \hat{K}$. Then each element $D \in \mathscr{D}_{K}^{1}\left(K_{\mathscr{L}}\right)$ defines $t(D) \in \mathscr{D}^{1}(\hat{K})$. We then define a Lie subalgebra of $\mathscr{D}^{1}(\hat{K})$ by $B(X, L)=\{t(D) ; D \in B(R, Q, \mathscr{L})\}$. From Proposition (2.23) we obtain the following exact sequence:

$$
0 \longrightarrow A(X) \longrightarrow B(X, L) \longrightarrow B(X) \longrightarrow 0 \text {. }
$$


For $(X, L) \in \hat{\mathscr{X}}_{g}$ we denote the corresponding element in $\mathbb{P}\left(\hat{\mathscr{X}}_{g}\right)$ by $((X, L))$. Then in the same way as (2.19) we have the following.

Proposition. 1) There is an isomorphism

$$
T_{((X, L))} \mathbb{P}\left(\hat{\mathscr{X}}_{g}\right) \sim \mathscr{D}^{1}(\hat{K}) / B(X, L),
$$

which induces a commutative diagram of short exact sequences

$$
\begin{aligned}
& 0 \rightarrow T_{((X, L))} \mathbb{P}\left(\hat{\mathscr{X}}_{g}\right) / \mathscr{X}_{g} \rightarrow T_{((X, L))} \mathbb{P}\left(\hat{\mathscr{X}}_{g}\right) \rightarrow Q^{*} T_{(Q, \mathscr{L})} \mathscr{X}_{g} \rightarrow 0 \\
& 0 \rightarrow \quad \mathfrak{B}+\mathfrak{H}_{-} \longrightarrow \frac{\mathscr{D}^{1}(\hat{K})}{B(X, L)} \longrightarrow \frac{\mathscr{D}^{1}(\hat{K})}{B(X, L)+\mathfrak{B}+\mathfrak{U}_{-}} \rightarrow 0 .
\end{aligned}
$$

2) The above isomorphism defines a Lie anti-homomorphism

$$
\theta: \widetilde{\mathfrak{G}}=\mathscr{D}^{1}(\widehat{K}) \rightarrow H^{0}\left(\mathbb{P}\left(\hat{\mathscr{X}}_{g}\right), \Theta_{\mathbb{P}\left(\hat{\mathscr{X}}_{g}\right)}\right)
$$

whose restriction to $\widetilde{\mathfrak{G}}_{0}=\mathfrak{B}+\mathfrak{U}$ coincides with that induced from the action of $\widetilde{G}_{0}$ on $\hat{\mathscr{X}}_{g}$ :

$$
\theta: \widetilde{\mathfrak{G}}_{0} \longrightarrow H^{0}\left(\mathbb{P}\left(\hat{\mathscr{X}}_{g}\right), \Theta_{\mathbb{P}\left(\hat{\mathscr{x}}_{g}\right)}\right)
$$

(Again $\left(\mathbb{P}\left(\hat{\mathscr{X}}_{g}, \widetilde{\mathfrak{G}}_{0}, \theta\right)\right.$ is an infinitesimal homogeneous space in the sense of Remark (2.20).)

\section{E) Embedding of Weierstrass System Into UGM}

(2.26) Recall that $M=\operatorname{Sp}(2 g, \mathbb{Z})$ acts on the Torelli space $\mathscr{T}_{g}(2.18)$. It is easy to see from the construction that the action of $M$ lifts to that on $\hat{\mathscr{X}}_{g}$ etc. and is compatible with the diagram in (2.23). We can take the quotient $\hat{\mathscr{M}}_{g}=\mathbb{P}\left(\hat{\mathscr{X}}_{g}\right) / M$. This moduli space $\hat{\mathscr{M}}_{g}$ of framed and gauged Riemann surfaces is called the Weierstrass system (of genus $g$ ).

(2.27) We define a map

$$
\Gamma: \mathbb{P}(\hat{X}) \longrightarrow \operatorname{UGM}(\hat{K})
$$

sending $(X, L)=(R,(\alpha, \beta), Q, \mathscr{L}, u, t)$ to $t\left(H^{0}(R, \mathscr{L}(* Q))\right) \subset \hat{K}$, which is seen to be well-defined and holomorphic. In other words the image is the set of Laurent series expansions of meromorphic sections of $\mathscr{L}$ holomorphic outside $Q$ by means of the chosen (formal) coordinates and gauge. $\Gamma$ can be regarded as the period map of $\mathbb{P}\left(\hat{\mathscr{X}}_{g}\right)$. Note that the period domain UGM does not depend on the genus $g$.

We have the main theorem in this section, which corresponds to Torelli's theorem for the Weierstrass system.

(2.28) Theorem. 1) $\Gamma$ maps $\mathbb{P}\left(\hat{\mathscr{X}}^{\chi}\right)$ into $\mathrm{UGM}^{\chi}$.

2) The value of $\Gamma$ does not change under the action of $M$, hence we have a holomorphic map

$$
\bar{\Gamma}: \hat{\mathscr{M}}_{g}=\mathbb{P}\left(\hat{\mathscr{X}}_{g}\right) / M \longrightarrow \operatorname{UGM}(\hat{K}) .
$$


3) $\bar{\Gamma}$ is injective for $g>1$ (for $g \leqq 1$, replace $M$ by $M \times \operatorname{Aut}(R)$ ). Namely $U=t\left(H^{0}(R, \mathscr{L}(* Q)) \subset \hat{K}\right.$ determines the data $(X, L)$ up to isomorphisms and scalar.

Proof. Only the last statement 3 ) is non-trivial. We have however

and

$$
K=K(R)=\{f / g \in \hat{K} ; f, g \in U\}
$$

$$
A(R, Q)=\{h \in K ; h U \subset U\} .
$$

Hence $R$ is determined by $K$ and $Q$ by $A(R, Q)$, since we have

$$
\begin{gathered}
\mathcal{O}_{Q}=\left\{f / g ; f, g \in A(R, Q), v_{Q}(f) \geqq v_{Q}(g)\right\}, \\
\left(v_{Q}(f)=\max \left\{n ; f \in F^{n}\right\}\right) .
\end{gathered}
$$

On the other hand, as $R-\{Q\}$ is affine, sections in $H^{0}(\mathscr{L}(* Q))$ generates $\mathscr{L}_{Q^{\prime}}, Q^{\prime} \neq Q$ and $\mathscr{L}_{Q}$ is characterized as

$$
\left\{f \in H^{0}(\mathscr{L}(* Q)) \underset{A(R, Q)}{\otimes} K ; v_{Q}(f) \geqq 0\right\}
$$

(considered as $\mathscr{L} \subset \hat{K}_{\mathscr{L}}$ (constant sheaf)). Since $A$ and $U$ are already contained in $\hat{K}$, the formal uniformization and the local trivialization are also uniquely determined (up to scalar).

(2.29) Theorem. For each pair $(X, L) \in \widehat{\mathscr{X}}_{g}$ and $((X, L)) \in \mathbb{P}\left(\hat{\mathscr{X}}_{g}\right)$,

1) the following diagram is commutative;

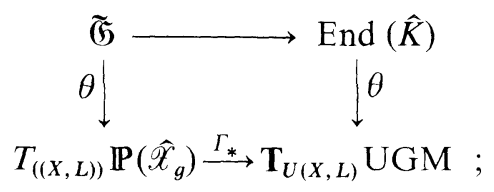

2) for each $\widetilde{\mathfrak{G}} D$, the holomorphic vector field $\theta(D)$ on $\hat{\mathscr{X}}_{g}$ is modular invariant,

$$
\gamma_{*} \theta_{(X, L)}(D)=\theta_{\gamma(X, L)}(D) \quad \text { for } \quad \gamma \in M \text {; }
$$

3) (the local Torelli theorem) the map

$$
\Gamma_{*} ; T_{((X, L))} \mathbb{P}(\hat{\mathscr{X}}) \rightarrow T_{U(X, L)} \mathrm{UGM}
$$

is injective if $g>1$ (in general $\operatorname{Ker} \Gamma_{*}=\bar{B}(X, L) / B(X, L)$ ).

Proof. 1) and 2) are trivial from the definition of $\theta$. We prove 3). From Proposition (2.25) we have a natural identification: $T_{((X, L))} \mathbb{P}(\hat{\mathscr{X}}) \cong \mathscr{D}^{1}(K) / B(X, L)$. For a given $D \in \mathscr{D}^{1}(\hat{K})$ the element

$$
\Gamma_{*}(D) \in T_{U(X, L)} \mathrm{UGM} \equiv \operatorname{Hom}_{\mathbb{C}}(U(X, L), \hat{K} / U(X, L))
$$

is represented by the map

$$
\Gamma_{*}(D): U(X, L) \longrightarrow \hat{K} \stackrel{D}{\longrightarrow} \hat{K} \longrightarrow \hat{K} / U(X, L) .
$$

If we assume $\Gamma_{*}(D)=0$, then we recognize that $D: H^{0}(R, \mathscr{L}(* Q))$ $\rightarrow H^{0}(R, \mathscr{L}(* Q))$, since $U(X, L)=t H^{0}(R, \mathscr{L}(* Q))$, where we have omitted the 
symbol $t$. On the other hand $K=K(R)$ is generated by $\left\{\psi / \varphi ; \psi, \varphi \in H^{0}(R, \mathscr{L}(* Q))\right.$, $\varphi \neq 0\}$ as a $\mathbb{C}$-algebra. Considering $\bar{D}=\pi(D) \in \operatorname{Der}(\widehat{K})$, we obtain:

$$
\bar{D}(\psi / \varphi)=\frac{(D \psi) \varphi-\psi D(\varphi)}{\varphi^{2}} \text { for } \varphi, \psi \in H^{0}(R, \mathscr{L}(* Q)) .
$$

Since $D(\varphi), D(\psi) \in H^{0}(R, \mathscr{L}(* Q)), \bar{D}$ belongs to $\operatorname{Der}(K)$. On the other hand $D$ preserves $H^{0}(R, \mathscr{L}(* Q))$ and $K_{\mathscr{L}}=K \underset{A(R, Q)}{\otimes} H^{0}(R, \mathscr{L}(* Q))$, hence we have $D: K_{\mathscr{L}} \rightarrow K_{\mathscr{L}}$, in other words $D \in \mathscr{D}_{K}^{1}\left(K_{\mathscr{L}}\right)$. From the condition $D: H^{0}(R, \mathscr{L}(* Q))$ $\rightarrow H^{0}(R, \mathscr{L}(* Q))$ we reach the result $D \in B(X)$.

\section{F) Sections of Spin}

(2.30) For $j \in \mathbb{Z}$ we have a section $\sigma_{j}: \mathscr{T}_{g} \rightarrow \mathscr{P}_{g}$ sending $[R]$ to $\left([R], \Omega_{R}^{\otimes j}\right)$, where $\Omega_{R}$ is the canonical sheaf (or the sheaf of holomorphic forms on $R$ ). Note that the degree of $\Omega_{R}^{\otimes j}$ is equal to $2(g-1) j$, hence the Euler-characteristic is $(2 j-1)(g-1)$.

This section lifts to $\hat{\sigma}_{j}: \hat{\mathscr{C}}_{g} \rightarrow \hat{\mathscr{X}}_{g}$ (called of spin $j$ ) since $u: \hat{\mathscr{O}}_{Q} \rightarrow \hat{\mathcal{O}}$ induces $(d u)^{j}: \hat{\Omega}_{R, Q}^{\otimes j} \rightarrow \hat{\mathcal{O}}$ sending $f\left(d u^{-1}(\zeta)\right)^{j}$ to $u(f)$.

(2.31) Now we want to define such section for $j \in \frac{1}{2} \mathbb{Z}$. Using the theory of Riemann constant for each $(R,(\alpha, \beta)) \in \mathscr{T}_{g}$, we can canonically associate a line bundle $\mathscr{L}_{\Delta}(R,(\alpha, \beta))=\mathscr{L}_{\Delta}$ such that $\mathscr{L}_{\Delta}^{2}=\Omega_{R}$ [B.M.; A-G.N.V.]. Here we define $\sigma_{j} ; \mathscr{T}_{g} \rightarrow \mathscr{P}_{g}^{(2 j-1)(g-1)}$, as $\sigma_{j}(R,(\alpha, \beta)) \equiv\left(R,(\alpha, \beta), \mathscr{L}_{\Delta}^{2 j}\right)$. We define the following formal trivialization $\sqrt{d u} ; \hat{\mathscr{L}}_{\Delta, Q} \stackrel{\sim}{\longrightarrow} \hat{\mathcal{O}}_{Q}$ with $(\sqrt{d u})^{2}=d u:\left(\hat{\mathscr{L}}_{\Delta, Q}\right)^{2}=\hat{\Omega}_{Q} \stackrel{\sim}{\longrightarrow} \hat{\mathcal{O}}$, which is determined up to constants \pm 1 .

For $j \in \frac{1}{2} \mathbb{Z}$ with an identification $\Omega_{k}^{j}=\mathscr{L}_{\Delta}^{2 j}$, we define a mapping $\hat{\sigma}_{j} ; \hat{\mathscr{C}}_{g} \rightarrow \mathbb{P}\left(\hat{\mathscr{X}}_{g}^{(2 j-1)(g-1)}\right)$, by

$$
\hat{\sigma}_{j}((R,(\alpha, \beta), Q, u)) \equiv\left(R,(\alpha, \beta), Q, u, \mathscr{L}_{\Delta}^{2 j},(d u)^{j}\right),
$$

where $(\sqrt{d u})^{2 j} ; \hat{\mathscr{L}}_{\Delta, Q}^{2 j} \cong\left(\hat{\mathscr{L}}_{\Delta, Q}\right)^{\otimes 2 j} \stackrel{\sim}{\longrightarrow} \widehat{\mathcal{O}}$.

Then we have a commutative diagram

$$
\begin{aligned}
\hat{\sigma}_{j}: \hat{\mathscr{C}}_{g} & \rightarrow \mathbb{P}\left(\hat{\mathscr{X}}_{g}^{(2 j-1)(g-1)}\right) \\
\downarrow & \downarrow \\
\sigma_{j}: \mathscr{T}_{g} & \rightarrow \mathscr{P}_{g^{\prime}}^{(2 j-1)(g-1)} .
\end{aligned}
$$

We define the following composite holomorphic map

$$
\Gamma \circ \hat{\sigma}_{j}: \hat{\mathscr{C}}_{g} \rightarrow \mathrm{UGM}^{(2 j-1)(g-1)},
$$

which we call the modular embedding of spin $j$.

(2.32) Proposition. For $j \in \frac{1}{2} \mathbb{Z}$ and a section

$$
\begin{aligned}
& s_{j}: \operatorname{Der}(\hat{K}) \longrightarrow \mathscr{D}^{1}(\hat{K}) \\
& \text { (1) U } \\
& f(\zeta) \frac{d}{d \zeta} \mapsto f(\zeta) \frac{d}{d \zeta}+j \frac{d f}{d \zeta}(\zeta),
\end{aligned}
$$


we have a commutative diagram

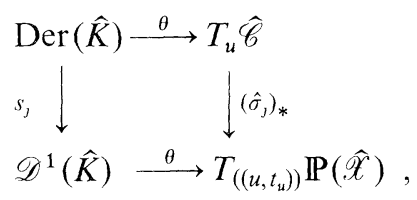

where horizontal arrows are defined in (2.19), (2.25).

We are mainly interested in the case $j=\frac{1}{2}$. For simplicity we denote $\hat{\sigma}_{1 / 2} ; \hat{\mathscr{C}}_{g} \rightarrow \mathbb{P}\left(\hat{X}^{0}\right)$ by $\hat{\sigma}$.

(2.33) By using the period matrix $\Omega$, the jacobian variety $J$ of $R$ can be expressed as the quotient of $\mathbb{C}^{g}$ by a lattice $L=\left(1_{g}, \Omega\right) \mathbb{Z}^{2 g}$ of rank $2 g$ through the identification of $\widetilde{J}=\operatorname{Hom}\left(H^{0}\left(R, \Omega_{R}\right), \mathbb{C}\right)$ with $\mathbb{C}^{g}$. For an element $c={ }^{t}\left(c_{1}, \ldots, c_{g}\right) \in \mathbb{C}^{g}$, the corresponding element $c \in \widetilde{J}$ satisfies $c\left(\omega_{i}\right)=c_{i}$. Choosing a point $Q \in R$, we have an embedding $I_{Q}: R \rightarrow J, P \mapsto\left(\int_{Q}^{P} \omega^{i}\right) \bmod L$.

(2.34) For $c \in \widetilde{J}\left(=\mathbb{C}^{g}\right)$ denote by $\mathscr{L}_{c}$ the line bundle of degree 0 on $R$ corresponding to $c \bmod L$ in $J$. The section of $\mathscr{L}_{c}$ can be identified with the space of multiplicative functions on $R$,

$$
\left\{f: \widetilde{R} \rightarrow \mathbb{C} ; f\left(\tilde{p}+{ }^{t} m \alpha+{ }^{t} n \beta\right)=\exp \left(2 \pi \sqrt{-1^{t}} n c\right) f(\tilde{p}), m, n \in \mathbb{Z}^{g}\right\},
$$

where $\widetilde{R}$ is the maximal abelian covering of $R$. Moreover a multiplicative function on $\widetilde{R}$ can be considered as a multivalued meromorphic function on $R$. This implies that for $Q \in R$ and $c \in \widetilde{J}$ we have chosen a local trivialization $s_{c}:\left(\hat{\mathscr{L}}_{c}\right)_{Q} \simeq \hat{\mathcal{O}}_{Q}$ via this identification.

Denote by $\tilde{\mathscr{I}}_{g}\left(=\left(\pi_{*} \Omega_{\mathscr{C} / \mathscr{T}}\right)^{\vee}\right)$ the family of universal coverings of $J(R)$ 's, which is a vector bundle on $\mathscr{T}_{g}$ of rank $g$. The dual basis of $\left\{\omega_{i}\right\}$ gives the trivialization of vector bundle $\pi: \tilde{\mathscr{I}}_{g} \rightarrow \mathscr{T}_{g}$;

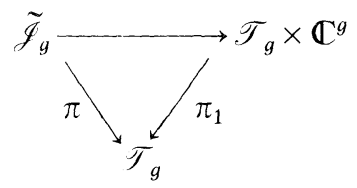

We set $\hat{\mathscr{C}}_{g}(\tilde{\mathscr{I}}) \equiv \hat{\mathscr{C}}_{g} \times \tilde{\mathscr{I}}=a$ set of data $\left(R,(\alpha, \beta), Q, u, \mathscr{L}_{c}\right)$ and define a map $\tilde{\sigma}_{1 / 2}: \hat{\mathscr{C}}_{g}(\tilde{\mathscr{I}}) \rightarrow \mathbb{P}\left(\hat{\mathscr{X}}^{(0)}\right)^{\mathscr{T}_{g}}$ by

$$
\tilde{\sigma}=\tilde{\sigma}_{1 / 2}:\left(R,(\alpha, \beta), Q, u, \mathscr{L}_{c}\right) \rightarrow\left(R, Q, \mathscr{L}_{\Delta} \otimes \mathscr{L}_{c}, u, \pm \sqrt{d u} \otimes\left(u \circ s_{c}\right)\right)
$$

with $s_{c}$ defined above.

(2.35) Proposition. 1) The following diagram is commutative

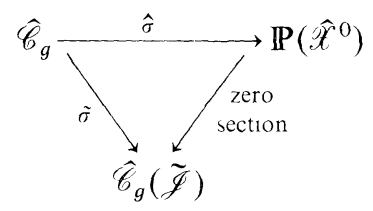


2) The following action of vector fields are compatible:

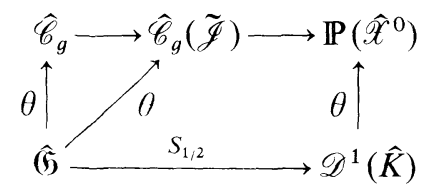

where $\theta(\mathfrak{G})$ acts on $\hat{\mathscr{C}}_{g}(\tilde{\mathscr{I}})=\hat{\mathscr{C}}_{g} \underset{\mathscr{T}_{g}}{\times} \tilde{\mathscr{I}}$ to its first component only.

(2.36) Define the actions of $\mathbb{Z}^{2 g}$ and the modular group $M$ on $\hat{\mathscr{C}}(\tilde{\mathscr{I}})$ as follows:

1) $\mathbb{Z}^{2 g}(m, n)$ acts on $\hat{\mathscr{C}}_{g}(\tilde{\mathscr{I}}) \rightarrow \hat{\mathscr{C}}_{g}$ as a fibrewise transformation by $c \rightarrow c+\Omega m+n$.

2) The modular group $M$ acts on $\hat{\mathscr{C}}_{g}(\tilde{\mathscr{I}})$ as

$$
\gamma(X, c)=\left(\gamma(X),{ }^{t}(C \Omega+D)^{-1} c\right) \text { for } \quad \gamma=\left(\begin{array}{ll}
A & B \\
C & D
\end{array}\right) \in M \text {. }
$$

(2.37) Proposition. With the above notations, the map $\tilde{\sigma}_{1 / 2}: \hat{\mathscr{C}}_{g}(\tilde{\mathscr{I}}) \rightarrow \mathbb{P}\left(\hat{\mathscr{X}}_{g}^{0}\right)$ is invariant under the action of $\tilde{M}_{\Delta}=M_{\Delta} \ltimes \mathbb{Z}^{2 g}$, where $M_{\Delta}(=M \cap \Gamma(2,4)) \subset M$ is the subgroup of transformations which preserve the Riemann constant $\Delta$ (cf. Sect. 5, C)).

\section{Fermion Fock Space and the Second Quantization}

(3.0) The main object of this section is to extend and lift the infinitesimal action of $\operatorname{End}(\mathscr{V})$ on UGM (as holomorphic vector fields on it) to the whole diagram

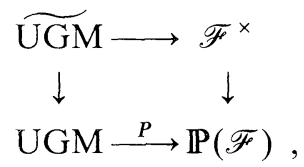

where $P$ is the Plücker embedding (1.31).

Here we encounter the difficulties of divergences coming essentially from the infinity of the dimension of UGM. We use a procedure of regularization (a technique of normal ordering, cf. $C$ )). In fact what acts on $\mathscr{F}$ is not End( $\mathscr{V}$ ) itself but its non-trivial extension by a 1 -dimensional center. This phenomenon is what physicists call anomaly. The Virasoro algebra and the current algebra appear as fundamental subalgebras of this extension (cf. $D)$ ).

\section{A) Fermion Operators}

(3.1) Definition. i) The associative algebra with 1 generated by $\psi_{\mu}, \bar{\psi}_{\mu}, \mu \in \mathbb{Z} h$, with the relations:

$$
\begin{aligned}
& {\left[\psi_{\mu}, \psi_{v}\right]_{+}=\left[\bar{\psi}_{\mu}, \bar{\psi}_{v}\right]_{+}=0, \quad\left[\psi_{\mu}, \bar{\psi}_{v}\right]_{+}=\delta_{\mu+v, 0}} \\
& \text { for } \forall \mu, v \in \mathbb{Z} h, \quad \text { where }[A, B]_{+}=A B+B A ;
\end{aligned}
$$

(i.e. the Clifford algebra) is called the fermion operator algebra, which we denote by $\mathscr{A}$. 
We define actions of $\psi_{\mu}, \bar{\psi}_{\mu}\left(=\bar{\psi}^{-\mu}\right)$ on $\mathscr{F}$ and $\overline{\mathscr{F}}$ as follows :

$$
\begin{array}{ll}
\left.\psi_{\mu}=\frac{\vec{\partial}}{\partial e^{\mu}}, \quad \bar{\psi}^{\mu}=e^{\mu} \wedge \quad \text { (left action on } \mathscr{F}\right), \\
\left.\psi_{\mu}=\wedge \bar{e}_{\mu}, \quad \bar{\psi}^{\mu}=\frac{\delta}{\partial \bar{e}_{\mu}} \quad \text { (right action on } \overline{\mathscr{F}}\right) .
\end{array}
$$

Then the above anti-commutation relations hold, i.e. $\mathscr{A}$ acts on $\mathscr{F}$ and $\overline{\mathscr{F}}$.

We denote by $W$ (respectively $\bar{W}$ ) the vector space generated by $\left\{\psi_{\mu}\right\}$ (respectively $\left.\left\{\bar{\psi}_{\mu}\right\}\right)(\mu \in \mathbb{Z} h)$ and set $\tilde{V}=W \oplus \bar{W}$.

(3.2) Proposition. These operators satisfy the following relations:

1) for $\forall\langle u|\in \overline{\mathscr{F}}| v,\rangle \in \mathscr{F}, \mu \in \mathbb{Z} h$,

$$
\begin{aligned}
& \langle u|\left(\psi_{\mu}|v\rangle\right)=\left(\langle u| \psi_{\mu}\right)|v\rangle, \\
& \langle u|\left(\bar{\psi}_{\mu}|v\rangle\right)=\left(\langle u| \bar{\psi}_{\mu}\right)|v\rangle ;
\end{aligned}
$$

2) for $\forall p \in \mathbb{Z}$,

$$
\begin{aligned}
& \psi_{\mu}|p\rangle=0(\mu>p), \quad\langle p| \psi_{\mu}=0(\mu<p), \\
& \bar{\psi}_{\mu}|p\rangle=0(\mu>-p), \quad\langle p| \bar{\psi}_{\mu}=0(\mu<-p),
\end{aligned}
$$

where $|p\rangle,\langle p|$ are charged vacua defined in (1.25).

Definition. We set

$$
\begin{array}{lll}
W=W_{+} \oplus W_{-}, & W_{+}=\underset{\mu>0}{\oplus} \mathbb{C} \psi_{\mu}, & W_{-}=\underset{\mu<0}{\oplus} \mathbb{C} \psi_{\mu}, \\
\bar{W}=\bar{W}_{+} \oplus \bar{W}_{-}, \quad \bar{W}_{+}=\underset{\mu>0}{\oplus} \mathbb{C} \bar{\psi}_{\mu}, & \bar{W}_{-}=\underset{\mu<0}{\oplus} \mathbb{C} \bar{\psi}_{\mu}, \\
\tilde{V}_{+}=\{\psi \in \tilde{V} ; \psi|0\rangle=0\}=W_{+} \oplus \bar{W}_{+}, \\
\tilde{V}_{-}=\{\psi \in \tilde{V} ;\langle 0| \psi=0\}=W_{-} \oplus \bar{W}_{-} .
\end{array}
$$

Elements of $\tilde{V}_{+}$(respectively $\tilde{V}_{-}$) are called annihilation operators (respectively creation operators).

(3.4) In Sect. 1 the Fock space was defined as a semi-infinite exterior product (geometric interpretation). There is, however, another algebraic presentation of the Fock space by means of the operator algebra $\mathscr{A}$. This form is more convenient for concrete calculations.

(3.5) Theorem. 1. The following homomorphism of left $\mathscr{A}$-modules is injective and its image is dense:

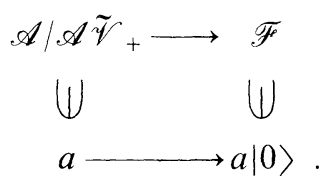


2) The following homomorphism of right $\mathscr{A}$-modules is isomorphic:

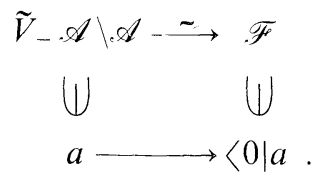

(3.6) The notion of "normal ordering," familiar to physicists but not to mathematicians, is one of the most important techniques in operator calculus. It can be formulated mathematically as follows:

We define a linear map (called normal ordering)

$$
: \quad ; \quad \wedge \tilde{V} \longrightarrow \wedge\left(\tilde{V}_{-}\right) \otimes \wedge\left(\tilde{V}_{+}\right) \stackrel{\sim}{\longrightarrow} \mathscr{A}
$$

such that i) it is an isomorphism as left $\wedge\left(\tilde{V}_{-}\right)$, right $\wedge\left(\tilde{V}_{+}\right)$-bimodule, and ii) $: 1:=1$. Such a linear map exists uniquely.

(3.7) Definition. We define the following fermion field operators

$$
\begin{aligned}
& \psi(z)=\sum_{\mu \in \mathbb{Z} h} \psi_{\mu} z^{-\mu-1 / 2}, \\
& \bar{\psi}(z)=\sum_{\mu \in \mathbb{Z} h} \bar{\psi}_{\mu} z^{-\mu-1 / 2},
\end{aligned}
$$

which are operator-valued infinite formal Laurent series.

(3.8) Proposition. In the region $|z|>|w|$ the vacuum expectation value of two operators are well defined;

$$
\begin{aligned}
& \psi(z) \bar{\psi}(w) \equiv\langle 0|\psi(z) \bar{\psi}(w)| 0\rangle=\frac{1}{z-w}, \\
& \overline{\psi(z) \psi(w)} \equiv\langle 0|\bar{\psi}(z) \psi(w)| 0\rangle=\frac{1}{z-w}, \\
& \psi(z) \psi(w) \equiv \bar{\psi}(z) \bar{\psi}(w)=0 .
\end{aligned}
$$

We extend the defining domain of $\bar{\psi}(z) \psi(w)$ from $|z|>|w|$ to $\left(\mathbb{C}^{*}\right)^{2}-\Delta$ $=\left\{(z, w) \in \mathbb{C}^{*} \times \mathbb{C}^{*} ; z \neq w\right\}$ by analytic continuation. The vacuum expectation value of general field operators $\langle 0|\ldots| 0\rangle$ can be defined in a similar way [T.K.].

Physicists know the following very convenient formula for calculations of fermion operator algebra.

(3.9) Theorem (Wick's theorem).

$$
\begin{aligned}
\phi\left(z_{1}\right) \ldots \phi\left(z_{N}\right) & =\sum_{\text {comb. }}: \phi\left(z_{1}\right) \ldots \phi\left(z_{1}\right) \ldots \phi\left(z_{j}\right) \ldots \phi\left(z_{k}\right) \ldots \phi\left(z_{v}\right) \ldots \phi\left(z_{N}\right): \\
& =\sum_{\text {comb. }}( \pm) \underset{\text { pars }}{\prod_{\phi\left(z_{i}\right) \phi}}\left(z_{j}\right): \prod_{\text {rest }} \phi\left(z_{k}\right):
\end{aligned}
$$

where $\phi(z)=\psi(z)$ or $\bar{\psi}(z)$. The summation $\sum_{\text {comb. }}$ is taken over all pairs in 
$\left\{z_{1}, z_{2}, \ldots, z_{N}\right\}$ and the sign \pm comes from the anticommuting relation of fermion operators (i.e. the signature of a permutation of indices).

Definition. 1) Define the filtration on $W$ and $\bar{W}$ as

$$
\begin{aligned}
& F^{m} W=\sum_{\mu>m} \mathbb{C} \psi^{\mu}=\sum_{\mu<-m} \mathbb{C} \psi_{\mu}, \\
& F^{m} \bar{W}=\sum_{\mu>m} \mathbb{C} \bar{\psi}^{\mu}=\sum_{\mu<-m} \mathbb{C} \bar{\psi}_{\mu},
\end{aligned}
$$

and introduce a topology on $W$ by this filtration.

2) Denote by $\mathscr{W}$ (respectively $\overline{\mathscr{W}}$ ) the completion of $W$ (respectively $\bar{W}$ ) with respect to this topology. Then we have

$$
\begin{aligned}
& \mathscr{W}=\mathscr{W}_{+} \oplus \mathscr{W}_{-}=\left(\underset{\mu>0}{\hat{\oplus}} \mathbb{C} \psi^{\mu}\right) \oplus\left(\underset{\mu<0}{\oplus} \mathbb{C} \psi^{\mu}\right), \\
& \overline{\mathscr{W}}=\overline{\mathscr{W}}_{+} \oplus \mathscr{W}_{-}=\left(\underset{\mu>0}{\hat{\oplus}} \mathbb{C} \bar{\psi}^{\mu}\right) \oplus\left(\underset{\mu<0}{\oplus} \mathbb{C} \bar{\psi}^{\mu}\right) .
\end{aligned}
$$

(3.11) Proposition. 1) There are filtration-preserving linear isomorphisms:

$$
\begin{array}{ll}
\overline{\mathscr{V}} \stackrel{\sim}{\longrightarrow} ; & \bar{e}_{\mu} \longrightarrow \psi_{\mu}, \\
\mathscr{V} \stackrel{\sim}{\longrightarrow} \overline{\mathscr{V}} ; & e^{\mu} \longrightarrow \bar{\psi}^{\mu} .
\end{array}
$$

2) The bilinear maps

$$
\begin{array}{ll}
\mathscr{W} \times \mathscr{F} \rightarrow \mathscr{F} ; & (\psi,|u\rangle) \mapsto \psi|u\rangle, \\
\overline{\mathscr{W}} \times \mathscr{F} \rightarrow \mathscr{F} ; & (\bar{\psi},|u\rangle) \mapsto \bar{\psi}|u\rangle
\end{array}
$$

are continuous on each variable.

(3.12) For a point $U \in \mathrm{UGM}$, we define $\bar{U} \subset \overline{\mathscr{V}}$ to be $U^{\perp}=\{f \in \operatorname{Hom}(\mathscr{V}, \mathbb{C})$; $\left.f_{\mid U}=0\right\}$, and we use the symbol [U] for the image of $U$ in $\mathbb{P}(\mathscr{F})$. The isomorphisms in (3.11) 1) define subspaces $\mathscr{W}_{+}(U) \subset \mathscr{W}$ and $\overline{\mathscr{W}}_{+}(U) \subset \overline{\mathscr{W}}$ corresponding to $\bar{U} \subset \overline{\mathscr{V}}$ and $U \subset \mathscr{V}$ respectively. The following theorem is very important (4.16).

(3.13) Theorem. We obtain the following equalities:

1) $\mathscr{W}_{+}(U)=\{\psi \in \mathscr{W} ; \psi|u\rangle=0,|u\rangle \in[U]\}$, $\overline{\mathscr{W}}_{+}(U)=\{\bar{\psi} \in \overline{\mathscr{W}} ; \bar{\psi}|u\rangle=0,|u\rangle \in[U]\}$.

2) $[U]=\left\{|u\rangle \in \mathscr{F} ; \psi|u\rangle=\bar{\psi}|u\rangle=0\right.$ for $\forall \psi \in \mathscr{W}_{+}(U)$, and $\left.\forall \bar{\psi} \in \overline{\mathscr{W}}_{+}(U)\right\}$.

We call $W_{+}(U) \oplus \bar{W}_{+}(U)$ as a space of annihilation operators associated with $U \in U G M$.

\section{B) A Differential Operator Algebra $\mathscr{D}_{z}$}

(3.14) Among endomorphisms of $\hat{K}=\mathbb{C}\left(\left(z^{-1}\right)\right)$, we consider such differential operators which form a subalgebra $\mathscr{D}_{z}$ of $\operatorname{End}(\hat{K})$, big enough and easy to treat. This 
restriction is only in order to make concrete calculation easier, and all the results in what follows can be generalized to the whole End $(\hat{K})$ in fact. The reader who knows the theory of KP equations could remark that this $\mathscr{D}_{z}$ is nothing but the "Fourier transform" of the algebra of microlocal differential operators $\mathscr{E}=\mathbb{C}[[t]]\left(\left(\partial^{-1}\right)\right)$.

(3.15) Definition. We consider the following differential operators:

$$
\mathscr{D}_{z}=\left\{P\left(z, \frac{d}{d z}\right)=\sum_{n \ll \infty} z^{n} a_{n}\left(\frac{d}{d z}\right) ; \quad a_{n}\left(\frac{d}{d z}\right) \in \mathbb{C}\left[\left[\frac{d}{d z}\right]\right]\right\} .
$$

We then define a valuation: $\operatorname{ord}_{z}: \mathscr{D}_{z} \rightarrow \mathbb{Z} \cup\{\infty\}$ in the following way;

$$
\operatorname{ord}_{z} P= \begin{cases}-m & \text { if } \quad P=\sum_{n \leqq m} z^{n} a_{n}\left(\frac{d}{d z}\right), \quad a_{m} \neq 0 \\ \infty & \text { if } \quad P=0\end{cases}
$$

(i.e. $\left.\operatorname{ord}_{z} z=-1, \operatorname{ord}_{z} \frac{\mathrm{d}}{\mathrm{dz}}=0\right)$. Define a (decreasing) filtration on $\mathscr{D}_{z}$ by

$$
G^{m} \mathscr{D}_{z}=\left\{P\left(z, \frac{d}{d z}\right) \in \mathscr{D}_{z} ; \operatorname{ord}_{z} P \geqq m\right\} .
$$

If we introduce a topology on $\mathscr{D}_{z}$ with this filtration, then $\mathscr{D}_{z}$ is complete Hausdorff and the product $\mathscr{D}_{z} \times \mathscr{D}_{z} \rightarrow \mathscr{D}_{z}$ is continuous (i.e. $\mathscr{D}_{z}$ is a complete topological $\mathbb{C}$-algebra) with respect to this topology.

(3.16) Proposition. 1) In case $\operatorname{ord}_{z} P=m$ for $P \in \mathscr{D}_{z}$, we have

$$
P: F^{\ell} \hat{K} \longrightarrow F^{\ell+m} \hat{K} \text {. }
$$

2) The natural map (defined from 1)) $\mathscr{D}_{z} \rightarrow \operatorname{End}(\hat{K})$ is injective (and $G^{m} \mathscr{D}_{z} \subset F^{m} \operatorname{End}(\hat{K})$ from 1$)$ ).

(3.17) One can rewrite $P\left(z, \frac{d}{d z}\right)$ as follows:

$$
P\left(z, \frac{d}{d z}\right)=\sum_{n \leqq m} z^{n} a_{n}\left(\frac{d}{d z}\right)=\sum_{k \leqq m} z^{k} g_{k}\left(z \frac{d}{d z}\right),
$$

where $g_{k}$ is a polynomial of order at most $m-k$ with respect to $z \frac{d}{d z}$. With this representation $\operatorname{ord}_{z}(P)$ is given by

$$
\operatorname{ord}_{z}(P)=-\min \left\{m: \operatorname{deg} g_{k} \leqq m-k \text { for } \forall k \in \mathbb{Z}\right\}
$$

Now we consider another valuation defined as

$$
v(P)=-\max \left\{k ; g_{k} \neq 0\right\}
$$

$\left(\right.$ i.e. $\left.v(z)=-1, v\left(z \frac{d}{d z}\right)=0\right)$. Clearly we have

$$
\operatorname{ord}_{z}(P) \leqq v(P)
$$


(3.18) Proposition. The filtration on $\mathscr{D}_{z}$ defined from $v, F^{m} \mathscr{D}_{z}=\left\{P \in \mathscr{D}_{z} ; v(P) \geqq m\right\}$, is nothing but the one induced from the inclusion map $\mathscr{D}_{z} \subset \operatorname{End}(\hat{K})$.

Proposition (3.16) implies that if a series in $\mathscr{D}_{z}$ converges with respect to $\operatorname{ord}_{z}$, then it converges with respect to $v$, but the converse need not hold.

\section{C) Second Quantization and Anomaly}

(3.19) Definition. For $P \in \mathscr{D}_{z}$ we define the adjoint $P^{\dagger} \in \mathscr{D}_{z}$ as follows:

1) $z^{\dagger}=z,\left(\frac{d}{d z}\right)^{\dagger}=-\frac{d}{d z}$

2) $(P Q)^{\dagger}=Q^{\dagger} P^{\dagger}$ for $\forall P, Q \in \mathscr{D}_{z}$ (anti-homomorphism).

A differential operator $P=P\left(z, \frac{d}{d z}\right) \in \mathscr{D}_{z}$ acts naturally on the formal parameter $z$ of the field operators $\psi(z), \bar{\psi}(z)$.

For $P \in \mathscr{D}_{z}, P$ and $P^{\dagger}$ are related as follows:

$$
\int_{\infty} d z:(P \psi(z)) \bar{\psi}(z):=\int_{\infty} d z: \psi(z)\left(P^{\dagger} \bar{\psi}(z)\right):,
$$

where $\int_{x_{R}}$ denotes the (clockwise) contour integral around $Q$.

(3.20) Definition. Given $P \in \mathscr{D}_{z}$, we define an operator $\Phi(P)$ acting on $\mathscr{F}$ and $\overline{\mathscr{F}}$ as follows:

$$
\Phi(P)=\frac{1}{2 \pi i} \int_{\infty_{2}} d z:(P \psi(z)) \bar{\psi}(z):=\frac{1}{2 \pi i} \int_{\infty} d z: \psi(z)\left(P^{\dagger} \bar{\psi}(z)\right): .
$$

This procedure is called the second quantization and this $\Phi(P)$ is called the secondquantized operator corresponding to $P$. The naming is justified from the next theorem.

(3.21) Theorem. 1) For $P \in \mathscr{D}_{z}$,

$$
\begin{aligned}
& {[\Phi(P), \psi(z)]=P \psi(z),} \\
& {[\Phi(P), \bar{\psi}(z)]=-P^{\dagger} \bar{\psi}(z) .}
\end{aligned}
$$

2) For $P, Q \in \Phi_{z}$,

where

$$
[\Phi(P), \Phi(Q)]=\Phi([Q, P])+c(P, Q) i d
$$

$$
c(P, Q)=\left(\frac{1}{2 \pi \sqrt{-1}}\right)^{2} \int_{\infty} d z \int_{\substack{\infty \\ z}} d w\left(P\left(w, \frac{d}{d w}\right) \frac{1}{w-z}\right)\left(Q\left(z, \frac{d}{d z}\right) \frac{1}{w-z}\right),
$$

called the Schwinger term. 
Proof. 1) From the convergence of the operator product we see that the contour should be chosen as follows;

$$
\begin{aligned}
& \Phi(P) \psi(z)=\frac{1}{2 \pi \sqrt{-1}} \int_{(x)} d w:(P \psi(w)) \bar{\psi}(w): \psi(z), \\
& \psi(z) \Phi(P)=\frac{1}{2 \pi \sqrt{-1}} \int_{x=2} d w \psi(z):(P \psi(w)) \bar{\psi}(w): .
\end{aligned}
$$

Then we can obtain the following relation

$$
\begin{aligned}
{[\Phi(P), \psi(z)] } & =\frac{1}{2 \pi \sqrt{-1}} \int_{\bar{z}} d w:(P \psi(w)) \bar{\psi}(w): \psi(z) \\
& =\frac{1}{2 \pi \sqrt{-1}} \int_{\bar{F}} d w(P \psi(w)) \bar{\psi}(w) \psi(z)=P \psi(z) .
\end{aligned}
$$

We can proceed in a similar way for $\bar{\psi}(z)$.

2) The commutator of $\Phi(P)$ and $\Phi(Q)$ can be expressed similarly as:

$$
[\Phi(P), \Phi(Q)]=\left(\frac{1}{2 \pi \sqrt{-1}}\right)^{2} \int_{\varnothing} d z \int_{(\mathcal{Q})} d w:(P \psi(w)) \bar{\psi}(w)::(Q \psi(z)) \bar{\psi}(z):
$$

Using Wick's theorem for the integrand and taking the singular parts into account, we obtain

$$
\begin{aligned}
& \text { (integrand) }=:\left(P_{w} \psi(w)\right) \bar{\psi}(w)::\left(Q_{z} \psi(z)\right) \bar{\psi}(z):+ \\
& \quad:\left(P_{w} \psi(w)\right) \bar{\psi}(w)::\left(Q_{z} \psi(z)\right) \bar{\psi}(z):+:\left(P_{w} \psi(w)\right) \bar{\psi}(w)::\left(Q_{z} \psi(z)\right) \bar{\psi}(z): .
\end{aligned}
$$

Then the first two terms correspond to $\Phi([Q, P])$ and the third term provides the Schwinger term $c(P, Q)$.

(3.22) Proposition [S.S]. The bilinear map $c: \mathscr{D}_{z} \times \mathscr{D}_{z} \rightarrow \mathbb{C}$ enjoys the following properties:

1) $c(P, Q)=-c(Q, P)$;

2) $c(P,[Q, R])+c(Q,[R, P])+c(R,[P, Q])=0$;

3) for the basis $u_{n}^{k} \equiv z^{k+n} \frac{1}{k !}\left(\frac{d}{d z}\right)^{k} \in \mathscr{D}_{z}, k \geqq 0, n \in \mathbb{Z}$,

$$
c\left(u_{n}^{k}, u_{m}^{\ell}\right)=(-1)^{k} \frac{1}{(k+\ell+1) !} \prod_{j=-\ell}^{k}(n+j) \delta_{m+n, 0} .
$$

From 1) and 2) the map $c ; \mathscr{D}_{z} \times \mathscr{D}_{z} \rightarrow \mathbb{C}$ determines a cohomology class $[c] \in H^{2}\left(\mathscr{D}_{z}, \mathbb{C}\right)$, which turns out to be nonzero cohomology class.

\section{D) Lifting of the Action of $\mathscr{D}_{z}$}

(3.23) Using $\Phi(P)$ defined in $C)$, we can lift the infinitesimal action $\theta(P)$ on UGM to $\tilde{\theta}(P)$ on $\widetilde{U G M}$, by defining it on $\mathscr{F}^{\times}$and by checking that it preserves the image of UGM by the Plücker embedding. 
(3.24) Definition. For each $P \in \mathscr{D}_{z}$ we define a vector field; $\widetilde{\theta}(P)$ on $\mathscr{F}^{\times}$in the following way:

where $|u\rangle \in \mathscr{F}^{\times}$.

$$
\tilde{\theta}_{|u\rangle}(P)=\Phi(P)|u\rangle \in T_{|u\rangle} \mathscr{F}^{\times} \stackrel{\sim}{\longrightarrow} \mathscr{F}
$$

(3.25) Let $\mathbb{I}$ be a one-dimensional subspace of $\mathscr{F}$ and $[\mathbb{L}]$ the corresponding point in $\mathbb{P}(\mathscr{F})$. Similarly as $(1.12)$ we have a canonical isomorphism

$$
T_{[\mathbb{L}]} \mathbb{P}(\mathscr{F}) \stackrel{\sim}{\longrightarrow} \operatorname{Hom}_{\mathbb{C}}(\mathbb{L}, \mathscr{F} / \mathbb{L}) .
$$

Then in the same way as (1.14) we define a holomorphic vector field $\theta(P) \in H^{0}(\mathbb{P}(\mathscr{F}), \Theta)$ for $P \in \mathscr{D}_{z}$ as

$$
\theta_{[\mathbb{L}]}(P): \mathbb{L} \subset \mathscr{F} \stackrel{\Phi(P)}{\longrightarrow} \mathscr{F} \longrightarrow \mathscr{F} / \mathbb{L} .
$$

For $\tilde{\theta}$ and $\theta$ we infer readily the following propositions.

(3.26) Definition. Let $F \rightarrow B$ be a $\mathbb{C}^{*}$-bundle. Then the action of $\mathbb{C}^{*}$ defines a holomorphic vector field $E$ on $F$ along the fibre, called the Euler operator. For $\mathscr{F}^{\times} \rightarrow \mathbb{P}(\mathscr{F}), E$ is nothing but the vector field corresponding to id: $\mathscr{F} \rightarrow \mathscr{F}$ with the above description.

(3.27) Proposition. Every $\widetilde{\theta}(P), P \in \mathscr{D}_{z}$, preserves the ideal $\mathscr{I}$ of $\mathcal{O}_{\mathscr{F}}$ generated by the Plücker relation:

$$
\tilde{\theta}(P) \mathscr{I} \subset \mathscr{I} .
$$

Therefore $\widetilde{\theta}(P)$ [respectively $\theta(P)$ ] defines a holomorphic vector field on $\widetilde{\mathrm{UGM}}$ (respectively UGM). This $\theta(P)$ coincides with the one defined in (1.14).

Summing up, we have obtained the following main theorem in this section.

(3.28) Theorem. For $P \in \mathscr{D}_{z}$ there are holomorphic vector fields $\theta(P), \tilde{\theta}(P)$ whose action is compatible with respect to the following diagram:

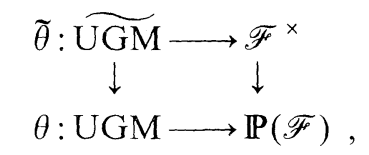

where $\theta$ on UGM is defined in (1.14).

(3.29) Corollary. For $P, Q \in \mathscr{D}_{z}$ we have

$$
[\tilde{\theta}(P), \tilde{\theta}(Q)]=\tilde{\theta}([Q, P])+c(P, Q) E
$$

\section{E) Current and Virasoro Algebra}

(3.30) We observe that $\widetilde{\mathfrak{G}}=\mathscr{D}^{1}(\hat{K}),(2.7)$, is a Lie subalgebra of $\mathscr{D}_{z}$. The operators $\Phi(P), P \in \widetilde{\mathfrak{b}}$, play the most essential role in our theory.

(3.31) Definition. 1) The following operators corresponding to scalar multiplications are called current operators:

$$
J_{n}=-\Phi\left(z^{n}\right)=-\frac{1}{2 \pi \sqrt{-1}} \int_{\text {ळ }} d t z^{n}: \psi(z) \bar{\psi}(z):
$$


Then we call

$$
J(z)=: \bar{\psi}(z) \psi(z):=\sum_{n \in \mathbb{Z}} J_{n} z^{-n-1}
$$

the current operator.

2) The following operators corresponding to derivations are called Virasoro operators $($ for $\operatorname{spin} j)$ :

$$
\begin{aligned}
L_{n}^{(j)} & =\Phi\left(s_{j}\left(z^{n+1} \frac{d}{d z}\right)\right)=\Phi\left(z^{n}\left(z \frac{d}{d z}+j(n+1)\right)\right) \\
& =\frac{1}{2 \pi \sqrt{-1}} \int_{\text {ळ }} d z z^{n+1}:\left\{(1-j) \frac{d \psi(z)}{d z} \bar{\psi}(z)-j \psi(z) \frac{d \bar{\psi}(z)}{d z}\right\}:
\end{aligned}
$$

for $j \in \frac{1}{2} \mathbb{Z}, n \in \mathbb{Z}$ [for $s_{j}$ see (2.32)]. Then we call

$$
T^{j}(z)=:(1-j) \frac{d \psi(z)}{d z} \bar{\psi}(z)-j \psi(z) \frac{d \bar{\psi}(z)}{d z}:=\sum_{n \in \mathbb{Z}} L_{n}^{(j)} z^{-n-2}
$$

the energy momentum tensor (for spin $j$-fields).

3) The Lie subalgebra generated by id and $\left\{J_{n}\right\}$ (respectively $L_{n}^{(j)}$ ) is called the current algebra (respectively the Virasoro algebra). For $j=1 / 2$ we omit the superindex $j$ in the notation of $L$ and $T$ hereafter.

(3.32). Formulae. From (3.21) we obtain the following fundamental relations of these operators:

$$
\begin{aligned}
& {\left[J_{n}, J_{m}\right]=n \delta_{n+m, 0},} \\
& {\left[L_{n}^{(j)}, L_{m}^{(j)}\right]=(n-m) L_{n+m}^{(j)}-\frac{1}{6}\left(6 j^{2}-6 j+1\right)\left(n^{3}-n\right) \delta_{n+m, 0},} \\
& {\left[L_{n}^{(j)}, J_{m}\right]=-m J_{n+m}-\frac{1}{2}(2 j-1)\left(n^{2}+n\right) \delta_{n+m, 0},} \\
& {\left[J_{n}, \psi(z)\right]=-z^{n} \psi(z)} \\
& {\left[J_{n}, \bar{\psi}(z)\right]=z^{n} \bar{\psi}(z)} \\
& {\left[L_{n}^{(j)}, \psi(z)\right]=z^{n}\left(z \frac{d}{d z}+j(n+1)\right) \psi(z),} \\
& {\left[L_{n}^{(j)}, \bar{\psi}(z)\right]=z^{n}\left(z \frac{d}{d z}+(1-j)(n+1)\right) \bar{\psi}(z) .}
\end{aligned}
$$

Moreover between $T(z)$ and $J(z)$ there is an important relation.

(3.33) Proposition (Sugawara form of energy-momentum tensor).

$$
T(z)=\frac{1}{2}: J(z) J(z)^{\circ}=\frac{1}{2} \sum_{m, n \in \mathbb{Z}} \circ J_{m} J_{n-m^{\circ} z^{-n-2},}
$$

where: $:$ is the ordering which transforms $J_{n}(n \geqq 0)$ to the right and $J_{n}(n<0)$ to the left (see Sect. 4,A)). 
(3.34) Proposition. There exist unique maps:

$$
\tilde{\theta}: \mathfrak{G} \rightarrow H^{0}\left(\tilde{\mathscr{C}}_{g}(\tilde{\mathscr{F}}), \Theta\right) \quad \text { and } \quad \tilde{\theta}: \widetilde{\mathfrak{F}} \rightarrow H^{0}\left(\widetilde{\mathbb{P}}\left(\hat{\mathscr{X}}_{g}^{(0)}\right), \Theta\right)
$$

(holomorphic vector fields) such that

1) $[\widetilde{\theta}(\ell), E]=0, \forall \ell \in \mathfrak{F}$ or $\widetilde{\mathfrak{G}}$,

2) Their actions are compatible, as indicated in the following diagram:

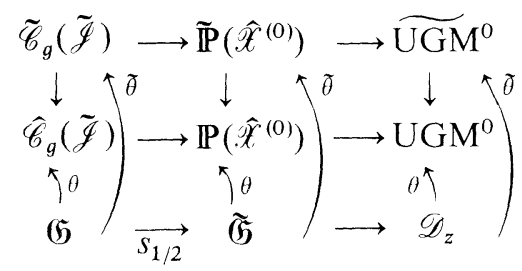

(3.35) Proposition. By the above compatibility and definition (3.24), it follows that

$$
\tilde{\theta}(\ell)\left|\tilde{X}_{c}\right\rangle=\Phi\left(s_{1 / 2} \circ \ell\right)\left|\tilde{X}_{c}\right\rangle
$$

for $\forall \tilde{X}_{c} \in \tilde{\mathscr{C}}_{g}(\tilde{\mathscr{J}})$ and $\forall \ell \in \mathfrak{G}$.

This equation plays an essential role in Sect. 7.

\section{4. $\tau$-Function and Wave Function}

(4.0) In this section we reformulate the Sato theory of KP equations into a convenient form for our later use. We give sketchy but selfcontained proof here. For more details about KP equations and related topics, see for example [Sa.; D.J.K.M.].

\section{A) Bosonization}

(4.1) We first explain one of the most fundamental principles of two-dimensional field theory: fermion-boson correspondence. The fermion Fock space $\mathscr{F}$ is isomorphic to a corresponding boson Fock space $\mathscr{H}$ defined below and the operators acting on these spaces have also a well-defined correspondence.

The boson Fock space is defined as the following vector space:

$$
\begin{aligned}
\mathscr{H}=\mathbb{C}\left[\left[t_{1}, t_{2}, \ldots\right]\right] \otimes \mathbb{C}\left[e^{t_{0}}, e^{-t_{0}}\right] & =\sum_{m \in \mathbb{Z}} \mathbb{C}\left[\left[t_{1}, t_{2}, \ldots\right]\right] e^{m t_{0}} \\
& =\sum_{m \in \mathbb{Z}} \mathscr{H}_{m},
\end{aligned}
$$

where $t_{i}$ 's are infinite number of indeterminates. An element of $\mathscr{H}$ is called a boson state vector. We define the degree and the charge as follows:

$$
\begin{gathered}
\operatorname{deg} t_{i}=i, \quad \operatorname{deg} e^{t_{0}}=0, \\
\text { charge } t_{i}=0(i \neq 0), \quad \operatorname{charge} e^{n t_{0}}=n,
\end{gathered}
$$


then $\mathscr{H}$ decomposes into the charged sector $\mathscr{H}_{n}(n \in \mathbb{Z})$, each of which is a complete Hausdorff vector space by the filtration: $F^{d} \mathscr{H}_{n}=\prod_{d^{\prime} \geqq d} \mathscr{H}_{n}\left(d^{\prime}\right), \mathscr{H}_{n}(d)=\left\{f(\mathbb{T}) \in \mathscr{H}_{n} ;\right.$
degree $f(\mathbb{T})=d\}$.

(4.2) Definition. We define a linear map $B: \mathscr{F} \rightarrow \mathscr{H}$ as follows:

$$
B|\Psi\rangle=\sum_{n \in \mathbb{Z}} e^{n t_{0}}\left\langle n\left|\exp \left(\sum_{m=1}^{\infty} J_{m} t_{m}\right)\right| \Psi\right\rangle
$$

(4.3) Theorem (Bosonization [D.J.K.M.]. 1) The linear map $B: \mathscr{F} \rightarrow \mathscr{H}$ gives a topological isomorphism.

$$
B: \mathscr{\mathscr { F }} \stackrel{\mathscr{H}}{\simeq} .
$$

2) $B$ preserves the charge and the degree

$$
\mathscr{F}_{n}(d) \stackrel{\simeq}{\longrightarrow} \mathscr{H}_{n}(d)
$$

(4.4) We define the following fundamental operators on $\mathscr{H}$ :

$$
a_{n}=\frac{\partial}{\partial t_{n}}(n \geqq 0), \quad a_{-n}=n t_{n}(n>0), \quad q=t_{0} .
$$

The following relations can be easily confirmed:

$$
\left[a_{m}, a_{n}\right]=m \delta_{m+n, 0}, \quad\left[a_{m}, e^{q}\right]=e^{q} \delta_{m, 0},
$$

which are standard bosonic commutation relations.

The normal ordering $: \circ$ of these operators, $a_{m}$ and $e^{n q}$, are defined as follows:

1) Inside the symbol : : we put polynomials of $a_{n}(n \in \mathbb{Z})$ and $e^{q}$, and all the operators $a_{n}$ and $e^{q}$ commute each other.

2) In case the operators inside the symbol $::$ are in normal order [i.e. all the creation operators $\left(a_{n}(n<0), e^{q}\right)$ are located to the left of all the annihilation operators $\left.\left(a_{n}(n \geqq 0)\right)\right]$. The symbol can be taken away and the remaining operators are considered as those acting on $\mathscr{H}$. Fro example $: a_{n} a_{-m} \circ{ }^{\circ} a_{-m} a_{n}^{\circ}=a_{-m} a_{n}$, for $n, m>0$.

Each operator $\mathcal{O}$ on the fermion Fock space has the corresponding one $\mathcal{O}_{B}=B \mathcal{O} B^{-1}$ on the boson Fock space.

We introduce the following field operator which is familiar in string theory:

$$
Q(z)=q+a_{0} \log z-\sum_{n \neq 0} \frac{a_{n}}{n} z^{-n}
$$

and set for $k \in \mathbb{Z}$

$$
V_{k}(z)=\circ e^{k Q(z) \circ}=\exp \left(k \sum_{n=1}^{\infty} t_{n} z^{n}\right) e^{k t_{0}} \exp \left(k \log z \frac{\partial}{\partial t_{0}}-k \sum_{n=1}^{\infty} \frac{z^{-n}}{n} \frac{\partial}{\partial t_{n}}\right),
$$

which we call a vertex operator of charge $k$. 
Then we have the following:

(4.5) Theorem (Fermion-boson correspondence).

$$
\begin{aligned}
& \psi_{B}(z)=V_{-1}(z), \\
& \bar{\psi}_{B}(z)=V_{+1}(z), \\
& J_{B}(z)=A(z)=\frac{d Q(z)}{d z}=\sum_{n \in \mathbb{Z}} a_{n} z^{-n-1}, \\
& T_{B}(z)=\frac{1}{2} \circ A(z) A(z) \circ,
\end{aligned}
$$

where $J_{B}(z)$ and $T_{B}(z)$ are the bosonized version of the current operator and the energymomentum tensor operator.

\section{B) $\tau$-Functions and Wave Functions}

(4.6) We first take an element $\tilde{U}\left(\in{\widetilde{\mathrm{UGM}^{\phi}}}^{\phi}\right)$ [cf. (1.20)] of the charge zero sector of the universal Grassmann manifold and its image in the Fock space $|\widetilde{U}\rangle \in \mathscr{F}_{0}{ }^{\times}$. We then define the following quantity:

(4.7) Definitions.

$$
\begin{aligned}
\tau(\mathbb{T}, \tilde{U}) & =\left\langle 0\left|e^{H(\mathbb{T})}\right| \tilde{U}\right\rangle, \\
\Psi(z, \mathbb{T}, U) & =\frac{\left\langle-1\left|e^{H(\mathbb{T})} \psi(z)\right| \tilde{U}\right\rangle}{\tau(\mathbb{T}, \tilde{U})} \\
\bar{\Psi}(z, \mathbb{T}, U) & =\frac{\left\langle 1\left|e^{H(\mathbb{T})} \bar{\psi}(z)\right| \tilde{U}\right\rangle}{\tau(\mathbb{T}, \tilde{U})},
\end{aligned}
$$

where

$$
H(\mathbb{T})=\sum_{n=1}^{\infty} t_{n} J_{n}
$$

We call $\tau(\mathbb{T}, \tilde{U})$ a $\tau$-function and $\Psi(z, \mathbb{T}, U), \widetilde{\Psi}(z, \mathbb{T}, U)$ (conjugate-) wave functions associated with $\tilde{U} \in \widetilde{U G M}^{\phi}$. Note that $\Psi, \widetilde{\Psi}$ depend only on $U=\pi(\tilde{U}) \in \mathrm{UGM}^{\phi}$. Remark. We can define $\tau(\mathbb{T}, \tilde{U})$ for $\tilde{U} \in \widetilde{U G M}^{0}$ as above. Then $\tau(0, \tilde{U}) \neq 0$ if and only if $\tilde{U} \in \widetilde{U G M}^{\phi}$.

We provide several important properties of the (conjugate-)wave functions.

(4.8) Proposition [D.J.K.M.]. 1) The wave functions have the following expansions:

$$
\begin{aligned}
& \Psi(z, \mathbb{T}, U)=e^{-\xi(\mathbb{T}, z)}\left(1+\sum_{k=1}^{\infty} w_{k}(\mathbb{T}) z^{-k}\right), \\
& \Psi(z, \mathbb{T}, U)=e^{\xi(\mathbb{T}, z)}\left(1+\sum_{k=1}^{\infty} \bar{w}_{k}(\mathbb{T}) z^{-k}\right),
\end{aligned}
$$

where $w_{k}(t), \bar{w}_{k}(t) \in \mathbb{C}[[\mathbb{T}]]$ and $\xi(\mathbb{T}, z)=\sum_{n=1}^{\infty} t_{n} z^{n}$. 
2) If we write

$$
\begin{aligned}
& \Psi(z, \mathbb{T}, U)=\sum_{M} \Psi_{M}(z, U) \mathbb{T}^{M} \\
& \text { (respectively } \bar{\Psi}(z, \mathbb{T}, U)=\sum_{M} \bar{\Psi}_{M}(z, U) \mathbb{T}^{M} \text { ), }
\end{aligned}
$$

then we have $\Psi_{M}(z, U) \in U$ (respectively $\left.\bar{\Psi}_{M}(z, U) \in \bar{U}\right)$, where

$$
\mathbb{T}^{M}=t_{1}^{m_{1}} t_{2}^{m_{2}} t_{3}^{m_{3}} \ldots, \quad M=\left(m_{1}, m_{2}, m_{3}, \ldots\right), \quad m_{i}(\geqq 0) \in \mathbb{Z} .
$$

Proof. 1) The expansion 1) can be obtained easily with the bosonization rule mentioned in (4.5);

$$
\begin{aligned}
& \Psi(z, \mathbb{T}, U)=e^{-\xi(\mathbb{T}, z)} \frac{\tau(\mathbb{T}+[z], \tilde{U})}{\tau(\mathbb{T}, \widetilde{U})}, \\
& \bar{\Psi}(z, \mathbb{T}, U)=e^{\xi(\mathbb{T}, z)} \frac{\tau(\mathbb{T}-[z], \tilde{U})}{\tau(\mathbb{T}, \tilde{U})},
\end{aligned}
$$

where $[z]=\left(\frac{1}{z}, \frac{1}{2 z^{2}}, \frac{1}{3 z^{3}}, \ldots\right)$.

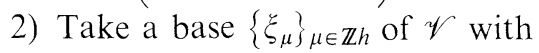

$$
\xi^{\mu} \in U(\mu<0), \quad \xi^{\mu}=e^{\mu}(\mu>0)
$$

and satisfying the following relation:

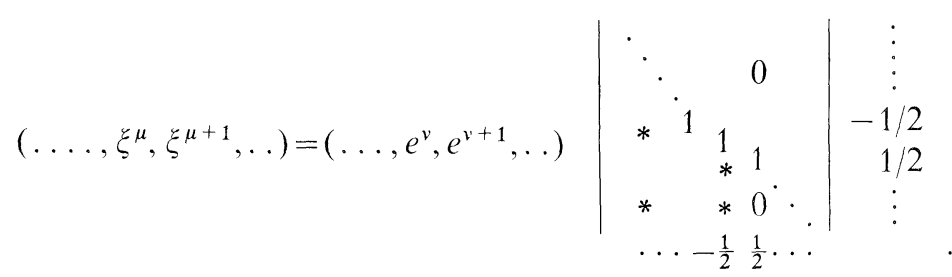

Identifying the matrix in the right-hand side of the above equality with an element $g$ of $P_{-}$and the corresponding operator on the Fock space [see (1.26)] as $G[g$ ] (i.e. $|\tilde{U}\rangle=G[g]|0\rangle$ ) we obtain the following relation:

$$
G^{-1}\left(\sum_{\mu \in \mathbb{Z} h} \psi_{\mu} e^{\mu}\right) G=\sum_{\mu \in \mathbb{Z} h}\left(G^{-1} \psi_{\mu} G\right) e^{\mu}=\sum_{\mu, v \in \mathbb{Z} h} \psi_{\nu} g_{\mu}^{v} e^{\mu}=\sum_{\nu \in \mathbb{Z} h} \psi_{\nu} \xi^{v}
$$

We then obtain

$$
\psi(z)|\tilde{U}\rangle=\left(\sum_{\mu \in \mathbb{Z} h} \psi_{\mu} e^{\mu}\right) G|0\rangle=G\left(\sum_{\mu \in \mathbb{Z} h} \psi_{\mu} \xi^{\mu}\right)|0\rangle=\sum_{\mu<0}\left(G \psi_{\mu}|0\rangle\right) \xi^{\mu}
$$

Bosonizing these relations, we obtain the result:

$$
\Psi_{M}(z, U) \in U \text {. }
$$

The argument is the same for the conjugate sector. 
Since $U$ and $\bar{U}\left(=U^{\perp}\right)$ are orthogonal to each other with respect to $\operatorname{Res}_{z=\infty}$, the following relations are a natural consequence of 2$)$ :

(4.9) Corollary (Bilinear Relation).

$$
\operatorname{Res}_{z=\infty} \Psi(z, \mathbb{T}, U) \bar{\Psi}\left(z, \mathbb{T}^{\prime}, U\right)=0 .
$$

(4.10) Corollary (Hirota's Bilinear Equation for $\tau$-function) [H.].

$$
\sum_{j=0}^{\infty} P_{j}(-2 \mathbb{Y}) P_{j+1}\left(\tilde{D}_{\mathbb{T}}\right) \exp \left(\sum_{\ell=1}^{\infty} y_{\ell} D_{t_{\ell}}\right) \tau(\mathbb{T}) \cdot \tau(\mathbb{T})=0,
$$

where

$$
\exp \left(\sum_{n=1}^{\infty} t_{n} z^{n}\right)=\sum_{j=0}^{\infty} P_{j}(\mathbb{T}) z^{j}, \quad \tilde{D}_{\mathbb{T}}=\left(D_{t_{1}}, \frac{1}{2} D_{t_{2}}, \frac{1}{3} D_{t_{3}}, \ldots\right)
$$

and

$$
P\left(D_{\mathbb{T}}\right) f(\mathbb{T}) \cdot g(\mathbb{T})=\left[P\left(\partial_{\mathbb{Y}}\right)\{f(\mathbb{T}+\mathbb{Y}) g(\mathbb{T}-\mathbb{Y})\}\right]_{\mathbb{Y}=0} .
$$

This equation is obtained as a direct consequence of the bilinear relation. It characterizes that the $\tau$-function is associated with an element of UGM (Theorem 4.16). (See [D.J.K.M.] for more details about (4.9) and (4.10).)

(4.11) Remark. The bilinear relation (4.9) is nothing but the bosonized version of the Plücker relation, and its fermionic form is represented as

$$
\operatorname{Res}_{z=\infty} \psi(z)|U\rangle \otimes \bar{\psi}(z)|U\rangle=\sum_{\mu \in \mathbb{Z} h} \psi_{\mu}|U\rangle \otimes \bar{\psi}_{-\mu}|U\rangle=0
$$

C) $\tau$-Function

(4.12) Putting aside the definitions of $\tau, \Psi$ and $\bar{\Psi}$ given in B) for a while, we investigate how the properties given in Proposition (4.8) of B) characterize $\Psi$ and $\bar{\Psi}$.

(4.13) Definition. (Generic Wave Function). We call

$$
\Psi(z, \mathbb{T})=\sum_{N} \Psi_{N}(z) \mathbb{T}^{N}, \bar{\Psi}(z, \mathbb{T})=\sum_{N} \bar{\Psi}_{N}(z) \mathbb{T}^{N} \in \mathbb{C}\left(\left(z^{-1}\right)\right) \hat{\otimes} \mathbb{C}[[\mathbb{T}]]
$$

a conjugate pair of generic wave functions when they satisfy the following two conditions:

1) asymptotic behavior

$$
\begin{array}{ll}
\Psi(z, \mathbb{T})=e^{-\xi(z, \mathbb{T})}\left(1+\sum_{k=1}^{\infty} w_{k}(\mathbb{T}) z^{-k}\right), & w_{k}(\mathbb{T}) \in \mathbb{C}[[\mathbb{T}]], \\
\bar{\Psi}(z, \mathbb{T})=e^{\xi(z, \mathbb{T})}\left(1+\sum_{k=1}^{\infty} \bar{w}_{k}(\mathbb{T}) z^{-k}\right), & \bar{w}_{k}(\mathbb{T}) \in \mathbb{C}[[\mathbb{T}]]
\end{array}
$$

2) bilinear relation

$$
\operatorname{Res}_{z=\infty} \Psi(z, \mathbb{T}) \widetilde{\Psi}\left(z, \mathbb{T}^{\prime}\right)=\sum_{M, N} \mathbb{T}^{M} \mathbb{T}^{\prime N} \operatorname{Res}_{z=\infty} \Psi_{M}(z) \bar{\Psi}_{N}(z)=0
$$

We denote the whole set of pairs $\Psi, \bar{\Psi}$ satisfying 1) and 2) by $W F^{\phi}$. 
(4.14) Definition (Generic $\tau$-function). $\tau(\mathbb{T}) \in \mathbb{C}[[\mathbb{T}]]$ is called the generic $\tau$-function when it satisfies the following conditions:

1) $\tau(\mathbb{T})$ satisfies the Hirota's bilinear equation [Corollary (4.10)],

2) $\tau(\mathbb{D}) \neq 0$.

We denote the whole set of generic $\tau$-functions by $T F^{\phi}$.

(4.15) Proposition. 1) For any pair $(\Psi(z, \mathbb{T}), \bar{\Psi}(z, \mathbb{T})) \in W F^{\phi}$, there exists a unique element $U \in \mathrm{UGM}^{\phi}$ such that $\Psi_{N}(z) \in U, \bar{\Psi}_{N}(z) \in \bar{U}\left(=U^{\perp}\right)$.

2) The map $W F^{\phi} \rightarrow \mathrm{UGM}^{\phi}$ given above is bijective.

Proof. 1) The terms $w_{n}(\mathbb{T})$ and $\bar{w}_{n}(\mathbb{T})$ in the asymptotic expansion of $\Psi(z, \mathbb{T}), \bar{\Psi}(z, \mathbb{T})$ can be expanded with respect to $t_{j}$ as follows:

$$
\begin{aligned}
& w_{n}(\mathbb{T})=c_{n}+\sum_{j=1}^{\infty} c_{n j} t_{j}+o\left(\mathbb{T}^{2}\right), \\
& \bar{w}_{n}(\mathbb{T})=\bar{c}_{n}+\sum_{j=1}^{\infty} \bar{c}_{n j} t_{j}+o\left(\mathbb{T}^{2}\right) .
\end{aligned}
$$

Define elements in $\mathbb{C}\left(\left(z^{-1}\right)\right)$ as follows :

$$
\begin{aligned}
& \varphi_{0}(z) \equiv \Psi_{N_{0}}(z)=1+\sum_{n=1}^{\infty} c_{n} z^{-n}, \\
& \varphi_{j}(z) \equiv \Psi_{N_{j}}(z)=-z^{j}\left(1+\sum_{n=1}^{\infty} c_{n} z^{-n}\right)+\sum_{n=1}^{\infty} c_{n j} z^{-n} \quad(j \geqq 1), \\
& \bar{\varphi}_{0}(z) \equiv \bar{\Psi}_{N_{0}}(z)=1+\sum_{n=1}^{\infty} \bar{c}_{n} z^{-n}, \\
& \bar{\varphi}_{j}(z) \equiv \bar{\Psi}_{N_{j}}(z)=z^{j}\left(1+\sum_{n=1}^{\infty} \bar{c}_{n} z^{-n}\right)+\sum_{n=1}^{\infty} \bar{c}_{n j} z^{-n} \quad(j \geqq 1),
\end{aligned}
$$

where

$$
\begin{aligned}
& N_{0}=(0,0,0, \ldots), \\
& N_{j}=(0,0, \ldots, 0, \stackrel{v}{1}, 0, \ldots),
\end{aligned}
$$

and define the following vector subspaces:

$$
\begin{aligned}
& U=\sum_{n=0}^{\infty} \mathbb{C} \varphi_{n}(z) \subseteq \hat{K}, \\
& \bar{U}=\sum_{n=0}^{\infty} \mathbb{C} \bar{\varphi}_{n}(z) \subseteq \hat{K} .
\end{aligned}
$$

As a direct consequence of the bilinear relation (4.10) we obtain (1) $\bar{U} \subseteq U^{\perp}, U \subseteq \bar{U}^{\perp}$. The concrete expression $\varphi_{n}(z)$ and $\bar{\varphi}_{n}(z)$ given above naturally leads to (2) $U^{\perp} \cap F^{0} \overline{\mathscr{V}}=0, \bar{U}^{\perp} \cap F^{0} \mathscr{V}=0$. Recalling $M(U)=M(\bar{U})=\left\{-\frac{1}{2},-\frac{3}{2}, \ldots\right\}$ [cf. (1.7), (1.11)], we can show that (3) $\mathscr{V}=U \oplus F^{0} \mathscr{V}, \overline{\mathscr{V}}=\bar{U} \oplus F^{0} \overline{\mathscr{V}}$. (1), (2) and (3) imply 
$\bar{U}=U^{\perp}, U=\bar{U}^{\perp}$. Then by the bilinear relation we obtain the desired result:

$$
\Psi_{N}(z) \in \bar{U}^{\perp}=U, \quad \bar{\Psi}_{N}(z) \in U^{\perp}=\bar{U} \quad \text { for } \forall N .
$$

It is obvious from (2) that $U \in \mathrm{UGM}^{\phi}$.

2) We now show that two pairs of wave functions $(\Psi(z, \mathbb{T}), \bar{\Psi}(z, \mathbb{T}))$, $\left(\Psi^{\prime}(z, \mathbb{T}), \bar{\Psi}^{\prime}(z, \mathbb{T})\right) \in W F^{\phi}$ corresponding to the same $U \in \mathrm{UGM}^{\phi}$ coincide with each other. For this purpose we expand the wave functions as follows:

$$
\Psi(z, \mathbb{T})=e^{-\xi(z, \mathbb{T})} \sum_{n=0}^{\infty} \sum_{|N|=n} w_{n}(z) \mathbb{T}^{N},
$$

where

i.e.

$$
w_{N}(z) \in \mathbb{C}\left[\left[z^{-1}\right]\right] z^{-1}=F^{0} \mathscr{V} \text { for }|N|>0
$$

$$
\Psi_{N}(z)=\left[e^{-\xi(z, \mathbb{T})} \sum_{|M|<|N|} w_{N}(z) \mathbb{T}^{M}\right]_{N}+w_{N}(z)
$$

with $|N|=\sum_{i=1}^{\infty} n_{i}$. By induction with respect to $|N|$ we show $w_{N}(z)=w_{N}^{\prime}(z)$. The argument works similarly for the conjugate sector.

i) For $|N|=0$ (i.e. $N=N_{0}$ ) we obtain

$$
\begin{aligned}
& w_{N_{0}}(z)=1+\sum_{n=1}^{\infty} c_{n} z^{-n}=\varphi_{0}(z) \in F^{-1} U, \\
& w_{N_{0}}^{\prime}(z)=1+\sum_{n=1}^{\infty} c_{n}^{\prime} z^{-n}=\varphi_{0}^{\prime}(z) \in F^{-1} U .
\end{aligned}
$$

Since $\operatorname{dim} F^{-1} U=1$, we obtain

$$
w_{N_{0}}(z)=w_{N_{0}}^{\prime}(z) \text {. }
$$

ii) Assuming $w_{M}(z)=w_{M}^{\prime}(z)$ for $|M|<n$, we obtain

$$
\varphi \equiv \Psi_{N}(z)-\Psi_{N}^{\prime}(z)=w_{N}(z)-w_{N}^{\prime}(z) \in F^{0} \mathscr{V} \cap U=\{0\},
$$

for $|N|=n$.

Conversely the mapping $\mathrm{UGM}^{\phi} \rightarrow W F^{\phi}$ is shown to be surjective because of the correspondence of equations in (4.7) and Proposition (4.8).

Define the map $T F^{\phi} / \mathbb{C}^{*} \rightarrow W F^{\phi}$ by

$$
\begin{aligned}
& \Psi(\mathbb{T}, z)=e^{-\xi(z, \mathbb{T}) \frac{\tau(\mathbb{T}+[z])}{\tau(\mathbb{T})},} \\
& \bar{\Psi}(\mathbb{T}, z)=e^{\xi(z, \mathbb{T})} \frac{\tau(\mathbb{T}-[z])}{\tau(\mathbb{T})} .
\end{aligned}
$$

(4.16) Theorem. The following diagram is commutative and all arrows are bijections:

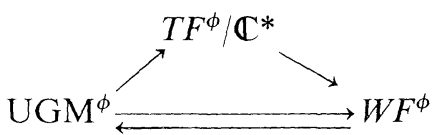


Proof. Only injectivity of the mapping $T F^{\phi} / \mathbb{C}^{*} \rightarrow W F^{\phi}$ is nontrivial. From Proposition (4.15) $\Psi(z, \mathbb{T})$ and $\bar{\Psi}(z, \mathbb{T})$ are uniquely characterized by the condition $\Psi_{N}(z) \in U, \bar{\Psi}_{N}(z) \in \bar{U}$ for $\forall N$. If $\Psi(z, \mathbb{T})$ and $\bar{\Psi}(z, \mathbb{T})$ are expressed by a $\tau$-function $\tau(\mathbb{T})$ as $(* *)$, then the above conditions for $\Psi(z, \mathbb{T})$ and $\bar{\Psi}(z, \mathbb{T})$ are equivalent to the following conditions on $\tau(\mathbb{T})$ :

$$
\begin{array}{lll}
\operatorname{Res}_{z=\infty}\left(\bar{\xi}(z) \psi_{B}(z)\right) \tau(\mathbb{T})=0 & \text { for } \quad \forall \bar{\xi}(z) \in \bar{U}, \\
\operatorname{Res}_{z=\infty}\left(\xi(\mathrm{z}) \bar{\psi}_{B}(\mathrm{z})\right) \tau(\mathbb{T})=0 & \text { for } \quad \forall \xi(z) \in U .
\end{array}
$$

These conditions determine $\tau(\mathbb{T})$ uniquely up to a constant by using the characterization of $|\tilde{U}\rangle$ by $\mathscr{W}_{+}(U) \oplus \overline{\mathscr{W}}_{+}(U)$ established in Theorem (3.13). Thus the mapping $T F^{\phi} / \mathbb{C}^{*} \rightarrow W F^{\phi}$ is injective.

Remark that the $\tau$-function $\tau(\mathbb{T})$ corresponding to $U \in \mathrm{UGM}^{\phi}$ is characterized by the following infinite order differential equations:

$$
\begin{aligned}
& \operatorname{Res}_{z=\infty}\left(\bar{\xi}(z) V_{-1}(\mathbb{T})\right) \tau(\mathbb{T})=0, \\
& \operatorname{Res}_{z=\infty}\left(\xi(z) V_{1}(\mathbb{T})\right) \tau(\mathbb{T})=0 .
\end{aligned}
$$

For simplicity we have restricted the discussions in the generic case $\left(\mathrm{UGM}^{\phi}\right)$. We can generalize the discussions to the whole $\mathrm{UGM}^{\phi}$.

(4.17) Theorem. The following two conditions are equivalent:

1) $f(\mathbb{T}) \in \mathscr{H}_{0}^{\times}$satisfies Hirota's bilinear equation (4.10)

$$
\sum_{j=0}^{\infty} P_{j}(-2 \mathbb{Y}) P_{j+1}\left(\tilde{D}_{\mathbb{T}}\right) \exp \left(\sum_{\ell=1}^{\infty} y_{\ell} D_{t_{\ell}}\right) \tau(\mathbb{T}) \cdot \tau(\mathbb{T})=0 ;
$$

2) there exists an element $\tilde{U} \in \widetilde{\mathrm{UGM}^{0}}$ such that $f(\mathbb{T})=\tau(\mathbb{T}, \tilde{U})$.

\section{The $\tau$-Function as a Period Map}

(5.0) In the preceding sections we have constructed a sequence of $\mathbb{C}^{*}$-bundles in the following (cartesian) diagram:

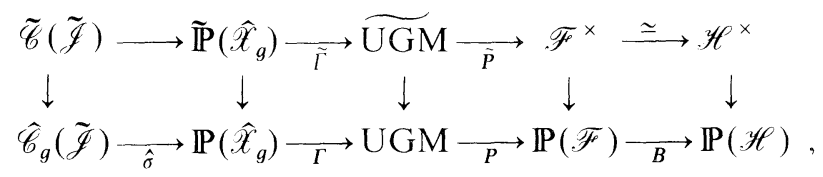

$\tilde{\mathscr{C}}_{g}(\tilde{\mathscr{J}})$ is the pull-back bundle.

We now define a holomorphic mapping $\Delta_{1 / 2}: \hat{\mathscr{C}}_{g}(\tilde{\mathscr{F}}) \rightarrow \mathbb{P}\left(\mathscr{H}_{0}\right)$ by

$$
\Delta_{1 / 2}: \hat{\mathscr{C}}_{g}(\tilde{\mathscr{J}}) \underset{\Gamma \circ \vec{\sigma}}{\longrightarrow} \mathrm{UGM}^{0} \underset{P}{\longrightarrow} \mathbb{P}\left(\mathscr{\mathscr { F }}_{0}\right) \longrightarrow \mathbb{P}\left(\mathscr{H}_{0}\right) .
$$

(5.1) The Fundamental Problem. Construct a section of $\hat{\mathscr{C}}_{g}(\tilde{\mathscr{J}})$, equivalently, a nonzero holomorphic function $\tau: \hat{\mathscr{C}}_{g}(\tilde{\mathscr{F}}) \rightarrow \mathscr{H}_{0}^{\times}$which makes the following diagram 
commutative:

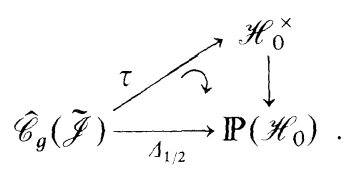

We actually construct a lifting $\tau$ by using the theory of KP equations. Geometrically $\tau$ is a period map of the moduli space $\hat{\mathscr{C}}_{g}(\tilde{\mathscr{J}})$. For a given lifting $\tau=\tau\left(\mathbb{T}, X_{c}\right)$ we can construct another lifting of $\Delta_{1 / 2}$ by $\tau^{\prime}(\mathbb{T}, X)=C\left(X_{c}\right) \tau(\mathbb{T}, X)$ for any holomorphic function $C: \hat{\mathscr{C}}_{g}(\tilde{\mathscr{J}}) \rightarrow \mathbb{C}^{*}$. In this sense the lifting $\tau$ is not unique (see Sect. 7,D)).

\section{A) Baker-Akhiezer Function}

(5.2) In this paragraph we reformulate the theory of the Baker-Akhiezer function (BA-function) of KP equations. For the original theory of the BA-function see [Kr.; D.].

(5.3) Definition. For a given data $X_{c}=\left(R,(\alpha, \beta), Q, u, \mathscr{L}_{c}\right) \in \hat{\mathscr{C}}_{g}(\tilde{\mathscr{F}})$, we define $\left.\Lambda\left(X_{c}\right), \bar{\Lambda}\left(X_{c}\right) \in \mathbb{C}\left(\left(z^{-1}\right)\right) \hat{\otimes} \mathbb{C}[[T]]\right]$ as follows :

$$
\begin{gathered}
\Lambda\left(X_{c}\right) \equiv\left\{\Psi(z, \mathbb{T})=\sum_{N} \Psi_{N}(z) \mathbb{T}^{N} \mid\right. \\
\Psi_{N}(z) \in U\left(X_{c}\right)=\sqrt{d u} H^{0}\left(R, \mathscr{L}_{\Delta} \otimes \mathscr{L}_{c}(* Q)\right), \\
\left.\Psi(z, \mathbb{T})=e^{-\xi(z, \mathbb{T})}\left(\sum_{k=0}^{\infty} w_{k}(\mathbb{T}) z^{-k}\right), \quad w_{k}(\mathbb{T}) \in \mathbb{C}[[\mathbb{T}]]\right\}, \\
\bar{\Lambda}\left(X_{c}\right) \equiv\left\{\bar{\Psi}(z, \mathbb{T})=\sum_{N} \bar{\Psi}_{N}(z) \mathbb{T}^{N} \mid\right. \\
\bar{\Psi}_{N}(z) \in \bar{U}\left(X_{c}\right)=\sqrt{d u} H^{0}\left(R, \mathscr{L}_{\Delta} \otimes \mathscr{L}_{c}^{-1}(* Q)\right), \\
\left.\bar{\Psi}(z, \mathbb{T})=e^{\xi(z, \mathbb{T})}\left(\sum_{k=0}^{\infty} \bar{w}_{k}(\mathbb{T}) z^{-h}\right), \quad \bar{w}_{k}(\mathbb{T}) \in \mathbb{C}[[\mathbb{T}]]\right\} .
\end{gathered}
$$

We call an element of $\Lambda\left(X_{c}\right)\left(\bar{\Lambda}\left(X_{c}\right)\right)$ as a (conjugate) Baker-Akhiezer function associated with the data $X_{c}$. We can then derive the following theorem. We essentially follow the proof of Krichever and Dubrovin.

(5.4) Theorem $\left[\mathrm{Kr} . ;\right.$ D.]. For a generic data $X_{c}=\left(R,(\alpha, \beta), Q, u, \mathscr{L}_{c}\right)$, i.e. $\Theta(c \mid \Omega) \neq 0$,

1) $\Lambda\left(X_{c}\right)$ is a free $\mathbb{C}[[\mathbb{T}]]$-module of rank 1 and generated by

$$
\Phi\left(z, \mathbb{T}, X_{c}\right)=f(z) \exp \left(-\sum_{n=1}^{\infty} t_{n} \varphi^{(n)}(z)\right) \frac{\Theta(I(\mathbb{T})+I[z]+c \mid \Omega)}{\Theta(I(\mathbb{T})+c \mid \Omega)},
$$

2) $\bar{\Lambda}\left(X_{c}\right)$ is a free $\mathbb{C}[[\mathbb{T}]]-m o d u l e$ of rank 1 and generated by

$$
\bar{\Phi}\left(z, \mathbb{T}, X_{c}\right)=f(z) \exp \left(\sum_{n=1}^{\infty} t_{n} \varphi^{(n)}(z)\right) \frac{\Theta(I(\mathbb{T})-I[z]+c \mid \Omega)}{\Theta(I(\mathbb{T})+c \mid \Omega)},
$$


where $I(\mathbb{T})=\left(I^{1}(\mathbb{T}), \ldots, I^{g}(\mathbb{T})\right)$ with $I^{j}(\mathbb{T})=\sum_{n=1}^{\infty} I_{n}^{j} t_{n}$, and $I[z]=\left(I^{1}[z], \ldots, I^{g}[z]\right)$ with $I^{j}[z]=\sum_{n=1}^{\infty} I_{n}^{j} \frac{z^{n}}{n}$. For notations of $f(z), \varphi^{(n)}(z)$ and $I_{n}^{j}$ see Appendix $A$.

For simplicity $\Phi\left(z, \mathbb{T}, X_{c}\right)$ will simply be written as $\Phi(z, \mathbb{T})$ hereafter. Note that $I(\mathbb{T})$ is formal power series of $\mathbb{T}$, we consider $\Theta(I(\mathbb{T}) \mid \Omega)$ as the following formal Taylor series expansion:

$$
\Theta(I(\mathbb{T}) \mid \Omega)=\sum_{N=0}^{\infty} \frac{1}{N !}\left(I(\mathbb{T}) \frac{\partial}{\partial y}\right)^{N} \Theta(0 \mid \Omega) \in \mathbb{C}[[\mathbb{T}]],
$$

where $\frac{\partial}{\partial y}=\left(\frac{\partial}{\partial y^{1}}, \ldots, \frac{\partial}{\partial y^{g}}\right)$ with $\frac{\partial}{\partial y^{i}}$ denoting the derivation with respect to the $i$-th argument in $\Theta$.

Proof. We can show $\Phi(z, \mathbb{T}) \in \Lambda\left(X_{c}\right)$ by checking the periodicity as multiplicative function associated with $\mathscr{L}_{c}$ and the transformation property as a half-form. Suppose there is another $\Psi(z, \mathbb{T})(\neq 0) \in \Lambda\left(X_{c}\right)$, the ratio $\Psi / \Phi$ possesses neither an essential singularity nor a pole at $Q$ anymore. Furthermore, in the ratio $\Psi / \Phi$, the transformation factor as a form and the multiplicative factor are canceled out. Hence for the expansion of the ratio

$$
\frac{\Psi(z, \mathbb{T})}{\Phi(z, \mathbb{T})}=\frac{\sum_{N} \Psi_{N}(z) \mathbb{T}^{N}}{\sum_{M} \Phi_{M}(z) \mathbb{T}^{M}} \equiv \sum_{N} f_{N}(z) \mathbb{T}^{N},
$$

$f_{N}(z)$ is a meromorphic function on $R$ without poles at $Q$. We show that $f_{N}(z)$ is constant by induction with respect to $|N|=0,1,2, \ldots$ :

i) For $|N|=0$, i.e. $N=0=(0,0, \ldots)$, the pole divisor of $f_{0}(z)=\Psi_{0}(z) / \Phi_{0}(z)$ is at most $D=(\Theta(I[z]+c \mid \Omega))$, the zero divisor of $\Theta(I[z]+c \mid \Omega)$. From the assumption $\Theta(c \mid \Omega) \neq 0, D$ is nonspecial and $\operatorname{deg} D=g$. Thus $f_{0}(z) \in \mathscr{L}(D)=\mathbb{C} 1$.

ii) Assuming $f_{M}(z)=$ const for $|M|<|N|$, we have

$$
f_{N}(z)=\text { coefficient of } \mathbb{T}^{N} \text { in }\left[\Psi(z)-\Phi(z) \sum_{|M|<|N|} f_{M}(z) \mathbb{T}^{N}\right] / \Phi_{0}(z) .
$$

Since the pole divisor of $f_{N}(z)$ also divides $D$ (non-special), it follows just like i) that $f_{N}(z)=$ const. We then conclude

$$
\forall \Psi(z, \mathbb{T}) \in \Lambda\left(X_{c}\right): \frac{\Psi(z, \mathbb{T})}{\Phi(z, \mathbb{T})}=\sum_{N} C_{N} \mathbb{T}^{N} \in \mathbb{C}[[\mathbb{T}]],
$$

namely, $\Lambda\left(X_{c}\right)$ is a free $\mathbb{C}[[\mathbb{T}]]$-module of rank 1 . It works similarly for the conjugate sector.

Here it should be noted that $\Phi(z, \mathbb{T})$ and $\bar{\Phi}(z, \mathbb{T})$ given above are normalized as $w_{0}(\mathbb{T})=\bar{w}_{0}(\mathbb{T})=1$. We can then identify $\Phi(z, \mathbb{T})$ and $\bar{\Phi}(z, \mathbb{T})$ as the wave functions associated with $U\left(X_{c}\right) \in \mathrm{UGM}^{\phi}$. 
B) $\tau$-Function for $\hat{\mathscr{C}}_{g}(\tilde{\mathscr{J}})$

(5.5) Definition. ( $\tau$-function for $\hat{\mathscr{C}}_{g}(\tilde{\mathscr{J}})$ ) [I.M.O.; A-G.G.R.; V.]. Define $\tau: \widehat{\mathscr{C}}_{g}(\tilde{\mathscr{J}}) \rightarrow \mathscr{H}_{0} \times$ as

$$
\tau\left(\mathbb{T}, X_{c}\right)=e^{\frac{1}{2} q(\mathbb{T})} \Theta(I(\mathbb{T})+c \mid \Omega),
$$

where $q(\mathbb{T})=\sum_{n, m>0} q_{n m} t_{n} t_{m}$ (cf. Appendix A-1). We call this a $\tau$-function associated
with $\hat{\mathscr{C}}_{g}(\tilde{\mathscr{J}})$. (5.6) Theorem. For the $\tau$-function (5.5) the following diagram is commutative:

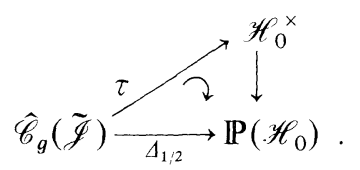

Proof. By direct calculations we obtain the following:

$$
\begin{aligned}
& \Phi\left(z, \mathbb{T}, X_{c}\right)=e^{-\xi(\mathbb{T}, z) \frac{\tau\left(\mathbb{T}+[z], X_{c}\right)}{\tau\left(\mathbb{T}, X_{c}\right)},} \\
& \bar{\Phi}\left(z, \mathbb{T}, X_{c}\right)=e^{\xi(\mathbb{T}, z)} \frac{\tau\left(\mathbb{T}-[z], X_{c}\right)}{\tau\left(\mathbb{T}, X_{c}\right)},
\end{aligned}
$$

which then implies that $\tau: \hat{\mathscr{C}}_{g}(\tilde{\mathscr{J}}) \rightarrow \mathscr{H}_{0}{ }^{\times}$is a lifting of $\Delta_{1 / 2}$.

(5.7) Remark. 1) In this way the fundamental problem (5.1) is solved affirmatively with the $\tau$-function given in (5.5). Define $\lambda_{1 / 2}$ is the line bundle associated with $\mathbb{C}^{*}$ bundle $\widetilde{\mathscr{C}}_{g}(\tilde{\mathscr{F}}) \rightarrow \hat{\mathscr{C}}_{g}(\tilde{\mathscr{F}})$, the $\tau\left(\mathbb{T}, X_{c}\right)$ gives a nowhere vanishing section of $\lambda_{1 / 2}$.

2) The vacuum amplitude $\tau\left(0, X_{c}\right)=\Theta(c \mid \Omega)$ depends only on $\tilde{\mathscr{J}}=\mathscr{T}_{g} \times \mathbb{C}^{g}$.

(5.8) So far we have no principle to eliminate the lifting ambiguity. From the point of view of the two-dimensional conformal field theory it is unimportant because the correlation functions depend only on the ratios of the $\tau$-function (or its derivative). On the other hand in string theory the lifting itself is a fundamental quantity and provides the integrand of the string amplitude. It should then possess a well-behaved automorphic property with respect to a modular transformation and good boundary behavior towards the boundary of $\mathscr{C}_{g}$ (stable curves [F.S.; F.; N.]). We thus discuss the modular transformation property of the $\tau$-function in the next paragraph.

\section{C) Modular Transformation Property of the $\tau$-Function}

(5.9) A modular transformation is induced by a change of the canonical homology basis $(\alpha, \beta)$, and is represented by a symplectic matrix $\gamma=\left(\begin{array}{ll}A & B \\ C & D\end{array}\right) \in S p(2 g, \mathbb{Z})$ as $\gamma\left(\begin{array}{l}\alpha \\ \beta\end{array}\right)=\left(\begin{array}{ll}D & C \\ B & A\end{array}\right)\left(\begin{array}{l}\alpha \\ \beta\end{array}\right)$. In particular $M_{\Delta}=\left\{\gamma \in M ; \operatorname{diag} C^{t} D \equiv \operatorname{diag} A^{t} B \equiv 0(\bmod 2)\right\}$ $\subset M$ is a subgroup of $M$ and keeps the Riemann constant invariant. Our $\tau$-function (5.5) has the following well-behaved automorphy under the modular transformation $M_{\Delta}$. 
(5.10) Theorem. For the t-function (5.5) we have

$$
\begin{gathered}
\tau\left(\mathbb{T}, \gamma\left(X_{c}\right)\right)=\varepsilon(\gamma) \operatorname{det}(C \Omega+D)^{1 / 2} \exp \left(\pi \sqrt{-1^{t}} c(C \Omega+D)^{-1} C(2 I(\mathbb{T})+c)\right) \tau\left(\mathbb{T}, X_{c}\right) \\
\text { for } \forall \gamma \in M_{\Delta},
\end{gathered}
$$

where $\varepsilon(\gamma)$ is a constant with $\varepsilon(\gamma)^{8}=1$ and depending only on $\gamma$.

Proof. The modular transformation property of the following quantities on a Riemann surface are given by

$$
\begin{aligned}
& \gamma(\Omega)=(A \Omega+B)(C \Omega+D)^{-1}, \\
& \gamma(I(\mathbb{T}))={ }^{t}(C \Omega+D)^{-1} I(\mathbb{T}), \\
& \gamma(q(\mathbb{T}))=-2 \pi \sqrt{-1} I(\mathbb{T})(C \Omega+D)^{-1} C I(\mathbb{T}), \\
& \gamma(c)={ }^{t}(C \Omega+D)^{-1} c .
\end{aligned}
$$

(See Appendix A for the notations.) Together with the transformation property of the $\Theta$-function [Mum. 1; A-G.M.V.] for $\gamma \in M_{\Delta}$ :

$$
\Theta\left({ }^{t}(C \Omega+D)^{-1} z \mid \gamma(\Omega)\right)=\varepsilon(\gamma) \operatorname{det}(C \Omega+D)^{1 / 2} e^{\pi \sqrt{-1^{t}} z(C \Omega+D)^{-1} z} \Theta(z \mid \Omega),
$$

we obtain the desired formula.

It should be noted that the $\tau$-function has a simpler automorphic factor than the $\Theta$-function itself (when $c=0$ ) because of the prefactor $\mathrm{e}^{\frac{1}{2} q(\mathbb{T})}$.

Similarly the $\tau$-function has the following well-behaved automorphy with respect to $c \in \mathbb{C}^{g}$ :

\section{Theorem.}

$$
\begin{gathered}
\tau\left(\mathbb{T}, X_{c+\Omega a+b}\right)=\exp \left(-2 \pi \sqrt{-1}\left(\frac{1}{2}{ }^{t} a \Omega a+{ }^{t} a(I(\mathbb{T})+c)\right)\right) \tau\left(\mathbb{T}, X_{c}\right), \\
\text { for } \forall a, b \in \mathbb{Z}^{g} .
\end{gathered}
$$

This is a direct consequence of the quasi-periodicity:

$$
\Theta(z+\Omega a+b \mid \Omega)=\exp \left(-2 \pi \sqrt{-1}\left(\frac{1}{2}^{t} a \Omega a+{ }^{t} a(z)\right)\right) \Theta(z \mid \Omega) .
$$

This automorphy turns out to be very important to characterize the $\tau$-function, which will be discussed in Sect. 7,D).

\section{D) Scattering Operator $S\left(X_{c}\right)$ [I.M.O.; A-G.G.R.; V.]}

(5.12) Before closing this section, we construct an operator $S\left(X_{c}\right)$ which generates the state vector $\left|X_{c}\right\rangle$ of the Fock space

$$
\left|X_{c}\right\rangle=S\left(X_{c}\right)|0\rangle\left\langle 0 \mid X_{c}\right\rangle, \quad\langle 0| S\left(X_{c}\right)=\langle 0|,
$$

where $X_{c}=\left(R,(\alpha, \beta), \mathscr{L}_{c}, u\right) \in \widehat{\mathscr{C}}_{g}(\tilde{\mathscr{J}})$ and we assume $\Theta(c \mid \Omega) \neq 0$. This $S\left(X_{c}\right)$ is very convenient in practical calculations (see Sect. 6). For example Wick's theorem in the calculation of various $N$-point functions can be easily verified by means of this operator. 
We expand the Szegö kernel [Fay; So.] of the line bundle $\mathscr{L}_{\Delta} \otimes \mathscr{L}_{\mathrm{c}}$ around $Q \in R$ as follows:

$$
S_{c}(z, w)=\frac{\Theta(I[z]-I[w]+c \mid \Omega)}{\Theta(c \mid \Omega) E(z, w)}=\frac{1}{z-w}+\sum_{\mu, v>0} C_{\mu v} z^{-\mu-1 / 2} w^{-v-1 / 2},
$$

then a basis, $\left\{\xi^{\mu}\right\}_{\mu \in \mathbb{Z} h}$ of $\mathscr{V}$, can be given by

$$
\begin{aligned}
& \xi^{\mu}=e^{\mu} \quad(\mu>0), \\
& \xi^{-\mu}=\frac{1}{2 \pi \sqrt{-1}} \int_{(x) \cdot z} d w w^{\mu-1 / 2} S_{c}(z, w)=e^{-\mu}+\sum_{v>0} C_{v \mu} e^{v} \quad(\mu>0),
\end{aligned}
$$

or equivalently in the matrix form

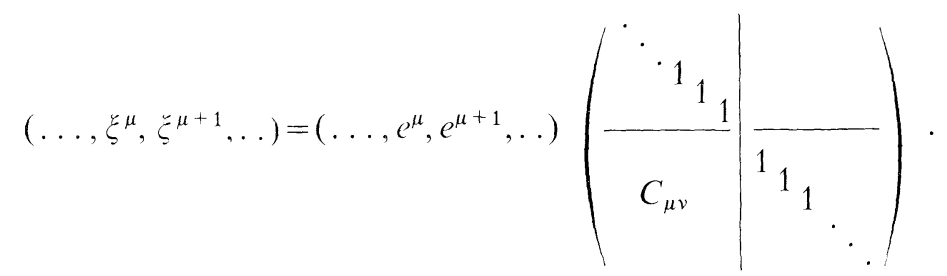

It is easy to check that $\xi^{-\mu}$ belongs to $U\left(X_{\mathrm{c}}\right)=\sqrt{d u} H^{0}\left(R, \mathscr{L}_{\Delta} \otimes \mathscr{L}_{\mathrm{c}}(* Q)\right) \in \mathrm{UGM}^{\phi}$ for $\mu>0$.

Define an operator $Q\left(X_{c}\right)$ as follows;

$$
\begin{aligned}
Q\left(X_{c}\right) & =\left(\frac{1}{2 \pi \sqrt{-1}}\right)^{2} \int_{\infty} d z \int_{\infty} d w\left\{S_{c}(z, w)-\frac{1}{z-w}\right\} \bar{\psi}(z) \psi(w) \\
& =\sum_{\mu \nu>0} C_{\mu \nu} \bar{\psi}_{-\mu} \psi_{-v} .
\end{aligned}
$$

Note that $Q\left(X_{c}\right)$ is constructed from creation operators only and thus belongs to $P_{-}$ and its exponential,

$$
S\left(X_{c}\right)=e^{-Q\left(X_{c}\right)}, \quad S\left(X_{c}\right)^{-1}=e^{Q\left(X_{c}\right)}
$$

are well-defined operators on $\mathscr{F}$.

\section{(5.13) Proposition.}

$$
\begin{aligned}
& \psi\left(z, X_{c}\right) \equiv S\left(X_{c}\right)^{-1} \psi(z) S\left(X_{c}\right)=\sum_{\mu \in \mathbb{Z} h} \psi_{\mu} \xi^{\mu}, \\
& \bar{\psi}\left(z, X_{c}\right) \equiv S\left(X_{c}\right)^{-1} \bar{\psi}(z) S\left(X_{c}\right)=\sum_{\mu \in \mathbb{Z} h} \bar{\psi}_{\mu} \bar{\xi}^{\mu},
\end{aligned}
$$

where $\bar{\xi}^{\mu}$ 's are the dual of $\xi^{\mu}:\left(\bar{\xi}^{\mu} \mid \xi^{\nu}\right)=\delta^{\mu+v, 0}$.

$$
\begin{aligned}
\left|X_{\mathrm{c}}\right\rangle & =S\left(X_{\mathrm{c}}\right)|0\rangle\left\langle 0 \mid X_{\mathrm{c}}\right\rangle, \\
\langle 0| & =\langle 0| S\left(X_{\mathrm{c}}\right) .
\end{aligned}
$$




\section{Action of Operators on the $\tau$-Function and Correlation Functions}

(6.0) Using the concrete expression of the $\tau$-function for $\hat{\mathscr{C}}_{g}(\tilde{\mathscr{J}})$ given in the previous section (5.5), we study the action of various (vertex, current, energymomentum) operators. We can derive $N$-point functions of Riemann surfaces of arbitrary genus (obtained recently by several authors [I.M.O.; A-G.G.R.]). Thanks to the fermion-boson correspondence, we can derive $N$-point functions in two different ways to obtain nontrivial identities [V.V.; E.O.2; Fay], which are closely related to the Schottky problem [Mum.3; v.G.; Sh.].

\section{A) Action of Vertex Operators}

(6.1) The vertex operators $V_{k}(z)(k \in \mathbb{Z})$ has been defined as follows (4.4):

$$
V_{k}(z)=\exp \left(\mathrm{k} \sum_{n=1}^{\infty} t_{n} z^{n}\right) e^{k t_{0}} z^{k \partial_{0}} \exp \left(-k \sum_{n=1}^{\infty} \frac{1}{n} z^{-n} \partial_{n}\right)
$$

Letting the vertex operators operate on the $\tau$-function, we obtain the following result.

(6.2) Theorem.

$$
\begin{aligned}
V_{k_{1}}\left(z_{1}\right) \ldots V_{k_{N}}\left(z_{N}\right) \tau\left(\mathbb{T}, X_{c}\right)= & \prod_{i=1}^{N} f\left(z_{i}\right)^{k_{2} K} e^{t_{0} K} \exp \left(\sum_{n=1}^{\infty} t_{n} \sum_{i=1}^{N} k_{i} \varphi^{(n)}\left(z_{i}\right)\right) \\
& \cdot \prod_{1 \leqq i<j \leqq N} E\left(z_{i}, z_{j}\right)^{k_{i} k_{J}} e^{\frac{1}{2} q(\mathbb{T})} \Theta\left(I(\mathbb{T})+\sum_{i=1}^{N} k_{i} I\left[z^{i}\right]+c \mid \Omega\right),
\end{aligned}
$$

where $K=\sum_{i=1}^{N} k_{i}\left(\right.$ total charge of operators $\left.V_{k_{1}}\left(z_{1}\right) \ldots V_{k_{N}}\left(z_{N}\right)\right)$.

See Appendix A for the notations. In the derivation of this formula we have used the following relation:

$$
\begin{aligned}
& V_{k}(z) V_{\ell}(w)=(z-w)^{k \ell} \circ V_{k}(z) V_{\ell}(w)_{\circ}^{\circ}, \\
& \left(\sum_{i=1}^{N} k_{i} x_{i}\right)^{2}=-\sum_{1 \leqq i<j \leqq N} k_{i} k_{j}\left(x_{i}-x_{j}\right)^{2}+K \sum_{i=1}^{N} k_{i} x_{i}^{2} .
\end{aligned}
$$

In particular for $N=1$ and $k_{i}= \pm 1$, we obtain the following:

\section{(6.3) Corollary.}

$$
\begin{aligned}
& \Psi\left(z, \mathbb{T}, X_{c}\right)=\frac{V_{-1}(z) \tau\left(\mathbb{T}, X_{c}\right)}{\tau\left(\mathbb{T}, X_{c}\right)} e^{t_{0}}=f(z) \exp \left(-\sum_{n=1}^{\infty} t_{n} \varphi^{(n)}(z)\right) \frac{\Theta(I(\mathbb{T})+I[z]+c \mid \Omega)}{\Theta(I(\mathbb{T})+c \mid \Omega)}, \\
& \bar{\Psi}\left(z, \mathbb{T}, X_{c}\right)=\frac{V_{1}(z) \tau\left(\mathbb{T}, X_{c}\right)}{\tau\left(\mathbb{T}, X_{c}\right)} e^{-t_{0}}=f(z) \exp \left(\sum_{n=1}^{\infty} t_{n} \varphi^{(n)}(z)\right) \frac{\Theta(I(\mathbb{T})-I[z]+c \mid \Omega)}{\Theta(I(\mathbb{T})+c \mid \Omega)} .
\end{aligned}
$$

These are just the BA-functions $\Phi(z, \mathbb{T})$ and $\bar{\Phi}(z, \mathbb{T})$ given in Theorem (5.4). 


\section{B) Action of Current Operators}

(6.4) The bosonized expression of the current operator has been given by

$$
J_{B}(z)=\sum_{n=1}^{\infty} n t_{n} z^{n-1}+\sum_{n=1}^{\infty} z^{-n-1} \partial_{n}
$$

in (4.5). When the current operator $J_{B}(z)$ passes through the bilinear factor $e^{\frac{1}{2} q(\mathbb{T})}$ of the $\tau$-function, it receives the following change;

$$
J_{B}(z) e^{\frac{1}{2} q(\mathbb{T})}=e^{\frac{1}{2} q(\mathbb{T})}\left\{\sum_{n=1}^{\infty} t_{n} \omega_{Q}^{(n)}(z)+\sum_{n=1}^{\infty} z^{-n-1} \partial_{n}\right\} \equiv e^{\frac{1}{2} q(\mathbb{T})} \hat{J}_{B}(z) .
$$

Note that $\hat{J}_{B}(z)=\hat{J}_{B}(z, X)$ depends holomorphically on the data $\pi\left(X_{c}\right)=X$ $=(R,(\alpha, \beta), Q, u) \in \hat{\mathscr{C}}_{g}$.

\section{(6.5) Proposition.}

$$
J_{B}\left(z_{1}\right) \ldots J_{B}\left(z_{N}\right) \tau\left(\mathbb{T}, X_{c}\right)=\sum_{\text {comb. }}\left(\prod_{\text {pairs }} \widehat{\hat{J}_{B}\left(z_{i}\right)} \hat{J}_{B}\left(z_{j}\right)\right)\left(\prod _ { \text { rests } } \longdiv { \hat { J } _ { B } ( z _ { k } ) ) } \tau ( \mathbb { T } , X ),\right.
$$

where

$$
\begin{aligned}
& \widehat{J}_{B}(z) \hat{J}_{B}(w)=\omega(z, w) \\
& \hat{J}_{B}(z) \tau\left(\mathbb{T}, X_{c}\right)=\left\{\sum_{n=1}^{\infty} t_{n} \omega_{Q}^{(n)}(z)+\sum_{i=1}^{g} \omega^{i}(z) \frac{\partial}{\partial y^{l}}\right\} \tau\left(\mathbb{I}, X_{c}\right),
\end{aligned}
$$

with

$$
\frac{\partial}{\partial y^{i}} \tau\left(\mathbb{T}, X_{c}\right)=e^{\frac{1}{2} q(\mathbb{T})} \frac{\partial}{\partial y^{i}} \Theta(I(\mathbb{T})+c \mid \Omega),
$$

and $\sum_{\text {comb }}$ is a summation over all the combinations of dividing $(1,2, \ldots N)$ into pairs $\left(i_{1} i_{2}\right),\left(i_{3} i_{4}\right), \ldots\left(i_{2 k-1} i_{2 k}\right)$ and the rest of the numbers $\left(i_{2 k+1}, \ldots, i_{N}\right)$.

\section{(6.6) Corollary.}

$$
J_{B}(z) \tau\left(\mathbb{T}, X_{c}\right)=\left\{\sum_{n=1}^{\infty} t_{n} \omega_{Q}^{(n)}(z)+\sum_{i=1}^{g} \omega^{i}(z) \frac{\partial}{\partial c^{i}}\right\} \tau\left(\mathbb{T}, X_{c}\right) .
$$

In order to see the meaning of this equality, we make use of the following definition.

(6.7) Definition. For $n \in \mathbb{Z}$ with $n>0$ we define holomorphic vector field $v^{(n)}$ on $\mathbb{P}\left(\hat{\mathscr{X}}_{g}^{(0)}\right)$ by

$$
v_{\tilde{X}}^{(n)}=\theta_{\tilde{X}}\left(\varphi^{(n)}(z, X)\right) \in T_{\tilde{X}} \mathbb{P}\left(\hat{\mathscr{X}}_{g}^{(0)}\right) \quad \tilde{X} \in \hat{\mathscr{X}}_{g}^{(0)}
$$

Note that $\varphi^{(n)}(z, X)=\int^{z} \omega_{Q}^{(n)}(X)$ depends holomorphically on the data $X=\pi(\tilde{X}) \in \hat{\mathscr{C}}_{g}$ (cf. Appendix A). 
(6.8) Theorem. Consider the diagram below

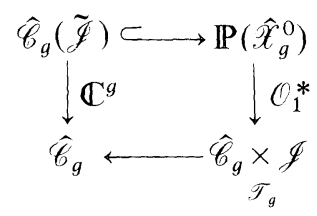

1) $v^{(n)}$ is a holomorphic vector field on $\mathbb{P}\left(\hat{\mathscr{X}}_{g}^{(0)}\right)$ along the image of $\hat{\mathscr{C}}_{g}(\tilde{\mathscr{J}})$ and thus induces a vector field on $\hat{\mathscr{C}}_{g}(\tilde{\mathscr{J}})$.

2) The induced vector field $\nu^{(n)}$ on $\hat{\mathscr{C}}_{g}(\tilde{\mathscr{J}})$ is tangential to the fibre of the fibering $\pi: \hat{\mathscr{C}}_{g}(\tilde{\mathscr{J}}) \rightarrow \hat{\mathscr{C}}_{g}$ and the action on this direction is given by

$$
v_{X_{c}}^{(n)}=\sum_{i=1}^{g} \frac{1}{2 \pi \sqrt{-1}} \int_{\beta_{\imath}} \omega_{Q}^{(n)}(X) \frac{\partial}{\partial c^{i}} .
$$

We can see that $J_{B}(z)$ is an operator which generates infinitesimal deformations of line bundles.

C) Action of Energy Momentum Tensor

(6.9) Since the bosonized expression of the energy momentum tensor is given by

$$
T_{B}(z)=\frac{1}{2} \circ J_{B}(z) J_{B}(z) \circ=\lim _{w \rightarrow z} \frac{1}{2}\left(J_{B}(z) J_{B}(w)-\frac{1}{(z-w)^{2}}\right)
$$

in (4.5), we can easily obtain the action of $T_{B}(z)$ by using Proposition (6.5).

(6.10) Theorem.

$$
\begin{aligned}
T_{B}(z) \tau\left(\mathbb{T}, X_{c}\right)= & {\left[\frac{1}{2} \sum_{n, m=1}^{\infty} t_{n} t_{m} \omega_{Q}^{(n)}(z) \omega_{Q}^{(m)}(z)+\sum_{n=1}^{\infty} \sum_{i=1}^{g} t_{n} \omega_{Q}^{(n)}(z) \omega^{i}(z) \frac{\partial}{\partial y^{i}}\right.} \\
& \left.+\sum_{i, j=1}^{g} \omega^{i}(z) \omega^{j}(z) \frac{\partial^{2}}{\partial y^{i} \partial y^{j}}-\frac{1}{12} S(z, X)\right] \tau\left(\mathbb{T}, X_{c}\right),
\end{aligned}
$$

where $S(z, X)$ is a projective connection (Appendix A-5).

We obtain the vacuum expectation value of the energy-momentum tensor

$$
\langle T(z)\rangle_{X_{c}}=\frac{\left\langle 0|T(z)| X_{c}\right\rangle}{\left\langle 0 \mid X_{c}\right\rangle} .
$$

(6.11) Proposition [A.W.; So.; I].

$$
\langle T(z)\rangle_{X_{c}}=\frac{1}{2} \frac{1}{\Theta(c \mid \Omega)} \sum_{i, j=1}^{g} \omega^{i}(z) \omega^{j}(z) \frac{\partial^{2}}{\partial y^{i} \partial y^{j}} \Theta(c \mid \Omega)-\frac{1}{12} S(z, X) .
$$

Using the heat equation of the $\Theta$-function:

$$
\frac{\partial^{2}}{\partial y^{i} \partial y^{j}} \Theta(y \mid \Omega)=4 \pi \frac{\partial}{\partial \Omega_{i j}} \Theta(y \mid \Omega),
$$


we can recognize that the energy momentum tensor induces infinitesimal deformations of moduli [So.; E.O.1].

\section{D) N-Point Functions [Ku.; E.O.2; A-G.G.R.]}

In the following we fix the data set $X_{c}=\left(R, Q,(\alpha, \beta), \mathscr{L}_{\Delta} \otimes \mathscr{L}_{c}, u\right)$ such that $\tau\left(0, X_{c}\right)$ $=\Theta(c \mid \Omega) \neq 0$ and denote $\left|X_{c}\right\rangle=B^{-1} \tau\left(\mathbb{T}, X_{c}\right) \in \mathscr{F}$.

(6.12) Definition. The $N$-point function of operators $\mathcal{O}_{1}\left(z_{1}\right), \ldots, \mathcal{O}_{N}\left(z_{N}\right)$ on a state $\left|X_{c}\right\rangle \in \mathscr{F}$ is defined as follows:

$$
\left\langle\mathcal{O}_{1}\left(z_{1}\right), \ldots, \mathcal{O}_{N}\left(z_{N}\right)\right\rangle_{X_{c}}=\frac{\left\langle 0\left|\mathcal{O}_{1}\left(z_{1}\right), \ldots, \mathcal{O}_{N}\left(z_{N}\right)\right| X_{c}\right\rangle}{\left\langle 0 \mid X_{c}\right\rangle} .
$$

Since we assume $\Theta(c \mid \Omega) \neq 0$, we can represent $\left|X_{c}\right\rangle \in \mathscr{F}$ as

$$
\left|X_{c}\right\rangle=S\left(X_{c}\right)|0\rangle\left\langle 0 \mid X_{c}\right\rangle
$$

hence the $N$-point function can be expressed as follows:

$$
\left\langle\mathcal{O}_{1}\left(z_{1}\right), \ldots, \mathcal{O}_{N}\left(z_{N}\right)\right\rangle_{X_{c}}=\left\langle 0\left|\mathcal{O}_{1}\left(z_{1}, X_{c}\right), \ldots, \mathcal{O}_{N}\left(z_{N}, X_{c}\right)\right| 0\right\rangle,
$$

where

$$
\mathcal{O}\left(z, X_{c}\right)=S\left(X_{c}\right)^{-1} \mathcal{O}(z) S\left(X_{c}\right)
$$

is a field operator corresponding to the Heisenberg picture.

On the other hand we can express the $N$-point function in the bosonized form:

$$
\left\langle\mathcal{O}_{1}\left(z_{1}\right), \ldots, \mathcal{O}_{N}\left(z_{N}\right)\right\rangle_{X_{c}}=\left.\frac{\mathcal{O}_{1 B}\left(z_{1}\right) \ldots \mathcal{O}_{N B}\left(z_{N}\right) \tau(\mathbb{T}, X)}{\tau\left(\mathbb{T}, X_{c}\right)}\right|_{\mathbb{T}=\mathbb{0}} .
$$

In the following we calculate the $N$-point functions of $\psi, \bar{\psi}$ 's and $J$ 's in two different ways given above.

(6.13) The fermion $2 N$-point function is obtained by putting $k_{i}= \pm 1$ in the formula (6.2):

$$
\begin{aligned}
& \left\langle\psi\left(z_{1}\right), \ldots, \psi\left(z_{N}\right) \bar{\psi}\left(w_{N}\right), \ldots, \bar{\psi}\left(w_{1}\right)\right\rangle_{X_{c}} \\
& =\frac{\prod_{i<j} E\left(z_{i}, z_{j}\right) \prod_{i<j} E\left(w_{j}, w_{i}\right)}{\prod_{i<j} E\left(z_{i}, w_{j}\right)} \frac{\Theta\left(\sum_{i=1}^{N} I\left[z_{i}\right]-\sum_{i=1}^{N} I\left[w_{i}\right]+c \mid \Omega\right)}{\Theta(c \mid \Omega)},
\end{aligned}
$$

while in the fermionic representation

$$
\left\langle\psi\left(z_{1}\right), \ldots, \psi\left(z_{N}\right) \bar{\psi}\left(w_{N}\right), \ldots, \bar{\psi}\left(w_{1}\right)\right\rangle_{X_{c}}=\operatorname{det}_{N \times N}\left\langle\psi\left(z_{i}\right) \bar{\psi}\left(w_{j}\right)\right\rangle_{X_{c}}
$$

and

$$
\langle\psi(z) \bar{\psi}(w)\rangle_{X_{c}}=\sum_{\mu>0} \xi^{\mu}(z) \bar{\xi}_{\mu}(w)=S(z, w)
$$


where

$$
S(z, w)=\frac{\Theta(I[z]-I[w]+c \mid \Omega)}{\Theta(c \mid \Omega) E(z, w)}
$$

is the Szegö kernel.

Identifying these two expressions, we obtain the following formula:

(6.14) Proposition (Fay's trisecant formula) [Fay; V.V.; E.O.1].

$$
\operatorname{det}_{N \times N} S\left(z_{i}, w_{j}\right)=\frac{\prod_{i<j} E\left(z_{i}, z_{j}\right) \prod_{i<j} E\left(w_{j}, w_{i}\right)}{\prod_{i, j} E\left(z_{i}, w_{j}\right)} \frac{\Theta\left(\sum_{i=1}^{N} I\left[z_{i}\right]-\sum_{i=1}^{N} I\left[w_{i}\right]+c \mid \Omega\right)}{\Theta(c \mid \Omega)} .
$$

The bosonic representation of current $N$-point functions can be obtained from (6.5):

(6.15) Proposition.

$$
\begin{aligned}
& \left\langle J\left(z_{1}\right), \ldots, J\left(z_{N}\right)\right\rangle_{X_{c}} \\
& \quad=\frac{1}{\Theta(c \mid \Omega)} \sum_{\text {comb. }}\left(\prod_{\text {pairs }} \omega\left(z_{i}, z_{j}\right)\right)\left(\prod_{\text {rests }}\left(\sum_{i=1}^{g} \omega^{i}\left(z_{k}\right) \frac{\partial}{\partial y^{i}}\right)\right) \Theta(c \mid \Omega) .
\end{aligned}
$$

On the other hand, using the fermionic representation of $J\left(z, X_{c}\right)$, we obtain

$$
\begin{aligned}
J\left(z, X_{c}\right) & =S\left(X_{c}\right)^{-1}\left\{\lim _{w \rightarrow z}\left(\bar{\psi}(z) \psi(w)-\frac{1}{z-w}\right)\right\} S\left(X_{c}\right) \\
& =\lim _{w \rightarrow z}\left\{\psi\left(z, X_{c}\right) \bar{\psi}\left(w, X_{c}\right)-\frac{1}{z-w}\right\},
\end{aligned}
$$

and taking the vacuum expectation value $\langle 0|\ldots| 0\rangle$, we obtain the following relations:

(6.16) Proposition.

where

$$
\left\langle J\left(z_{1}\right) \ldots J\left(z_{N}\right)\right\rangle_{X_{c}}=\operatorname{det}_{N \times N} S_{c}^{\text {reg }}\left(z_{i}, z_{j}\right),
$$

$$
\begin{gathered}
S_{c}^{\mathrm{reg}}(z, z)=\lim _{w \rightarrow z}\left\{S_{c}(z, w)-\frac{1}{z-w}\right\}=\frac{1}{\Theta(c \mid \Omega)} \sum_{i=1}^{g} \omega^{i}(z) \frac{\partial}{\partial y^{i}} \Theta(c \mid \Omega), \\
S_{c}^{\mathrm{reg}}\left(z_{i}, z_{j}\right)=S_{c}\left(z_{i}, z_{j}\right) \quad(i \neq j) .
\end{gathered}
$$

By equating these two expressions (6.15) and (6.16), we can derive various identies obtained by Fay. In order to get explicit expressions it is convenient to use the expression of the connected part of $N$-point functions;

$$
\left.\left\langle J\left(z_{1}\right) \ldots J\left(z_{N}\right)\right\rangle_{X_{c}}^{\text {conn }} \equiv J_{B}\left(z_{1}\right) \ldots J_{B}\left(z_{N}\right) \log \tau\left(\mathbb{T}, X_{c}\right)\right|_{\mathbb{T}=\mathbb{D}} .
$$


(6.17) Proposition. (1) Bosonic representation:

$$
\begin{aligned}
\left\langle J\left(z_{1}\right) \ldots J\left(z_{N}\right)\right\rangle_{X_{\mathrm{c}}}^{\text {conn. }}= & \sum_{i_{1} \ldots \ldots i_{N}}^{g} \frac{\partial^{N} \log \Theta(c \mid \Omega)}{\partial y^{i_{1}} \ldots \partial y^{i_{N}}} \omega^{i_{1}}\left(z_{1}\right) \ldots \omega^{i_{N}}\left(z_{N}\right) \\
& +\delta_{N, 2} \omega\left(z_{1}, z_{2}\right),
\end{aligned}
$$

(2) fermionic representation:

$$
\left\langle J\left(z_{1}\right) \ldots J\left(z_{N}\right)\right\rangle_{X_{c}}^{\text {conn. }}=-\sum_{\Gamma}\left(\prod_{\ell \in \Gamma} S_{\ell}\right),
$$

where $\Gamma$ is a connected oriented loop which passes through the points $z_{1}, z_{2}, \ldots, z_{N}$ once and for $\ell=\left(z_{i}, z_{j}\right) \in \Gamma: S_{\ell}=S_{c}\left(z_{i}, z_{j}\right)$.

(6.18) Proposition (Addition Formula).

$$
\sum_{i_{1} \ldots, i_{N}}^{g} \frac{\partial^{N} \log \Theta(c \mid \Omega)}{\partial y^{i_{1}} \ldots \partial y^{i_{N}}}-\omega^{i_{1}}\left(z_{1}\right) \ldots \omega^{i_{N}}\left(z_{N}\right)+\delta_{N, 2} \omega\left(z_{1}, z_{2}\right)=-\sum_{\Gamma}\left(\prod_{\ell \in \Gamma} S_{\ell}\right) .
$$

The following are special cases of this formula for $N=2$ and $N=4$ :

(6.19) Corollary [Fay].

1) $\omega\left(z_{1}, z_{2}\right)+\sum_{i j=1}^{g} \omega^{i}\left(z_{1}\right) \omega^{j}\left(z_{2}\right) \frac{\partial^{2}}{\partial y^{i} \partial y^{j}} \log \Theta(c \mid \Omega)=\left(S_{c}\left(z_{1}, z_{2}\right)\right)^{2}$,

2) $\sum_{i j k \ell=1}^{g} \omega^{i}\left(z_{1}\right) \omega^{j}\left(z_{2}\right) \omega^{k}\left(z_{3}\right) \omega^{\ell}\left(z_{4}\right) \frac{\partial^{4}}{\partial y^{i} \partial y^{j} \partial y^{k} \partial y^{\ell}} \log \Theta(c \mid \Omega)$

$$
=-\{S(1234)+S(1342)+S(1423)+S(1432)+S(1243)+S(1324)\},
$$

where $S(i j k \ell)=S_{c}\left(z_{i}, z_{j}\right) S_{c}\left(z_{j}, z_{k}\right) S_{c}\left(z_{k}, z_{\ell}\right) S_{c}\left(z_{\ell}, z_{i}\right)$ (see Fig. 2).
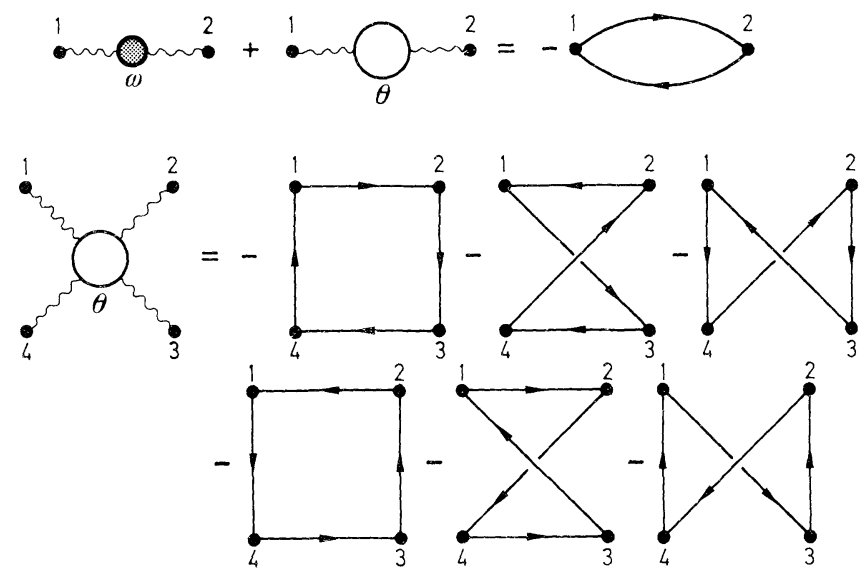

Fig. 2 


\section{Fundamental Equations}

(7.0) In this section we derive differential equations for the $\tau$-function $\tau\left(\mathbb{T}, X_{c}\right)$ $=e^{\frac{1}{2} q(\mathbb{T})} \Theta(I(\mathbb{T})+c \mid \Omega)$, defined on $\hat{\mathscr{C}}_{g}(\tilde{\mathscr{J}})$. Throughout this section we write $\Phi_{B}\left(s_{1 / 2} \circ \ell\right)=B \Phi\left(s_{1 / 2} \circ \ell\right) B^{-1}$ simply as $\Phi_{B}(\ell)$ for $\ell \in \mathbb{G}$ [see (2.32), (4.4)].

\section{A) The Fundamental Equations}

(7.1) Theorem (Fundamental equations). The $\tau$-function satisfies the following three equations:

1) (Equation of motion)

$$
\left[\theta(\ell)+a\left(\ell, X_{c}\right)\right] \tau\left(\mathbb{T}, X_{c}\right)=\Phi_{B}(\ell) \tau\left(\mathbb{T}, X_{c}\right) \quad \text { for } \quad \forall \ell \in \mathbb{G},
$$

where $a\left(\ell, X_{c}\right)=-\frac{1}{12} \operatorname{Res}_{z=\infty} \ell(z) S(z, X)$;

2) (Gauge condition)

$$
\sum_{i=1}^{g} \frac{1}{2 \pi \sqrt{-1}} \int_{\beta_{i}} d \varphi \frac{\partial}{\partial c^{i}} \tau\left(\mathbb{T}, X_{c}\right)=\Phi_{B}(\varphi) \tau\left(\mathbb{T}, X_{c}\right) \quad \text { for } \quad \forall \varphi(z) \in \tilde{A}(X)
$$

3) (Hirota's bilinear equation)

$$
\sum_{j=0}^{\infty} P_{j}(-2 \mathbb{Y}) P_{j+1}\left(\tilde{\mathbb{D}}_{\mathbb{T}}\right) \exp \left(\sum_{\ell=1}^{\infty} y_{\ell} D_{t_{\ell}}\right) \tau\left(\mathbb{T}, X_{\mathfrak{c}}\right) \cdot \tau\left(\mathbb{T}, X_{\mathfrak{c}}\right)=0 .
$$

[See Appendix A) for the definitions of $A(X)$ and $\tilde{A}(X)$.]

Proof. The equation 3) is already given in Theorem (4.16) and equation 2) is a rewriting of the following:

$$
\vartheta^{(n)} \tau\left(\mathbb{T}, X_{c}\right)=\Phi_{B}\left(\varphi^{(n)}(z, X)\right) \tau\left(\mathbb{T}, X_{c}\right) \quad \text { for } \quad \forall n>0
$$

which can be derived from Theorem (6.8).

Lastly we show 1). In the following diagram,

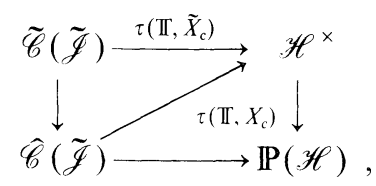

$\tau\left(\mathbb{T}, \tilde{X}_{c}\right)$ and $\tau\left(\mathbb{T}, X_{c}\right)$ are related as

$$
\tau\left(\mathbb{T}, \tilde{X}_{c}\right)=C\left(\tilde{X}_{c}\right) \tau\left(\mathbb{T}, X_{c}\right),
$$

where $C: \tilde{\mathscr{C}}_{g}(\tilde{\mathscr{J}}) \rightarrow \mathbb{C}^{*}$ is a holomorphic function satisfying $C\left(\lambda \tilde{X}_{c}\right)=\lambda C\left(\tilde{X}_{c}\right)$ $\left(\lambda \in \mathbb{C}^{*}\right)$. Letting $\tilde{\theta}(\ell), \ell \in \mathfrak{G}$, operate on $\tau\left(\mathbb{T}, \tilde{X}_{c}\right)$, we obtain the following relation from Proposition (3.35):

$$
\tilde{\theta}(\ell) \tau\left(\mathbb{T}, \tilde{X}_{c}\right)=\Phi_{B}(\ell) \tau\left(\mathbb{T}, \tilde{X}_{c}\right) .
$$


Then we obtain the desired equation. The term $a\left(\ell, X_{c}\right)$ is given by

$$
a\left(\ell, X_{c}\right)=\frac{1}{C\left(\tilde{X}_{c}\right)} \tilde{\theta}(\ell) C\left(\tilde{X}_{c}\right)
$$

and depends only on $X_{c}$. The explicit form will be given in the next paragraph.

The $\tau$-function being considered as a period map on $\widehat{\mathscr{C}}_{g}(\tilde{\mathscr{J}})$, the differential equation 1) is regarded as a defining equation of the Gauss-Manin connection, the equation of motion of the physical vacua in the interaction picture.

\section{B) Determination of the Anomaly Term a $\left(\ell, X_{c}\right)$}

In case $\ell \in \mathbf{G}_{0}$ the Eq. (7.1.1) can be viewed as the so-called "Ward-Takahashi identity". The term $a\left(\ell, X_{c}\right)$ corresponds to the anomaly term. In this view the equation in the following proposition can be identified as "Wess-Zumino consistency condition". Those principles symbolized by these terminologies which are familiar to physicists come very naturally into the formulation.

(7.2) Proposition (Consistency condition). For $\ell_{1}, \ell_{2} \in(5)$, we obtain the following relations:

$$
\theta\left(\ell_{1}\right) a\left(\ell_{2}, X_{c}\right)-\theta\left(\ell_{2}\right) a\left(\ell_{1}, X_{c}\right)+a\left(\left[\ell_{1}, \ell_{2}\right], X_{c}\right)=c\left(\ell_{1}, \ell_{2}\right),
$$

where

$$
c\left(\ell_{1}, \ell_{2}\right)=\frac{1}{12} \operatorname{Res}_{z=\infty} \ell_{1}^{\prime \prime \prime}(z) \ell_{2}(z)
$$

is the Schwinger term.

Proof. Consider the following equality:

$$
\left[\Phi_{B}\left(\ell_{1}\right), \Phi_{B}\left(\ell_{2}\right)\right]=\Phi_{B}\left(\left[\ell_{2}, \ell_{1}\right]\right)+c\left(\ell_{1}, \ell_{2}\right) \text {. }
$$

From the action of this operator on $\tau\left(\mathbb{T}, X_{c}\right)$ written in two ways, we obtain the desired result.

(7.3) We now determine the form of the anomaly term $a(\ell, X)$ corresponding to our $\tau$-function.

(7.4) Theorem. For the $\tau$-function the corresponding anomaly term is given by

$$
a\left(\ell, X_{c}\right)=-\frac{1}{12} \operatorname{Res}_{z=\infty} \ell(z) S(z, X),
$$

where $S(z, X)(d z)^{2}$ is the projective connection (see Appendix A-5).

Proof. First we show the following:

1) $a\left(\ell, X_{c}\right)=0$ for $\ell \in \mathfrak{G}_{1}$,

2) $a\left(\ell, X_{c}\right)=-\frac{1}{12} \operatorname{Res}_{z=\infty} \ell(z) S(z, X)$ for $\ell \in B(X)$,

3) $a\left(\ell, X_{c}\right)$ satisfies the consistency condition.

3) is Proposition (7.2) itself, while 2) can be obtained from Theorem (7.1-1) by taking $\ell \in B(X)$ since $\theta(\ell)=0$. 1) can be shown from the following representation of 
$\tau$ with the bosonization operator $B(4.2)$ :

$$
\tau\left(\mathbb{T}, X_{\mathrm{c}}\right)=\Theta(c \mid \Omega) B\left(\xi^{-1 / 2} \wedge \xi^{-3 / 2} \wedge \ldots\right),
$$

where $\left(\xi^{-1 / 2}, \xi^{-3 / 2}, \ldots\right)$ is a frame of $U\left(X_{c}\right)$ (cf. Sect. 5, D)).

From this expression we obtain the following for $\ell \in \mathfrak{G}_{1}$ :

$$
\begin{aligned}
\tau\left(\mathbb{T}, e^{\varepsilon \ell} X_{c}\right) & =\Theta(c \mid \Omega) B\left(e^{\varepsilon \ell}\left(\xi^{-1 / 2}\right) \wedge e^{\varepsilon \ell}\left(\xi^{-3 / 2}\right) \wedge \ldots\right) \\
\theta(\ell) \tau\left(\mathbb{T}, X_{c}\right) & =\Theta(c \mid \Omega) B\left(\sum_{\mu<0} \xi^{-1 / 2} \wedge \ldots \wedge \theta(\ell)\left(\xi^{\mu}\right) \wedge \ldots\right) \\
& =\Theta(c \mid \Omega) \Phi_{B}(\ell) B\left(\xi^{-1 / 2} \wedge \xi^{-3 / 2} \wedge \ldots\right) \\
& =\Phi_{B}(\ell) \tau\left(\mathbb{T}, X_{c}\right) .
\end{aligned}
$$

Hence $a\left(\ell, X_{c}\right)=0$ for $\ell \in \mathfrak{G}_{1}$.

Due to 1) $a\left(\ell, X_{c}\right)$ reduces to a linear function of $\ell$ on $\left(\mathfrak{5} / \mathfrak{G}_{1}\right.$. Fixing a sufficiently large $N$, we can take representatives of bases, $\widehat{\ell}_{n} \in \mathfrak{G}(n=0,1,2, \ldots)$, of $\mathfrak{5} / \mathfrak{b}_{1}$ in the following:

$$
\begin{aligned}
& \hat{\ell}_{n}=\left(z^{n+1}+\text { lower order terms }\right) \frac{d}{d z} \in B(X), \quad n \geqq N, \\
& \hat{\ell}_{n}=\left(a d \frac{d}{d z}\right)^{N-n} \hat{\ell}_{N}, \quad \frac{d}{d z} \in \mathfrak{G}_{1}, \quad 0 \leqq n<N .
\end{aligned}
$$

For $n \geqq N, a\left(\hat{\ell}_{n}, X_{c}\right)$ is given in 2$)$. For $0 \leqq n<N$, one obtains the following relations by induction on $n$ and with the consistency condition (7.2):

$$
\begin{aligned}
a\left(\hat{\ell}_{n-1}, X_{c}\right) & =a\left(\left[\frac{d}{d z}, \hat{\ell}_{n}\right], X_{c}\right)=-\theta\left(\frac{d}{d z}\right) a\left(\hat{\ell}_{n}, X_{c}\right) \\
& =\frac{1}{12} \operatorname{Res}_{z=\infty} \hat{\ell}_{n}(z) \frac{d}{d z} S\left(z, X_{c}\right)=-\frac{1}{12} \operatorname{Res}_{z=\infty} \hat{\ell}_{n-1} S\left(z, X_{c}\right) .
\end{aligned}
$$

In the derivation from the first line to the second we used the formula given in Appendix B. We thus obtain the desired result for all $n$.

\section{(7.5) Corollary.}

$$
\begin{aligned}
\theta(\ell) \tau\left(\mathbb{T}, X_{c}\right)= & \operatorname{Res}_{z=\infty} \ell(z)\left[\frac{1}{2} \sum_{n, m=1}^{\infty} t_{n} t_{m} \omega_{Q}^{(n)}(z) \omega_{Q}^{(m)}(z)\right. \\
& \left.+\sum_{n=1}^{\infty} \sum_{i=1}^{g} t_{n} \omega_{Q}^{(n)}(z) \omega^{i}(z) \frac{\partial}{\partial y^{i}}+\frac{1}{2} \sum_{i, j=1}^{g} \omega^{i}(z) \omega^{j}(z) \frac{\partial^{2}}{\partial y^{i} \partial y^{j}}\right] \tau\left(\mathbb{T}, X_{c}\right)
\end{aligned}
$$

for $\ell \in \mathfrak{5}$.

Proof. This equation is obtained from the result of Theorem (6.10) and (7.4). 
(7.6) Proposition. The projective connection $S(z, X)(d z)^{2}$ is transformed under the modular transformation $\gamma=\left(\begin{array}{ll}A & B \\ C & D\end{array}\right) \in \Gamma$ as follows:

$$
S(z, \gamma(X)) d z^{2}=S(z, X) d z^{2}+4 \pi \sqrt{-1}^{t} \omega(z)(C \Omega+D)^{-1} C \omega(z) .
$$

Proof. This relation is obtained from the expression of the projective connection:

$$
S(z, X) d z^{2}=-6\left[d_{z} d_{w} \log \Theta(I(z)-I(w) \mid \Omega)\right]_{w \rightarrow z},
$$

and the transformation formula of $\Theta$-function.

\section{C) Characterization of the $\tau$-Function}

(7.7) Main Theorem. For a holomorphic map $f: \hat{\mathscr{C}}_{g}(\tilde{\mathscr{J}}) \rightarrow \mathscr{H}^{\times}$the following equations determine $f$ uniquely up to a constant: $f\left(\mathbb{T}, X_{c}\right)=C \tau\left(\mathbb{T}, X_{c}\right)$ with $C \in \mathbb{C}$ :

1) $[\theta(\ell)+a(\ell, X)] f\left(\mathbb{T}, X_{c}\right)=\Phi_{B}(\ell) f\left(\mathbb{T}, X_{c}\right)$ for $\forall \ell \in \mathscr{G}$,

where

$$
\begin{aligned}
& a(\ell, X)=-\frac{1}{12} \operatorname{Res}_{z=\infty} \ell(z) S(z, X) \\
& \text { 2) } \frac{1}{2 \pi \sqrt{-1}} \sum_{i=1}^{g} \int_{\beta_{1}} d \varphi \frac{\partial}{\partial c^{i}} f\left(\mathbb{T}, X_{c}\right)=\Phi_{B}(\varphi) f\left(\mathbb{T}, X_{c}\right) \text { for } \forall \varphi(z) \in \tilde{A}(X) ; \\
& \text { 3) } f\left(\mathbb{T}, X_{c+\Omega a+b}\right)=\exp \left(-2 \pi \sqrt{-1}\left(\frac{1}{2}^{t} a \Omega a+{ }^{t} a(I(\mathbb{T})+c)\right)\right) f\left(\mathbb{T}, X_{c}\right) \\
& \text { for } a, b \in \mathbb{Z}^{g} .
\end{aligned}
$$

Proof. In equation 2) the left-hand side vanishes when $\varphi \in A(X)$, which specifies the form of $f\left(\mathbb{T}, X_{\mathrm{c}}\right)$ as

$$
f\left(\mathbb{T}, X_{c}\right)=e^{\frac{1}{2} q(\mathbb{T})} f_{0}\left(I(\mathbb{T}), X_{c}\right)
$$

with $f_{0}: \hat{\mathscr{C}}(\tilde{\mathscr{J}}) \rightarrow \mathbb{C}\left[\left[y_{1}, y_{2}, \ldots, y_{g}\right]\right]$.

Taking $\varphi^{(n)} \in \tilde{A}(X)$ (see Appendix A-2) in equation 2), we obtain equations

$$
\left(\frac{\partial}{\partial t_{n}}-\sum_{i=1}^{g} I_{n}^{i} \frac{\partial}{\partial c^{i}}\right) f_{0}=0, \quad n=1,2,3, \ldots
$$

which naturally lead to the following form for $f\left(\mathbb{T}, X_{c}\right)$ :

$$
f\left(\mathbb{T}, X_{c}\right)=e^{\frac{1}{2} q(\mathbb{T})} f_{0}(I(\mathbb{T})+c, X) .
$$

From the automorphy relation 3 ) with respect to the Jacobian parameter $c \in \mathbb{C}^{g}$, we obtain the proportionality of $f_{0}(I(\mathbb{T})+c, X)$ and $\Theta(I(\mathbb{T})+c \mid \Omega)$, i.e.

$$
f\left(\mathbb{T}, X_{c}\right)=C(X) \tau\left(\mathbb{T}, X_{c}\right) .
$$

The equations 1 ) shows that the factor $C(X)$ does not depend on $X$.

D) Principles to Determine the Lifting of $\Delta_{1 / 2}$

(7.8) We know already that our $\tau$-function has the following properties: 
1) Automorphy under $M_{\Delta} \ltimes \mathbb{Z}^{2 g}$

a) $\tau\left(\mathbb{T}, \gamma\left(X_{c}\right)\right)=\varepsilon(\gamma) \operatorname{det}(C \Omega+D)^{1 / 2}$ $\cdot \exp \left(\pi \sqrt{-1}^{t} c(C \Omega+D)^{-1} C(2 I(\mathbb{T})+c)\right) \tau\left(\mathbb{T}, X_{c}\right) \quad$ for $\quad \gamma \in M_{\Delta}$;

b) $\tau\left(\mathbb{T}, X_{c+\Omega a+b}\right)=\exp \left(-2 \pi \sqrt{-1}\left(\frac{1}{2}{ }^{t} a \Omega a+{ }^{t} a(I(\mathbb{T})+c)\right)\right) \tau\left(\mathbb{T}, X_{c}\right)$, $a, b \in \mathbb{Z}^{g}$

2) $\tau\left(0, X_{c}\right)$ depends only on $\mathscr{T}_{g} \times \mathbb{C}^{g}$.

(7.9) Theorem. Let $f$ be a lifting of $\Delta_{1 / 2}$ satisfying 1) and 2) in (7.8):

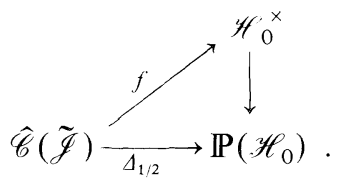

1) Then it can be written in the form:

$$
f\left(\mathbb{T}, X_{c}\right)=C\left(\pi\left(X_{c}\right)\right) \tau\left(\mathbb{T}, X_{c}\right),
$$

where $C: \mathscr{T} \rightarrow \mathbb{C}^{*}$ is holomorphic and $M_{\Delta}$-invariant and $\pi: \hat{\mathscr{C}}(\tilde{\mathscr{J}}) \rightarrow \mathscr{T}$.

2) Furthermore $C\left(\pi\left(X_{c}\right)\right)$ is constant if $g \geqq 3$.

Proof. 1) If we write $f\left(\mathbb{T}, X_{c}\right)=C\left(X_{c}\right) \tau\left(\mathbb{T}, X_{c}\right), C\left(X_{c}\right)$ does not depend on $c$ from the automorphy 1)-b). From this, 1)-a) and 2) we obtain the first half of the theorem.

2) If $g \geqq 3$ moreover, we know that $\mathscr{T} / M_{\Delta}$ has a compactification whose boundary is of codimension $>1$. Hence there is no holomorphic function on it (i.e. no $M_{\Delta}$-invariant holomorphic function on $\mathscr{T}$ ) besides constant.

\section{Appendix}

Here we list the notations and formulas on the theory of abelian functions which we use in this article. For details see for example [Fay; Mum.1]. We fix a Riemann surface $R$, a canonical basis $(\alpha, \beta)$ of $H_{1}(R, \mathbb{Z})$, a point $Q \in R$ and a (formal) local coordinate $z: z(Q)=\infty$. The whole data are denoted by $X=(R,(\alpha, \beta), Q, z)$. We set $X_{c}=\left(X, \mathscr{L}_{c}\right), c \in \mathbb{C}^{g}$ (cf. Sect. 2).

A) Abelian Differentials and the Green Function on Riemann Surfaces

1) Abelian differentials

(1st kind)

$$
\begin{gathered}
\omega^{i}=\omega^{i}(z) d z, \quad i=1 \ldots g, \\
\int_{\alpha_{2}} \omega^{j}=\delta_{i j}, \quad \int_{\beta_{2}} \omega^{j}=\Omega_{i j}, \\
\omega^{i}(z) d z=-d\left(\sum_{n>0} I_{n}^{i} \frac{z^{-n}}{n}\right) ;
\end{gathered}
$$


(2nd kind)

$$
\begin{gathered}
\omega_{Q}^{(n)}=\omega_{Q}^{(n)}(z) d z, \quad n=1,2 \ldots, \quad Q \in R \\
\int_{\alpha_{\imath}} \omega_{Q}^{(n)}=0, \quad \int_{\beta_{\imath}} \omega_{Q}^{(n)}=2 \pi \sqrt{-1} I_{n}^{i}, \\
\omega_{Q}^{(n)}(z) d z=d\left(z^{n}-\sum_{m>0} q_{n m} \frac{z^{-m}}{m}\right) .
\end{gathered}
$$

2) Green Functions

(Prime form): a holomorphic section (unique up to a constant) of the line bundle $\pi_{1}^{*} \mathscr{L}_{\Delta}^{-1} \otimes \pi_{2}^{*} \mathscr{L}_{\Delta}^{-1} \otimes \delta^{*}(\theta)$ over $R \times R$, with a simple zero only at $P=Q$ where $\pi_{1}, \pi_{2}, \delta$ are defined in the following diagram:

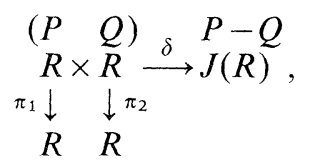

$$
\begin{aligned}
& E(P, Q)=E(z, w) \sqrt{d z}^{-1} \sqrt{d w}^{-1}=-E(Q, P), \\
& \partial_{z} \hat{\partial}_{w} \log \frac{E(z, w)}{z-w}=\sum_{n, m>0} q_{n m} z^{-n-1} w^{-m-1}, \\
& E\left(P+\alpha_{i}, Q\right)=E(P, Q), \\
& E\left(P+\beta_{i}, Q\right)=\exp \left(2 \pi \sqrt{-1}\left(\frac{\Omega_{i i}}{2}+\int_{Q}^{P} \omega^{i}\right)\right) E(P, Q) ;
\end{aligned}
$$

(multiplicative meromorphic half-form with a simple pole at $Q \in R$ )

$$
\begin{aligned}
& f(z) \sqrt{d z}=\left.\frac{\sqrt{d z} w}{E(z, w)}\right|_{w \rightarrow \infty} \\
& f\left(z+\alpha_{i}\right)=f(z), \\
& f\left(z+\beta_{i}\right)=\exp \left(-2 \pi \sqrt{-1}\left(\frac{\Omega_{i i}}{2}+\int_{Q}^{z} \omega^{i}\right) f(z)\right), \\
& f(z) \sqrt{d z}=\left(1+o\left(z^{-1}\right)\right) \sqrt{d z}
\end{aligned}
$$

(multivalued meromorphic functions with poles at $Q \in R$ )

$$
\begin{aligned}
& \varphi^{(0)}(z)=1 \\
& \varphi^{(n)}(z)=z^{n}-\sum_{n=1}^{\infty} q_{n m} \frac{z^{-m}}{m}=\int \omega_{Q}^{(n)}, \quad n=1,2,3 \ldots \\
& \varphi^{(n)}\left(z+\alpha_{i}\right)=\varphi^{(n)}(z) \\
& \varphi^{(n)}\left(z+\beta_{i}\right)=\varphi^{(n)}(z)+2 \pi \sqrt{-1} I_{n}^{i}
\end{aligned}
$$



If we set $\tilde{A}(X)=\sum_{n=0}^{\infty} \mathbb{C} \varphi^{(n)}(z) \subseteq K$ (a closed subspace), then the following sequence
is exact

$$
\begin{aligned}
0 \rightarrow A(X) \rightarrow \tilde{A}(X) & \rightarrow \mathbb{C}^{g} \rightarrow 0 \\
f & \rightarrow c_{i}=\frac{1}{2 \pi \sqrt{-1}} \int_{\beta_{i}} d f .
\end{aligned}
$$

(Fundamental normalized differential of the second kind)

$$
\begin{aligned}
& \omega(P, Q)=\omega(z, w) d z d w=d_{z} d_{w} \log E(z, w), \\
& \omega(z, w)=\frac{1}{(z-w)^{2}}+\sum_{n m>0} q_{n m} z^{-n-1} w^{-w-1} .
\end{aligned}
$$

(Szegö kernel)

$$
\begin{aligned}
& S_{c}(P, Q)=S_{c}(z, w) \sqrt{d z} \sqrt{d w}, \\
& S_{c}(z, w)=\frac{\Theta(I(z)-I(w)+c \mid \Omega)}{\Theta(c \mid \Omega) E(z, w)}=\frac{1}{z-w}+\sum_{\mu \nu>0} C_{\mu \nu} z^{-\mu-1 / 2} w^{-v-1 / 2},
\end{aligned}
$$

where $c \in \mathbb{C}^{g}$ with $\Theta(c \mid \Omega) \neq 0$.

3) The $\Theta$-function and the Abel-Jacobi map

( $\Theta$-function)

$$
\begin{aligned}
& \Theta\left[\begin{array}{l}
a \\
b
\end{array}\right](z \mid \Omega)=\sum_{n \in \mathbb{Z}^{g}} \exp \{\pi \sqrt{-1}(n+a) \Omega(n+a)+2 \pi \sqrt{-1}(n+a)(z+b)\} z \in \mathbb{C}^{g}, \\
& \Theta\left[\begin{array}{l}
a \\
b
\end{array}\right]\left(z+e_{i} \mid \Omega\right)=e^{2 \pi \sqrt{-1} a_{i}} \Theta\left(\begin{array}{l}
a \\
b
\end{array}\right)(z \mid \Omega), \\
& \Theta\left[\begin{array}{l}
a \\
b
\end{array}\right]\left(z+\Omega e_{i} \mid \Omega\right)=\exp \left\{-2 \pi \sqrt{-1}\left(\Omega_{i i} / 2+z_{i}+b_{i}\right)\right\} \Theta\left(\begin{array}{l}
a \\
b
\end{array}\right)(z \mid \Omega) .
\end{aligned}
$$

(Abel-Jacobi Map)

$I: \widetilde{R} \rightarrow \mathbb{C}^{g} ; \tilde{R}$ is the universal Abelian covering of $R$,

$$
I[z]=\left(\int_{Q}^{z} \omega^{i}\right)=\left(\sum_{n} I_{n}^{i} z^{-n}\right) .
$$

4) Modular transformation properties.

$$
\text { For } \begin{aligned}
\gamma=\left(\begin{array}{ll}
A & B \\
C & D
\end{array}\right) \in S p(2 g, \mathbb{Z})=M, \\
\begin{aligned}
\gamma\left(\begin{array}{l}
\alpha \\
\beta
\end{array}\right) & =\left(\begin{array}{ll}
D & C \\
B & A
\end{array}\right)\left(\begin{array}{l}
\alpha \\
\beta
\end{array}\right), \quad \gamma(\Omega)=(A \Omega+B)(C \Omega+D)^{-1}, \\
\gamma\left(\omega^{i}\right) & =\left({ }^{t}(C \Omega+D)^{-1}\right)_{i j} \omega^{j},
\end{aligned}
\end{aligned}
$$




$$
\begin{gathered}
\gamma\left(\omega_{Q}^{(n)}\right)=\omega_{Q}^{(n)}-\left((C \Omega+D)^{-1}\right)_{i j} C_{j k}\left(\int_{\beta_{\imath}} \omega_{Q}^{(n)}\right) \omega_{k} \\
\gamma\left(\begin{array}{l}
a \\
b
\end{array}\right)=\left(\begin{array}{cc}
D & -C \\
-B & A
\end{array}\right)\left(\begin{array}{l}
a \\
b
\end{array}\right)-\frac{1}{2} \operatorname{diag}\left(\begin{array}{l}
C^{t} D \\
A^{t} B
\end{array}\right), \\
\Theta\left[\gamma\left(\begin{array}{l}
a \\
b
\end{array}\right)\right]\left({ }^{t}(C \Omega+D)^{-1} z, \gamma(\Omega)\right) \\
=\varepsilon \operatorname{det}(C \Omega+D)^{1 / 2} \exp \left\{\pi \sqrt{-1} z(C \Omega+D)^{-1} C z\right\} \Theta\left[\begin{array}{l}
a \\
b
\end{array}\right](z \mid \Omega),
\end{gathered}
$$

where $\quad \varepsilon=\varepsilon(\gamma)$ with $\varepsilon^{8}=1$.

5) Projective connection

$$
\begin{aligned}
& S(P)=S(z) d z^{2}=-6 \lim _{w \rightarrow z} d_{z} d_{w} \log \frac{E(z, w)}{z-w} \\
& \omega(z, w)=\frac{1}{(z-w)^{2}}+\frac{1}{6} S(z)+\text { higher order terms } \\
& S(w) d w^{2}=S(z) d z^{2}+\{w, z\} d z^{2},
\end{aligned}
$$

where

$$
\{w, z\}=\frac{w^{\prime \prime \prime}}{w^{\prime}}-\frac{3}{2}\left(\frac{w^{\prime \prime}}{w^{\prime}}\right)^{2} \quad(\text { Schwarzian derivative })
$$

with $^{\prime}=\frac{d}{d z}$.

\section{B) Transformation Property and $\theta(\ell)$-Derivatives}

For $\ell=\ell(z) \frac{d}{d z} \in \mathfrak{G}_{0}, \theta(\ell)$ is essentially a generator of coordinate transformation which fixes $z(Q)=\infty$ :

$$
z \rightarrow w=e^{\varepsilon \ell} z=\left(1+\varepsilon \ell(z) \frac{d}{d z}\right) z=z+\varepsilon \ell(z) .
$$

We can thus calculate $\theta(\ell)$-derivatives of geometrical objects from the data of their transformation properties.

1) Let $f(P)$ be a $j$-form on $R$ and define $f(z, X): \widehat{\mathscr{C}}_{g} \rightarrow \mathbb{C}\left(\left(z^{-1}\right)\right)$ by $f(P)=f(z, X)(d z)^{j}$ for $X=(R, Q, z)$. We have the following relation:

$$
f\left(z, e^{\varepsilon t} X\right)(d z)^{j}=f(w, X)(d w)^{j} .
$$

On the other hand the transformation property as a $j$-form implies

$$
f(w, X)(d w)^{J}=f(z, X)(d z)^{j}+\varepsilon\left\{\ell(z) \frac{d}{d z}+j \ell^{\prime}(z)\right\} f(z, X)(d z)^{j} .
$$


It then follows that

$$
\theta(\ell) f(z, X)=\left.\frac{d}{d \varepsilon} f\left(z, e^{\varepsilon \ell} X\right)\right|_{\varepsilon=0}=\left\{\ell(z) \frac{d}{d z}=j \ell^{\prime}(z)\right\} f(z, X) .
$$

2) Let $S(P)$ be a projective connection on $R$ and define $S(z, X): \widehat{\mathscr{C}}_{g} \rightarrow \mathbb{C}\left(\left(z^{-1}\right)\right)$ by $S(P)=S(z, X)(d z)^{2}$, then just like 1) we have

$$
S\left(z, e^{\varepsilon t} X\right)(d z)^{2}=S(w, X)(d w)^{2}-\{w(z), z\}(d z)^{2}
$$

which lead to the following:

$$
\theta(\ell) S(z, X)=\left\{\ell(z) \frac{d}{d z}+2 \ell^{\prime}(z)\right\} S(z, X)-\ell^{\prime \prime \prime}(z) .
$$

\section{References}

[A-G.M.V] Alvarez-Gaumé, L., Moore, G., Vafa, C.: Theta functions, modular invariance, and strings. Commun. Math. Phys. 106, 1-40 (1986)

[A-G.M.N.V.B.] Alvarez-Gaumé, L., Moore, G., Nelson, P., Vafa, C., Bost, J.B. : Bosonization in arbitrary genus. Phys. Lett. 178B, 41 (1986)

Alvarez-Gaumé, L., Bost, J.-B., Moore, G., Nelson, Ph., Vafa, C.: Bosonization on higher genus Riemann surfaces. Commun. Math. Phys. 112, 503 (1987)

[A-G.G.R.] Alvarez-Gaumé, L., Gomez, C., Reina, C.: Loop groups, grassmanians and string theory. Phys. Lett. 190 B, 55 (1987)

[A.W.] Alvarez, O., Windey, P.: The energy momentum tensor as geometrical datum, LBL preprint, UCB-PTH-86/36, LBL-22558

[Ar.] Arakelov, S.: An intersection theory for divisors on an arithmetic surface. Izv. Akad. Nauk. SSSR Ser. Mat. 38 (1974) [= Math. USSR Izv. 8, 1167 (1974)]

[B.K.] Belavin, A.A., Knizhnik, V.G.: Complex geometry and theory of quantum string. Sov. Phys. JETP 64, 214 (1986)

[B.M.] Beilinson, A.A., Manin, Yu.I.: The Mumford form and the Polyakov measure in string theory. Commun. Math. Phys. 107, 359 (1986)

[B.M.S.] Beilinson, A.A., Manin, Yu.I., Shechtman, Y.A.: Localization of the Virasoro and Neveu-Schwartz algebra. Moscow preprint (1986)

[B.P.Z.] Belavin, A.A., Polyakov, A.M., Zamolodchikov, A.B.: Infinite conformal symmetry in two-dimensional quantum field theory. Nucl. Phys. B241, 333 (1984)

[B.R.] Bernshtein, I.N., Rozenfel'd, B.I.: Homogeneous spaces of infinitedimensional Lie algebra and characteristic classes of foliations. Russ. Math. Surv. 107, 28 (1973)

[B.Ra.] Bowick, M.J., Rajeev, S.G. : String theory as the Kähler geometry of loop space. Phys. Rev. Lett. 58, 535 (1987)

[D.J.K.M.] Date, E., Jimbo, M., Kashiwara, M., Miwa, T.: Transformation groups for soliton equations. In: Procs. of RIMS Symposium on Non-Linear Integrable Systems-Classical Theory and Quantum Theory, Kyoto, Japan. Jimbo, M., Miwa, T. (eds.). Singapore: World Science 1983

[D.M.] Deligne, P., Mumford, D.: The irreducibility of the space of curves of given genus. Publ. Math. I.H.E.S. 36, 75 (1969) 
[D.]

[D.S.]

[E.O.1]

[E.O.2]

[Fal.]

[F.K.]

[Fay]

[F.G.Z.]

[F.S.]

[Fr.]

[v.G.]

[I.M.O.]

[I.]

$[\mathrm{Kn}$.

[Ko.]

[K.N.]

[Kr.]

[Mul.]

[Mum.1]

[Mum.2]

[Mum.3]

[N.]

[P.K.]

[P.]

[Sa.]

Dubrovin, B.A. : Theta functions and non-linear equations. Usp. Mat. Nauk 36 (2), 11 (1981)

Dugan, M., Sonoda, H.: Functional determinants on Riemann surfaces. LBL preprint, LBL-22776, 1986

Eguchi, T., Ooguri, H.: Conformal and current algebras on a general Riemann surface. Nucl. Phys. B282, 308 [1987)

Eguchi, T., Ooguri, H.: Chiral bosonization on a Riemann surface. Phys. Lett. 187 B, 127 (1987)

Faltings, G.: Calculus on arithmetic surfaces. Ann. Math. 119, 387 (1984)

Farkas, H.M., Kra, I.: Riemann surfaces. Berlin, Heidelberg, New York: Springer 1980

Fay, J.: Theta functions on Riemann surfaces. Lecture Notes in Mathematics, Vol. 352. Berlin, Heidelberg, New York: Springer 1973

Frenkel, I.B., Garland, H., Zuckerman, G.J.: Semi-infinite cohomology and string theory. Proc. Nat. Acad. Sci. USA 83, 8442 (1986)

Friedan, D., Shenker, S.: The analytic geometry of two-dimensional conformal field theory. Nucl. Phys. B281, 509 (1987)

Friedan, D. : A new formulation of string theory, Second Nobel Symposium on Elementary Particle Physics, presented at Marstrand, Sweden (1986) and Symposium on Geometry and Topology in Field Theory, Espoo, Finland (1986)

van der Geer: Schottky's problem. (Proc.) Arbeitstagung Bonn. Lecture Notes in Mathematics, Vol. 1111. Berlin, Heidelberg, New York: Springer 1984

Hirota, R.: Direct method in soliton theory, Solitons, 157. Bullough, R.K., Caudrey, P.J. (eds.). Berlin, Heidelberg, New York: Springer 1980

Ishibashi, N., Matsuo, Y., Ooguri, H.: Soliton equations and free fermions on Riemann surfaces. Mod. Phys. Lett. A2, 119 (1987)

Ivanov, B.V.: On the quantum topology of strings. Phys. Lett. 189 B, 39 (1987)

Knizhnik, V.G.: Analytic fields on Riemann surfaces. Phys. Lett. 180B, 247 (1986)

Kodaira, K.: Complex manifolds and deformations of complex structure. Berlin, Heidelberg, New York: Springer 1985

Krichever, I.M., Novikov, S.P.: Virasoro algebra, Riemann sirfaces and structure theory of soliton. Funct. Anal. Appl. 21, 46 (1987) (in Russian)

Krichever, I.M.: Methods of algebraic geometry in the theory of non-linear equations. Russ. Math. Surv. 32, 185 (1977)

Mulase, M.: Cohomological structure in soliton equations and jacobian varieties. J. Diff. Geom. 19, 403 (1984)

Mumford, D.: Tata lectures on theta, Vols. I, II. Boston: Birkhäuser 1983

Mumford, D. : An algebro-geometric construction of commuting operators and of solutions to the Toda lattice equation, Korteweg-de-Vries equation and related non-linear equations, Intern. Symp. on Algebraic Geometry, Kyoto, 1977, 115

Mumford, D.: Curves and Jacobians. Ann Arbor, MI: The University of Michigan Press 1975

Namikawa, Y.: On the canonical holomorphic map from the moduli space of stable curves to the Igusa monodial transform. Nagoya Math. J. 52, 197 (1973)

Peterson, D.H., Kac, V.: Infinite flag varieties and conjugacy theorems. Proc. Nat. Acad. Sci. USA, 80, 1778 (1983)

Polyakov, A.M.: Quantum geometry of bosonic strings. Phys. Lett. 103B, 207 (1981)

Sato, M.: Soliton equations as dynamical systems on an infinite dimensional Grassmann manifold. Res. Inst. Math. Sci., Kyoto Univ.-Kokyuroku 439, 30 (1981) 
[S.N.] Sato, M., Noumi, M.: Soliton equation and universal Grassmann manifold. Sophia University Kokyuroku in Math. 18 (1984) (in Japanese)

[S.S.] Sato, M., Sato, Y.: Solition equations as dynamical systems on infinite dimensional Grassmann manifold, Lecture Notes in Num. Appl. Anal. 5, 259 (1982), Nonlinear PDE in Applied Science. U.S.-Japan Seminar, Tokyo, 1982

[S.W.] Segal, G.B., Wilson, G. : Loop groups and equations of KdV type, Publ. Math. I.H.E.S. 61, 5 (1985)

[Sh.] Shiota, T.: Characterization of jacobian varieties in terms of soliton equations. Invent. Math. 333 (1986)

[Si.] Siegel, C.L.: Topics of complex function theory, Vols. I, II. New York: Wiley

[So.] Sonoda, H.: Calculation of a propagator on a Riemann surface LBL preprint, LBL-21877, The energy-momentum tensor on a Riemann surface. Nucl. Phys. B281, 546 (1987)

[T.K.1] Tsuchiya, A., Kanie, Y.: Fock space representation of the Virasoro algebraintertwining operators. Publ. R.I.M.S. Kyoto Univ. 259, 22 (1986)

[T.K.2] Tsuchiya, A., Kanie, Y.: Vertex operators on conformal field theory on $\mathbb{P}^{1}$ and monodromy representations of Braid groups, To appear in: Conformal field theory and solvable lattice model. Advanced Studies in Pure Mathematics, Kinokuniya. Lett. Math. Phys. 13, 303 (1987)

[V.] Vafa, C.: Operator formulation on Riemann surfaces. Phys. Lett. 190 B, 47 (1987)

[V.V.] Verlinde, E., Verlinde, H.: Chiral bosonization, determinants and string partition function. Nucl. Phys. B288, 357 (1987)

Communicated by H. Araki

Received July 27, 1987; in revised form November 16, 1987

Notes added in proof. After submission of this paper we were informed that several new papers had appeared which treat similar subjects as our paper: L. Alvarez-Gaumé, C. Comez and C. Reina: New methods in string theory, CERN preprint, CERN-TH 6775/87. E. Arbarello, C. De Concini, V. Kac, C. Procesi: Moduli spaces of curves and representation theory, Commun. Math. Phys., to appear. E. Witten: Quantum field theory, grassmannians, and algebraic curves. Commun. Math. Phys. (to appear). 